\title{
The Second Spectrum of Ruthenium (Ru II)
}

\author{
Allen G. Shenstone ${ }^{1}$ and William F. Meggers
}

\begin{abstract}
Wavelengths and estimated intensities of 2,227 spectral lines characteristic of singly ionized ruthenium atoms are presented. The wavelengths range from 1054.684 to $6371.29 \mathrm{~A}$. The Zeeman effect has been investigated for 488 lines ranging from 2323 to $4010 \mathrm{~A}$. Analysis of these basic data of $\mathrm{Ru}$ II has resulted in the classification of 1,633 lines as transitions between 68-even, and 140-odd energy levels. All but three of these levels have been grouped into designated spectral terms and assigned to electron configurations. The low-even terms arise from $4 d^{7}, 4 d^{6} 5 s$, and $4 d^{5} 5 s^{2}$. All the terms from the $4 d^{7}$ configuration have been found, and $4 d^{7} a^{4} \mathrm{~F}$ represents the ground state of Ru II. From two members of the $4 d^{6} n s^{6} \mathrm{D}$ series a limit, $135200 \mathrm{~cm}^{-1}$, has been calculated which indicates a principal ionization potential (I. P.) of 16.76 electron volts for $\mathrm{Ru}^{+}$ions
\end{abstract}

As far back as $1930[1]^{2}$ an analysis was undertaken of the first spark spectrum of ruthenium, element number 44. Although the fundamental multiplets were easily found, the complexity of the spectrum made further work unrewarding until more complete and accurate measurements could be made. In 1955 [2] Kessler and Meggers published new measurements, and in 1952 Meggers measured Zeeman effects on plates taken at the Massachusetts Institute of Technology with their large-field magnet and high-dispersion spectrographs. In addition, the spectrum was photographed on the Princeton vacuum instrument to provide wavelengths from $500 \mathrm{~A}$ to $2200 \mathrm{~A}$. This instrument uses a 30,000 line per inch grating of $2 \mathrm{~m}$ radius of curvature in a normal incidence mounting. All of the measurements of wavelength were made from spectrograms of the spark, a source which is not altogether satisfactory for first-spark spectra. For instance, Dobbie's [3] excellent analysis of FeII was made entirely from the polar lines in an are. Therefore, some further observations were made with such a source, and a considerable number of lines were found in the longer wavelength region which were not observed in the spark. All of the arc and spark photographs were made with buttons of pure compressed ruthenium powder. The metallic impurities were very faint and easily eliminated. In the vacuum region the gas and carbon lines, which are always present, were used as standards. In that region the differentiation of stages of ionization was made from an examination of the polarity of the lines on a number of plates. In some cases, it was even possible to distinguish lines due to the lowest levels from those due to levels only 20000 $\mathrm{cm}^{-1}$ higher. As usual, lines due to transitions from high even levels to intermediate odd levels are very wide and displaced from the position they occupy in an arc source.

Although the Zeeman effect on ruthenium lines was photographed and machine-measured at the Massachusetts Institute of Technology in 1940 only the results for $\mathrm{Ru}$ I were exploited and published

1 Present address: Palmer Physical Laboratory, Princeton University, Princeton, N. J.

${ }_{2}^{2}$ Figures in brackets indicate the literature references on page 378 .
[4]. In 1949 new Zeeman spectrograms of ruthenium were made at the Massachusetts Institute of Technology but the magnetic field intensity did not exceed 80,000 oersteds. Because some of the earlier spectrograms were made with higher field intensities which greatly enhanced RuII, the best of these $(Z-74)$ were loaned to the National Bureau of Standards where they were hand-measured and computed. A field intensity of 88,200 oersteds was derived from the magnetic splitting of impurity lines (Mg II 2795.53 and 2802.60 A, Cu I 3247.54 and $3273.96 \mathrm{~A}, \mathrm{Ag}$ I 3280.68 and $3382.89 \mathrm{~A}$, and Ca II 3933.66 and $3968.47 \mathrm{~A}$ ).

The $\mathrm{RuII}$ lines were easily distinguished from Ru I by comparing the total intensities of the Zeeman patterns with the intensities in the central comparison spectrum produced without the magnetic field: the $\mathrm{Ru}$ II lines were greatly enhanced by the magnetic field. In addition, the fully recorded and resolved patterns of RuII lines were recognized bv their types 4,5 , and 6 characteristic of even multiplicities according to Back and Landé [5]. Type 4 exhibits two symmetrically least-displaced $p$-components of equal intensity, and the two strongest $n$-components are also least displaced, when the level with the larger $J$ has the smaller $g$. Type 5 occurs when the larger $J$ has the larger $g$, in which case the two strongest $n$-components exhibit the maximum displacement. Type 6 is produced by levels with equal $J$ but unequal $g$, in which case the strongest $p$-components show the largest displacement, and the strongest $n$-components an average displacement. The observed $g$-factors given to 3 decimals in tables 1,2 , and 5 are the average of all calculations from resolved patterns, indicated in table 6 by $\Delta g$. Only the displacements of maximum intensities of unresolved patterns on the MIT spectrograms were measured, and the types noted. Then after such a line was classified it was often possible to calculate a $g$-factor for one level if the other one is known. In many type 6 patterns the over-all width $(w)$ of the unresolved $p$-components was measured so that $g$ values could be calculated for both energy levels when their $J$-values were fixed by the classification; in these cases the $\Delta g$ (in table 6) is inclosed in parentheses and the $g$-factors are given to two deci- 
mals. For a few levels, the $g$-values were calculated to two decimals from unresolved patterns on plates taken 30 years ago at Princeton, according to a procedure described by Shenstone and Blair [6].

As remarked above, the lowest multiplets were found many years ago; but even after the new wavelength measurements were made and Zeeman effects observed, it still proved very difficult to break into the higher groups of terms. This was surprising since in a spectrum of this kind, one expects many intersystem and interfamily lines. There are many such combinations but few of them are with the sextet system. In point of fact, the strongest interfamily combinations with that system are in the long wavelength region where the lines can be observed only at the poles of the arc. This was unfortunately discovered after the analysis was practically complete.

The break into the higher quartet terms was made through a search in the Schumann region for the differences occurring in the low ${ }^{4} \mathrm{~F}$ term. From such differences the usual square array was built up, though the work was not made any easier by the fact that great reliance was placed on a comparison with Fe II.
Only after a very arduous numerical analysis was it possible to assign term designations to the even levels and a reasonable identification of odd levels was impossible until the analysis was nearly complete. A warning must be given against too great reliance on the names chosen. The levels are so mixed in character that the most that can be hoped is that designations have been selected which correspond to the particular Russell-Saunders type that is the chief contributor to each level. The terms of $4 d^{7}$ and $4 d^{6}\left({ }^{5} \mathrm{D}\right) 5$ s are the most reliably named because they are reasonably pure. The remaining even levels are in general less pure the higher they are.

The names of the odd levels are considerably less reliable than those of the even levels. The levels were found chiefly by the numerical analysis mentioned above, assisted by rough predictions of position and intensity of combinations. Because the spectrum Te I, isoelectronic with $\mathrm{Ru} \mathrm{II}$, is only partially analyzed [7], the chief reliance had to be on the homologous spectrum Fe II [8] for predictions of position. Unfortunately, the correspondence is much rougher than was expected. For instance, the terms $a^{4} \mathrm{H}$ and

TABLE 1. Even levels of RuII

\begin{tabular}{|c|c|c|c|c|c|c|c|c|c|c|c|}
\hline Configuration & $\begin{array}{l}\text { Desig- } \\
\text { nation }\end{array}$ & $J$ & Level & $g(\mathrm{obs})$ & $g$ (Landé) & Configuration & $\begin{array}{l}\text { Desig- } \\
\text { nation }\end{array}$ & $J$ & Level & $g(\mathrm{obs})$ & $g$ (Landé) \\
\hline $\begin{array}{l}4 d^{7} \\
4 d^{7} \\
4 d^{7} \\
4 d^{7} \\
4 d^{7}\end{array}$ & $\begin{array}{l}a a^{4} \mathrm{~F} \\
a^{4} \mathrm{~F} \\
a^{4} \mathrm{~F} \\
a^{4} \mathrm{~F} \\
a^{4} \mathrm{~F}\end{array}$ & $\begin{array}{l}4^{1 / 2} \\
3^{1 / 2} \\
2^{1 / 2} \\
1^{1 / 2} \\
2^{1 / 2}\end{array}$ & $\begin{array}{r}0.0 \\
\text { 1523. } 1 \\
2493.9 \\
\text { 3104. } 2 \\
\text { 8256. }\end{array}$ & 1.57 & $\begin{array}{l}\text { 1. } 333 \\
\text { 1. } 238 \\
\text { 1. } 029 \\
0.419 \\
\text { 1. } 600\end{array}$ & $\begin{array}{l}4 d^{6}\left({ }^{3} \mathrm{G}\right) 5 s \\
4 d^{6}\left({ }^{3} \mathrm{P}\right) 5 s \\
4 d^{6}\left({ }^{3} \mathrm{G}\right) 5 s \\
4 d^{6}\left(^{3} \mathrm{G}\right) 5 s \\
4 d^{6}\left({ }^{3} \mathrm{G}\right) 5 s\end{array}$ & $\begin{array}{ll}a & { }^{4} \mathrm{G} \\
b & { }^{4} \mathrm{P} \\
a & { }^{4} \mathrm{G} \\
a & { }^{4} \mathrm{G} \\
a^{4} & { }^{4} \mathrm{G}\end{array}$ & $\begin{array}{l}5^{1 / 2} / 2 \\
1^{1 / 2} \\
4^{1 / 2} \\
2^{1} / 2 \\
3^{1 / 2}\end{array}$ & $\begin{array}{l}29018.6 \\
29091.0 \\
30099.6 \\
30293.3 \\
30439.5\end{array}$ & $\begin{array}{l}1.232 \\
1.646 \\
1.167 \\
0.651 \\
1.021\end{array}$ & $\begin{array}{l}\text { 1. } 273 \\
\text { 1. } 733 \\
1.172 \\
0.571 \\
0.984\end{array}$ \\
\hline $\begin{array}{l}4 d^{7} \\
4 d^{6}\left({ }^{5} \mathrm{D}\right) 5 s \\
4 d^{7} \\
4 d^{6}\left({ }^{5} \mathrm{D}\right) 5 s \\
4 d^{6}\left({ }^{5} \mathrm{D}\right) 5 s\end{array}$ & $\begin{array}{ll}a & { }^{4} \mathrm{P} \\
a & { }^{6} \mathrm{D} \\
a & 4 \\
a & \mathrm{P} \\
a & 6 \mathrm{D} \\
a^{6} & { }^{6} \mathrm{D}\end{array}$ & $\begin{array}{l}1^{1 / 2} / 2 \\
4^{1 / 2} \\
0^{1 / 2} \\
3^{1 / 2} \\
2^{1} \frac{1}{2}\end{array}$ & $\begin{array}{r}8477.7 \\
9151.4 \\
9373.9 \\
10150.4 \\
10851.7\end{array}$ & $\begin{array}{l}1.68 \\
1.53 \\
2.60 \\
1.576 \\
1.641\end{array}$ & $\begin{array}{l}\text { 1. } 733 \\
\text { 1. } 556 \\
\text { 2. } 667 \\
\text { 1. } 587 \\
\text { 1. } 657\end{array}$ & $\begin{array}{l}4 d^{6}\left({ }^{3} \mathrm{P}\right) 5 s \\
4 d^{6}\left({ }^{3} \mathrm{H}\right) 5 s \\
4 d^{3}\left({ }^{3} \mathrm{H}\right) 5 s \\
4 d^{6}\left({ }^{3} \mathrm{D}\right) 5 s \\
4 d^{6}\left({ }^{3} \mathrm{D}\right) 5 s\end{array}$ & $\begin{array}{ll}b & { }^{4} \mathrm{P} \\
b & 2 \\
b & \mathrm{H} \\
b & { }^{2} \mathrm{H} \\
b & { }^{4} \mathrm{D} \\
b & { }^{4} \mathrm{D}\end{array}$ & $\begin{array}{l}0^{1 / 2} \\
5^{1 / 2} \\
4^{1 / 2} \\
1^{1 / 2} \\
0^{1 / 2}\end{array}$ & $\begin{array}{l}30489.3 \\
32623.0 \\
32686.7 \\
32888.4 \\
32960.8\end{array}$ & $\begin{array}{l}2.541 \\
1.065 \\
0.955 \\
1.200 \\
0.138\end{array}$ & $\begin{array}{l}\text { 2. } 667 \\
\text { 1. } 091 \\
\text { 0. } 909 \\
\text { 1. } 200 \\
0.00\end{array}$ \\
\hline $\begin{array}{l}4 d^{7} \\
4 d^{6}\left({ }^{5} \mathrm{D}\right) 5 s \\
4 d^{6}\left({ }^{5} \mathrm{D}\right) 5 s \\
4 d^{7} \\
4 d^{7}\end{array}$ & $\begin{array}{l}a{ }^{2} \mathrm{G} \\
a^{6} \mathrm{D} \\
a^{6}{ }^{6} \mathrm{D} \\
a^{2}{ }^{2} \mathrm{G} \\
a^{2} \mathrm{P}\end{array}$ & $\begin{array}{l}4^{1 / 2} \\
11 \frac{2}{2} \\
01 \frac{2}{31 / 2} \\
11 \frac{1}{2}\end{array}$ & $\begin{array}{l}10860.9 \\
11303.8 \\
11604.0 \\
12293.4 \\
12956.6\end{array}$ & $\begin{array}{l}\text { 1. } 843 \\
\text { 3. } 271 \\
\end{array}$ & $\begin{array}{l}\text { 1. } 111 \\
\text { 1. } 867 \\
\text { 3. } 333 \\
0.889 \\
\text { 1. } 333\end{array}$ & $\begin{array}{l}4 d^{6}\left({ }^{3} \mathrm{D}\right) 5 s \\
4 d^{6}\left({ }^{3} \mathrm{D}\right) 5 s \\
4 d^{6}\left({ }^{3} \mathrm{P}\right) 5 s \\
4 d^{6}\left({ }^{3} \mathrm{~F}\right) 5 s \\
4 d^{7}\end{array}$ & $\begin{array}{ll}b & { }^{4} \mathrm{D} \\
b & { }^{4} \mathrm{D} \\
b & 2 \mathrm{P} \\
b & { }^{2} \mathrm{~F} \\
b & { }^{2} \mathrm{D}\end{array}$ & $\begin{array}{l}2^{1 / 2} \\
3^{1 / 2} \\
1^{1 / 2} \\
3^{1 / 2} \\
1^{1 / 2}\end{array}$ & $\begin{array}{l}33018.8 \\
33332.7 \\
33734.8 \\
34038.3 \\
34793.1\end{array}$ & $\begin{array}{l}\text { 1. } 338 \\
\text { 1. } 397 \\
\text { 1. } 185 \\
\text { 1. } 083 \\
0.965\end{array}$ & $\begin{array}{l}\text { 1. } 371 \\
\text { 1. } 429 \\
\text { 1. } 333 \\
\text { 1. } 143 \\
\text { 0. } 800\end{array}$ \\
\hline $\begin{array}{l}4 d^{7} \\
4 d^{7} \\
4 d^{7} \\
4 d^{7} \\
4 d^{7}\end{array}$ & $\begin{array}{ll}a^{2} & 2 \mathrm{D} \\
a^{2} & \mathrm{H} \\
a^{2} & 2 \mathrm{P} \\
a^{2} & 2 \mathrm{H} \\
a^{2} & { }^{2} \mathrm{D}\end{array}$ & $\begin{array}{l}21 / 2 \\
5^{1 / 2} \\
0^{1 / 2} \\
4^{1 / 2} \\
1^{1 / 2}\end{array}$ & $\begin{array}{l}14581.2 \\
14663.4 \\
14799.5 \\
16125.0 \\
17017.6\end{array}$ & $\ldots$ & $\begin{array}{l}\text { 1. } 200 \\
1.091 \\
0.667 \\
0.909 \\
0.800\end{array}$ & $\begin{array}{l}4 d^{7} \\
4 d^{6}\left({ }^{3} \mathrm{~F}\right) 5 s \\
4 d^{5} 5 s^{2} \\
4 d^{6}\left({ }^{1} \mathrm{I}\right) 5 s \\
4 d^{6}\left({ }^{3} \mathrm{G}\right) 5 s\end{array}$ & $\begin{array}{ll}b & { }^{2} \mathrm{D} \\
b & 2 \\
a & 6 \mathrm{~F} \\
a^{2} & { }^{2} \mathrm{I} \\
b^{2} & { }^{2} \mathrm{G}\end{array}$ & $\begin{array}{l}2^{1 / 2} / 2 \\
2^{1 / 2} \\
2^{1 / 2} \\
6^{1 / 2} \\
4^{1 / 2}\end{array}$ & $\begin{array}{l}34829.3 \\
35298.4 \\
35857.6 \\
35939.6 \\
36016.0\end{array}$ & $\begin{array}{l}\text { 1. } 102 \\
0.945 \\
\text { 1. } 06 \\
\text { 1. } 067\end{array}$ & $\begin{array}{l}\text { 1. } 200 \\
0.857 \\
\text { 2. } 000 \\
\text { 1. } 077 \\
\text { 1. } 111\end{array}$ \\
\hline 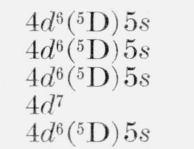 & $\begin{array}{ll}a & 4 \\
a & \mathrm{D} \\
{ }^{4} \mathrm{D} \\
a & { }^{4} \mathrm{D} \\
a^{2} & \mathrm{~F} \\
a^{4} \mathrm{D}\end{array}$ & $\begin{array}{l}31 / 2 \\
21 / 2 \\
11 / 2 \\
21 / 2 \\
01 / 2\end{array}$ & $\begin{array}{l}19378.7 \\
20515.0 \\
21246.4 \\
21557.8 \\
21645.6\end{array}$ & $\begin{array}{l}\text { 1. } 402 \\
\text { 1. } 337 \\
\text { 1. } 188 \\
0.863 \\
0.015\end{array}$ & $\begin{array}{l}\text { 1. } 429 \\
\text { 1. } 371 \\
\text { 1. } 200 \\
0.857 \\
0.000\end{array}$ & $\begin{array}{l}4 d^{6}\left({ }^{3} \mathrm{P}\right) 5 s \\
4 d^{6}\left({ }^{1} \mathrm{I}\right) 5 s \\
4 d^{3}\left({ }^{3} \mathrm{G}\right) 5 s \\
4 d^{6}\left({ }^{1} \mathrm{G}\right) 5 s \\
4 d^{6}\left({ }^{1} \mathrm{G}\right) 5 s\end{array}$ & $\begin{array}{ll}b & { }^{2} \mathrm{P} \\
a & 2 \\
b & \mathrm{I} \\
b & { }^{2} \mathrm{G} \\
c & { }^{2} \mathrm{G} \\
c & { }^{2} \mathrm{G}\end{array}$ & $\begin{array}{l}0^{1 / 2} \\
5^{1 / 2} \\
3^{1 / 2} \\
4^{1 / 2} \\
3^{1 / 2}\end{array}$ & $\begin{array}{l}36095.0 \\
36229.7 \\
36515.8 \\
37433.0 \\
37981.5\end{array}$ & $\begin{array}{l}0.875 \\
0.93 \\
0.885 \\
1.078 \\
0.942\end{array}$ & $\begin{array}{l}0.667 \\
0.923 \\
0.889 \\
1.111 \\
0.889\end{array}$ \\
\hline $\begin{array}{l}4 d^{7} \\
4 d^{6}\left({ }^{3} \mathrm{H}\right) 5 s \\
4 d^{6}\left({ }^{3} \mathrm{H}\right) 5 s \\
4 d^{6}\left({ }^{3} \mathrm{H}\right) 5 s \\
4 d^{6}\left({ }^{3} \mathrm{H}\right) 5 s\end{array}$ & $\begin{array}{l}a{ }^{2} \mathrm{~F} \\
a \\
{ }^{4} \mathrm{H} \\
{ }^{4} \mathrm{H} \\
a \\
a^{4} \mathrm{H} \\
{ }^{4} \mathrm{H}\end{array}$ & $\begin{array}{l}31 / 2 \\
6^{1 / 2} \\
5^{1 / 2} \\
4^{1 / 2} \\
3^{1 / 2}\end{array}$ & $\begin{array}{l}22289.0 \\
25952.2 \\
26109.4 \\
26118.4 \\
26468.0\end{array}$ & $\begin{array}{l}\text { 1. } 138 \\
\text { 1. } 235 \\
\text { 1. } 16 \\
\text { 1. } 08 \\
0.759\end{array}$ & $\begin{array}{l}1.143 \\
1.231 \\
1.133 \\
0.970 \\
0.667\end{array}$ & $\begin{array}{l}4 d^{6}\left({ }^{3} \mathrm{D}\right) 5 s \\
4 d^{6}\left({ }^{3} \mathrm{D}\right) 5 s \\
4 d^{6}\left({ }^{1} \mathrm{~S}\right) 5 s \\
4 d^{6}\left({ }^{1} \mathrm{D}\right) 5 s \\
4 d^{6}\left({ }^{1} \mathrm{D}\right) 5 s\end{array}$ & 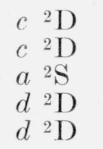 & $\begin{array}{l}2^{1 / 2} \\
1^{1 / 2} \\
0^{1 / 2} \\
1^{1 / 2} \\
2^{1 / 2}\end{array}$ & $\begin{array}{l}38981.8 \\
39711.9 \\
40226.2 \\
42750.1 \\
43609.9\end{array}$ & $\begin{array}{l}1.18 \\
0.797 \\
1.800 \\
0.803 \\
1.216\end{array}$ & $\begin{array}{l}\text { 1. } 200 \\
\text { 0. } 800 \\
\text { 2. } 000 \\
0.800 \\
\text { 1. } 200\end{array}$ \\
\hline $\begin{array}{l}4 d^{6}\left({ }^{3} \mathrm{P}\right) 5 s \\
4 d^{6}\left(^{3} \mathrm{~F}\right) 5 s \\
4 d^{6}\left({ }^{3} \mathrm{~F}\right) 5 s \\
4 d^{6}\left({ }^{3} \mathrm{~F}\right) 5 s \\
4 d^{6}\left({ }^{3} \mathrm{~F}\right) 5 s\end{array}$ & $\begin{array}{ll}b & { }^{4} \mathrm{P} \\
b & { }^{4} \mathrm{~F} \\
b & { }^{4} \mathrm{~F} \\
b & { }^{4} \mathrm{~F} \\
b & { }^{4} \mathrm{~F}\end{array}$ & $\begin{array}{l}21 / 2 \\
41 / 2 \\
31 / 2 \\
21 / 2 \\
11 / 2\end{array}$ & $\begin{array}{l}26911.4 \\
27544.6 \\
27948.8 \\
28138.8 \\
28495.3\end{array}$ & $\begin{array}{l}\text { 1. } 578 \\
\text { 1. } 200 \\
\text { 1. } 091 \\
0.941 \\
0.420\end{array}$ & $\begin{array}{l}\text { 1. } 600 \\
\text { 1. } 333 \\
\text { 1. } 238 \\
1.029 \\
0.419\end{array}$ & $\begin{array}{l}4 d^{6}\left({ }^{5} \mathrm{D}\right) 6 s \\
4 d^{6}\left({ }^{5} \mathrm{D}\right) 6 s \\
4 d^{6}\left({ }^{5} \mathrm{D}\right) 6 s\end{array}$ & $\begin{array}{ll}e & { }^{6} \mathrm{D} \\
e & { }^{4} \mathrm{D} \\
e & { }^{4} \mathrm{D}\end{array}$ & $\begin{array}{l}4^{1 / 2} \\
3^{1 / 2} \\
2^{1 / 2}\end{array}$ & $\begin{array}{l}84510.9 \\
86440.4 \\
87523.4\end{array}$ & $\ldots$ & $\begin{array}{l}\text { 1. } 556 \\
\text { 1. } 429 \\
\text { 1. } 371\end{array}$ \\
\hline
\end{tabular}


TABLE 2. Odd levels of Ru II

\begin{tabular}{|c|c|c|c|c|c|c|c|c|c|c|c|}
\hline Configuration & $\begin{array}{l}\text { Desig- } \\
\text { nation }\end{array}$ & $J$ & Level & $g$ (obs) & $g$ (Landé) & Configuration & $\begin{array}{l}\text { Desig- } \\
\text { nation }\end{array}$ & $J$ & Level & $g(\mathrm{obs})$ & $g$ (Landé) \\
\hline $\begin{array}{l}4 d^{6}\left({ }^{5} \mathrm{D}\right) 5 p \\
4 d^{6}\left({ }^{5} \mathrm{D}\right) 5 p \\
4 d^{6}\left({ }^{5} \mathrm{D}\right) 5 p \\
4 d^{6}\left({ }^{5} \mathrm{D}\right) 5 p \\
4 d^{6}\left({ }^{5} \mathrm{D}\right) 5 p\end{array}$ & $\begin{array}{ll}z & { }^{6} \mathrm{D}^{\circ} \\
z & { }^{6} \mathrm{D}^{\circ} \\
z & { }^{6} \mathrm{D}^{\circ} \\
z & { }^{6} \mathrm{D}^{\circ} \\
z & { }^{6} \mathrm{D}^{\circ}\end{array}$ & $\begin{array}{l}4^{1 / 2} \\
3^{1 / 2} \\
2^{1 / 2} \\
11 / 2 \\
01 / 2\end{array}$ & $\begin{array}{l}46471.0 \\
46711.5 \\
47285.1 \\
47708.6 \\
47983.8\end{array}$ & $\begin{array}{l}\text { 1. } 530 \\
\text { 1. } 569 \\
\text { 1. } 634 \\
\text { 1. } 840 \\
\text { 3. } 280\end{array}$ & $\begin{array}{l}\text { 1. } 556 \\
\text { 1. } 587 \\
1.657 \\
1.867 \\
3.333\end{array}$ & $\begin{array}{l}4 d^{6}\left({ }^{3} \mathrm{G}\right) 5 p \\
4 d^{6}\left({ }^{3} \mathrm{~F}\right) 5 p \\
4 d^{6}\left({ }^{3} \mathrm{~F}\right) 5 p \\
4 d^{6}\left({ }^{3} \mathrm{H}\right) 5 p \\
4 d^{6}\left({ }^{3} \mathrm{~F}\right) 5 p\end{array}$ & $\begin{array}{ll}x & { }^{4} \mathrm{~F}^{\circ} \\
y & { }^{4} \mathrm{G}^{\circ} \\
x & { }^{4} \mathrm{D}^{\circ} \\
z & { }^{2} \mathrm{H}^{\circ} \\
x & { }^{4} \mathrm{D}^{\circ}\end{array}$ & $\begin{array}{l}4^{1 / 2} \\
2^{1 / 2} \\
0^{1 / 2} \\
4^{1 / 2} \\
3^{1 / 2}\end{array}$ & $\begin{array}{l}67900.9 \\
67946.6 \\
68096.9 \\
68120.3 \\
68229.3\end{array}$ & $\begin{array}{l}\text { 1. } 19 \\
0.689 \\
0.385 \\
1.015 \\
0.955\end{array}$ & $\begin{array}{l}\text { 1. } 333 \\
0.571 \\
0.000 \\
0.909 \\
1.429\end{array}$ \\
\hline $\begin{array}{l}4 d^{6}\left({ }^{5} \mathrm{D}\right) 5 p \\
4 d^{6}\left({ }^{5} \mathrm{D}\right) 5 p \\
4 d^{6}\left({ }^{5} \mathrm{D}\right) 5 p \\
4 d^{6}\left({ }^{5} \mathrm{D}\right) 5 p \\
4 d^{6}\left({ }^{5} \mathrm{D}\right) 5 p\end{array}$ & $\begin{array}{ll}z & { }^{6} \mathrm{~F}^{\circ} \\
z & 6 \mathrm{~F}^{\circ} \\
z & { }^{6} \mathrm{~F}^{\circ} \\
z & { }^{6} \mathrm{~F}^{\circ} \\
z^{6} & \mathrm{~F}^{\circ}\end{array}$ & $\begin{array}{l}5^{1 / 2} \\
4^{1 / 2} \\
3^{1 / 2} \\
2^{1 / 2} \\
11 / 2\end{array}$ & $\begin{array}{l}50758.3 \\
50845.3 \\
50862.5 \\
51179.7 \\
5131 \% .0\end{array}$ & $\begin{array}{l}\text { 1. } 44 \\
\text { 1. } 42 \\
\text { 1. } 457 \\
\text { 1. } 312 \\
\text { 1. } 058\end{array}$ & $\begin{array}{l}\text { 1. } 455 \\
\text { 1. } 434 \\
\text { 1. } 397 \\
\text { 1. } 314 \\
\text { 1. } 067\end{array}$ & $\begin{array}{l}4 d^{6}\left({ }^{3} \mathrm{~F}\right) 5 p \\
4 d^{6}\left({ }^{3} \mathrm{G}\right) 5 p \\
4 d^{6}\left({ }^{3} \mathrm{P}\right) 5 p \\
4 d^{6}\left({ }^{3} \mathrm{P}\right) 5 p \\
4 d^{6}\left({ }^{3} \mathrm{G}\right) 5 p\end{array}$ & $\begin{array}{ll}y & { }^{2} \mathrm{G}^{\circ} \\
x & 4 \mathrm{G}^{\circ} \\
z & 2 \mathrm{P}^{\circ} \\
y & { }^{4} \mathrm{D}^{\circ} \\
x & { }^{4} \mathrm{G}^{\circ}\end{array}$ & $\begin{array}{l}3^{1 / 2} \\
5^{1 / 2} \\
11 / 2 \\
2^{1 / 2} \\
4^{1 / 2}\end{array}$ & $\begin{array}{l}68265.6 \\
68328.3 \\
684.45 .8 \\
68773.9 \\
68906.5\end{array}$ & $\begin{array}{l}\text { 1. } 028 \\
\text { 1. } 152 \\
1.160 \\
1.308 \\
1.17\end{array}$ & $\begin{array}{l}\text { 0. } 889 \\
\text { 1. } 273 \\
\text { 1. } 333 \\
\text { 1. } 371 \\
\text { 1. } 172\end{array}$ \\
\hline $\begin{array}{l}4 d^{6}\left({ }^{5} \mathrm{D}\right) 5 p \\
4 d^{6}\left({ }^{5} \mathrm{D}\right) 5 p \\
4 d^{6}\left({ }^{5} \mathrm{D}\right) 5 p \\
4 d^{6}\left({ }^{5} \mathrm{D}\right) 5 p \\
4 d^{6}\left({ }^{5} \mathrm{D}\right) 5 p\end{array}$ & $\begin{array}{ll}z & { }^{6} \mathrm{~F}^{\circ} \\
z & { }^{6} \mathrm{P}^{\circ} \\
z & { }^{6} \mathrm{P}^{\circ} \\
z & { }^{4} \mathrm{~F}^{\circ} \\
z & { }^{4} \mathrm{D}^{\circ}\end{array}$ & $\begin{array}{l}01 / 2 \\
31 / 2 \\
21 / 2 \\
41 / 2 \\
31 / 2\end{array}$ & $\begin{array}{l}51380.1 \\
51548.8 \\
52820.3 \\
52964.4 \\
53317.1\end{array}$ & $\begin{array}{l}-0.62 \\
1.580 \\
1.835 \\
1.346 \\
1.391\end{array}$ & $\begin{array}{r}-0.667 \\
1.714 \\
1.886 \\
1.333 \\
1.429\end{array}$ & $\begin{array}{l}4 d^{6}\left({ }^{3} \mathrm{G}\right) 5 p \\
4 d^{6}\left({ }^{3} \mathrm{~F}\right) 5 p \\
4 d^{6}\left({ }^{3} \mathrm{G}\right) 5 p \\
4 d^{6}\left({ }^{3} \mathrm{G}\right) 5 p \\
4 d^{6}\left({ }^{3} \mathrm{P}\right) 5 p\end{array}$ & 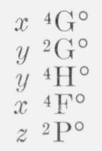 & $\begin{array}{l}21 / 2 \\
4^{1 / 2} \\
6^{1 / 2} \\
3^{1 / 2} \\
0^{1 / 2} / 2\end{array}$ & $\begin{array}{l}69054.8 \\
69180.8 \\
69311.1 \\
69404.1 \\
69439.5\end{array}$ & $\begin{array}{l}0.706 \\
1.058 \\
1.180 \\
1.059 \\
0.829\end{array}$ & $\begin{array}{l}0.571 \\
1.111 \\
1.231 \\
1.238 \\
0.667\end{array}$ \\
\hline $\begin{array}{l}4 d^{6}\left({ }^{5} \mathrm{D}\right) 5 p \\
4 d^{6}(5 \mathrm{D}) 5 p \\
\left.4 d^{6}{ }^{5} \mathrm{D}\right) 5 p \\
4 d^{6}\left({ }^{5} \mathrm{D}\right) 5 p \\
4 d^{6}\left({ }^{5} \mathrm{D}\right) 5 p\end{array}$ & $\begin{array}{ll}z & { }^{6} \mathrm{P}^{\circ} \\
z & 4 \\
{ }^{4} \mathrm{D}^{\circ} \\
z & { }^{4} \mathrm{~F}^{\circ} \\
z & { }^{4} \mathrm{D}^{\circ} \\
z & { }^{4} \mathrm{~F}^{\circ}\end{array}$ & $\begin{array}{l}11 / 2 \\
21 / 2 \\
31 / 2 \\
11 / 2 \\
21 / 2\end{array}$ & $\begin{array}{l}53685.2 \\
54065.4 \\
54225.5 \\
54663.4 \\
54794.3\end{array}$ & $\begin{array}{l}\text { 2. } 365 \\
\text { 1. } 348 \\
\text { 1. } 274 \\
\text { 1. } 145 \\
\text { 1. } 077\end{array}$ & $\begin{array}{l}\text { 2. } 400 \\
\text { 1. } 371 \\
\text { 1. } 238 \\
\text { 1. } 200 \\
\text { 1. } 029\end{array}$ & $\begin{array}{l}4 d^{6}\left({ }^{3} \mathrm{G}\right) 5 p \\
4 d^{6}\left({ }^{3} \mathrm{G}\right) 5 p \\
4 d^{6}\left({ }^{3} \mathrm{G}\right) 5 p \\
4 d^{6}\left({ }^{3} \mathrm{G}\right) 5 p \\
4 d^{6}\left({ }^{3} \mathrm{G}\right) 5 p\end{array}$ & $\begin{array}{ll}x & { }^{4} \mathrm{G}^{\circ} \\
y & { }^{4} \mathrm{H}^{\circ} \\
x & { }^{4} \mathrm{~F}^{\circ} \\
x & { }^{4} \mathrm{~F}^{\circ} \\
y & { }^{4} \mathrm{H}^{\circ}\end{array}$ & $\begin{array}{l}31 / 2 \\
4^{1 / 2} \\
11 / 2 \\
21 / 2 \\
5^{1 / 2}\end{array}$ & $\begin{array}{l}69552.5 \\
69612.9 \\
69619.9 \\
69646.4 \\
69646.9\end{array}$ & $\begin{array}{l}1.00 \\
0.99 \\
0.648 \\
0.99 \\
1.108\end{array}$ & $\begin{array}{l}0.984 \\
0.970 \\
0.400 \\
1.029 \\
1.133\end{array}$ \\
\hline $\begin{array}{l}4 d^{6}\left({ }^{5} \mathrm{D}\right) 5 p \\
4 d^{6}\left({ }^{5} \mathrm{D}\right) 5 p \\
4 d^{6}\left({ }^{5} \mathrm{D}\right) 5 p \\
4 d^{6}\left({ }^{5} \mathrm{D}\right) 5 p \\
4 d^{6}\left({ }^{5} \mathrm{D}\right) 5 p\end{array}$ & 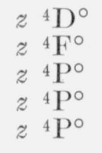 & $\begin{array}{l}01 / 2 \\
11 / 2 \\
21 / 2 \\
11 / 2 \\
01 / 2\end{array}$ & $\begin{array}{l}54981.5 \\
55224.2 \\
55695.2 \\
56664.9 \\
57263.8\end{array}$ & $\begin{array}{l}0.011 \\
0.468 \\
\text { 1. } 570 \\
\text { 1. } 708 \\
\text { 2. } 648\end{array}$ & $\begin{array}{l}0.000 \\
0.400 \\
1.600 \\
1.733 \\
\text { 2. } 667\end{array}$ & $\begin{array}{l}4 d^{6}\left({ }^{3} \mathrm{G}\right) 5 p \\
4 d^{6}\left({ }^{3} \mathrm{~F}\right) 5 p \\
4 d^{6}\left({ }^{3} \mathrm{~F}\right) 5 p \\
4 d^{6}\left({ }^{3} \mathrm{P}\right) 4 p\end{array}$ & 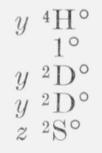 & $\begin{array}{l}31 / 2 \\
11 / 2 \\
21 / 2 \\
11 / 2 \\
01 / 2\end{array}$ & $\begin{array}{l}69655.5 \\
70150.8 \\
70571.9 \\
70779.6 \\
71036.3\end{array}$ & $\begin{array}{l}0.797 \\
1.209 \\
1.157 \\
0.744 \\
2.046\end{array}$ & $\begin{array}{l}0.667 \\
\text { 1. } 200 \\
\text { 0. } 800 \\
\text { 2. } 00\end{array}$ \\
\hline $\begin{array}{l}4 d^{6}\left({ }^{3} \mathrm{P}\right) 5 p \\
\left.4 d^{6}{ }^{(3} \mathrm{H}\right) 5 p \\
4 d^{6}\left({ }^{3} \mathrm{H}\right) 5 p \\
\left.4 d^{6}{ }^{3} \mathrm{H}\right) 5 p \\
4 d^{6}\left({ }^{3} \mathrm{H}\right) 5 p\end{array}$ & $\begin{array}{ll}z & { }^{4} \mathrm{~S}^{\circ} \\
z & { }^{4} \mathrm{H}^{\circ} \\
z & \mathrm{H}^{\circ} \mathrm{H}^{\circ} \\
z & { }^{4} \mathrm{H}^{\circ} \\
z & { }^{4} \mathrm{G}^{\circ}\end{array}$ & $\begin{array}{l}11 / 2 \\
31 / 2 \\
41 / 2 \\
51 / 2 \\
51 / 2\end{array}$ & $\begin{array}{l}62446.1 \\
62526.6 \\
62906.8 \\
6330 \% .7 \\
63518.5\end{array}$ & $\begin{array}{l}\text { 1. } 798 \\
0.961 \\
\text { 1. } 024 \\
\text { 1. } 068 \\
\text { 1. } 24\end{array}$ & $\begin{array}{l}\text { 2. } 000 \\
0.667 \\
0.970 \\
1.133 \\
1.273\end{array}$ & $\begin{array}{l}4 d^{6}\left({ }^{3} \mathrm{D}\right) 5 p \\
4 d^{6}\left({ }^{3} \mathrm{G}\right) 5 p \\
4 d^{6}\left({ }^{3} \mathrm{G}\right) 5 p \\
4 d^{6}\left({ }^{1} \mathrm{I}\right) 5 p \\
4 d^{6}\left({ }^{3} \mathrm{D}\right) 5 p\end{array}$ & 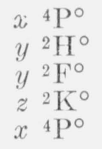 & $\begin{array}{l}2^{1 / 2} \\
5^{1 / 2} \\
2^{1 / 2} \\
6^{1 / 2} \\
1^{1 / 2} / 2\end{array}$ & $\begin{array}{l}\gamma_{1114.8} \\
y_{1202.9} \\
y_{1598 .} \\
y_{1798.3} \\
y_{1866.1}\end{array}$ & $\begin{array}{l}\text { 1. } 247 \\
\text { 1. } 32 \\
\text { 1. } 193 \\
0.91 \\
1.56\end{array}$ & $\begin{array}{l}\text { 1. } 600 \\
1.091 \\
0.857 \\
0.933 \\
1.733\end{array}$ \\
\hline $\begin{array}{l}4 d^{6}\left({ }^{3} \mathrm{H}\right) 5 p \\
4 d^{6}\left({ }^{3} \mathrm{P}\right) 5 p \\
4 d^{6}\left({ }^{3} \mathrm{H}\right) 5 p \\
4 d^{6}\left({ }^{3} \mathrm{H}\right) 5 p \\
4 d^{6}\left({ }^{3} \mathrm{H}\right) 5 p\end{array}$ & $\begin{array}{ll}z & { }^{4} \mathrm{H}^{\circ} \\
y & { }^{4} \mathrm{P}^{\circ} \\
z & 4 \mathrm{G}^{\circ} \\
z & { }^{4} \mathrm{G}^{\circ} \\
z & { }^{4} \mathrm{G}^{\circ}\end{array}$ & $\begin{array}{l}61 / 2 \\
2^{1 / 2} \\
4^{1} / 2 \\
31 / 2 \\
21 / 2\end{array}$ & $\begin{array}{l}63588.2 \\
6379 \% .6 \\
63851.3 \\
63941.2 \\
64025.3\end{array}$ & $\begin{array}{l}\text { 1. } 14 \\
\text { 1. } 295 \\
\text { 1. } 136 \\
\text { 0. } 978 \\
0.646\end{array}$ & $\begin{array}{l}\text { 1. } 231 \\
1.600 \\
1.172 \\
0.984 \\
0.571\end{array}$ & $\begin{array}{l}4 d^{6}\left({ }^{3} \mathrm{G}\right) 5 p \\
4 d^{6}\left({ }^{3} \mathrm{G}\right) 5 p \\
4 d^{6}\left({ }^{3} \mathrm{D}\right) 5 p \\
4 d^{6}\left({ }^{3} \mathrm{D}\right) 5 p \\
4 d^{5} 5 s\left({ }^{7} \mathrm{~S}\right) 5 p\end{array}$ & 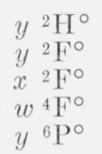 & $\begin{array}{l}4^{1 / 2} \\
31 / 2 \\
31 / 2 \\
11 / 2 \\
11 / 2\end{array}$ & $\begin{array}{l}71926.4 \\
72010.3 \\
72248.6 \\
72523.5 \\
72760.7\end{array}$ & $\begin{array}{l}0.96 \\
\text { 1. } 121 \\
\text { 1. } 14 \\
0.490 \\
\text { 2. } 49\end{array}$ & $\begin{array}{l}\text { 0. } 909 \\
\text { 1. } 143 \\
\text { 1. } 143 \\
\text { 0. } 400 \\
\text { 2. } 400\end{array}$ \\
\hline $\begin{array}{l}4 d^{6}\left({ }^{3} \mathrm{H}\right) 5 p \\
4 d^{6}\left({ }^{3} \mathrm{~F}\right) 5 p \\
4 d^{6}\left({ }^{3} \mathrm{~F}\right) 5 p \\
4 d^{6}\left({ }^{3} \mathrm{~F}\right) 5 p \\
4 d^{6}\left({ }^{3} \mathrm{H}\right) 5 p\end{array}$ & $\begin{array}{ll}z & { }^{4} \mathrm{I}^{\circ} \\
y & 4 \mathrm{H}^{\circ} \\
y & { }^{4} \mathrm{~F}^{\circ} \\
y & { }^{4} \mathrm{~F}^{\circ} \\
z & 2 \mathrm{G}^{\circ}\end{array}$ & $\begin{array}{l}41 / 2 \\
21 / 2 \\
31 / 2 \\
11 / 2 \\
31 / 2\end{array}$ & $\begin{array}{l}64385.3 \\
64463.8 \\
64748.8 \\
64921.6 \\
65212.9\end{array}$ & $\begin{array}{l}.959 \\
\text { 1. } 022 \\
\text { 1. } 045 \\
0.428 \\
\text { 1. } 032\end{array}$ & $\begin{array}{l}.727 \\
\text { 1. } 029 \\
\text { 1. } 238 \\
0.400 \\
0.889\end{array}$ & $\begin{array}{l}4 d^{6}\left({ }^{(1}\right) 5 p \\
4 d^{5} 5 s\left({ }^{7} \mathrm{~S}\right) 5 p \\
4 d^{5}\left({ }^{3} \mathrm{D}\right) 5 p \\
4 d^{5}\left({ }^{3} \mathrm{D}\right) 5 p\end{array}$ & $\begin{array}{ll} & 2^{\circ} \\
z & { }^{2} \mathrm{~K}^{\circ} \\
y & { }^{6} \mathrm{P}^{\circ} \\
w^{4} \mathrm{~F}^{\circ} \\
w^{4} \mathrm{H}^{\circ}\end{array}$ & $\begin{array}{l}1^{1 / 2} \\
7^{1 / 2} \\
2^{1 / 2} \\
2^{1 / 2} \\
3^{1 / 2}\end{array}$ & $\begin{array}{l}72786.0 ? \\
72903.0 \\
72908.8 \\
72970.1 \\
73142.1\end{array}$ & $\begin{array}{l}\text { 1. } 34 \\
\text { 1. } 07 \\
\text { 1. } 712 \\
0.984 \\
1.32\end{array}$ & $\begin{array}{l}\text { 1. } 067 \\
\text { 1. } 886 \\
\text { 1. } 029 \\
\text { 1. } 238\end{array}$ \\
\hline $\begin{array}{l}4 d^{6}\left({ }^{3} \mathrm{P}\right) 5 p \\
4 d^{6}\left({ }^{3} \mathrm{H}\right) 5 p \\
4 d^{6}\left({ }^{3} \mathrm{H}\right) 5 p \\
4 d^{6}\left({ }^{3} \mathrm{H}\right) 5 p \\
4 d^{6}\left({ }^{3} \mathrm{P}\right) 5 p\end{array}$ & $\begin{array}{ll}z & { }^{2} \mathrm{D}^{\circ} \\
z & 4 \mathrm{I}^{\circ} \\
y & { }^{4} \mathrm{~F}^{\circ} \\
z & { }^{4} \mathrm{I}^{\circ} \\
y & { }^{4} \mathrm{P}^{\circ}\end{array}$ & $\begin{array}{l}21 / 2 \\
71 / 2 \\
4^{1 / 2} \\
5^{1 / 2} \\
11 / 2\end{array}$ & $\begin{array}{l}65244.7 \\
65259.8 \\
65326.7 \\
65472.1 \\
65508.1\end{array}$ & $\begin{array}{l}\text { 1. } 324 \\
\text { 1. } 205 \\
\text { 1. } 152 \\
\text { 1. } 046 \\
\text { 1. } 85 \text { ? }\end{array}$ & $\begin{array}{l}\text { 1. } 200 \\
\text { 1. } 200 \\
\text { 1. } 333 \\
\text { 0. } 965 \\
\text { 1. } 733\end{array}$ & $\begin{array}{l}4 d^{5}\left({ }^{3} \mathrm{D}\right) 5 p \\
4 d^{5} 5 s\left({ }^{7} \mathrm{~S}\right) 5 p \\
4 d^{6}\left({ }^{3} \mathrm{D}\right) 5 p \\
4 d^{6}\left({ }^{3} \mathrm{D}\right) 5 p \\
4 d^{6}\left({ }^{3} \mathrm{D}\right) 5 p\end{array}$ & $\begin{array}{l}x{ }^{4} \mathrm{P}^{\circ} \\
y{ }^{6} \mathrm{P}^{\circ} \\
w^{4} \mathrm{~F}^{\circ} \\
w^{4} \mathrm{D}^{\circ} \\
w^{4} \mathrm{D}^{\circ}\end{array}$ & $\begin{array}{l}0^{1 / 2} \\
31 / 2 \\
41 / 2 \\
21 / 2 \\
11 / 2\end{array}$ & $\begin{array}{l}\text { 73195. } 4 \\
\text { 73213.2 } \\
\text { 73218. } 3 \\
\text { 73284. } \\
\text { 73315. }\end{array}$ & $\begin{array}{l}\text { 1. } 749 \\
\text { 1. } 57 \\
\text { 1. } 28 \\
\text { 1. } 301 \\
\text { 1. } 214\end{array}$ & $\begin{array}{l}\text { 2. } 667 \\
\text { 1. } 714 \\
\text { 1. } 333 \\
\text { 1. } 371 \\
\text { 1. } 200\end{array}$ \\
\hline $\begin{array}{l}4 d^{6}\left({ }^{3} \mathrm{H}\right) 5 p \\
4 d^{6}\left({ }^{3} \mathrm{H}\right) 5 p \\
4 d^{6}\left({ }^{3} \mathrm{~F}\right) 5 p \\
4 d^{6}\left({ }^{3} \mathrm{H}\right) 5 p \\
4 d^{6}\left({ }^{3} \mathrm{H}\right) 5 p\end{array}$ & $\begin{array}{ll}z & 2 \mathrm{I}^{\circ} \\
z & 2 \mathrm{G}^{\circ} \\
z & 2 \mathrm{~F}^{\circ} \\
z & 4 \mathrm{I}^{\circ} \\
z & 2 \mathrm{I}^{\circ}\end{array}$ & $\begin{array}{l}61 / 2 \\
41 / 2 \\
31 / 2 \\
61 / 2 \\
51 / 2\end{array}$ & $\begin{array}{l}65546.5 \\
65776.1 \\
66012.4 \\
66198.6 \\
66249.3\end{array}$ & $\begin{array}{l}\text { 1. } 14 \\
\text { 0. } 991 \\
\text { 1. } 199 \\
\text { 1. } 09 \\
\text { 1. } 04\end{array}$ & $\begin{array}{l}\text { 1. } 077 \\
\text { 1. } 111 \\
\text { 1. } 143 \\
\text { 1. } 108 \\
0.923\end{array}$ & $\begin{array}{l}4 d^{6}\left({ }^{1} \mathrm{I}\right) 5 p \\
4 d^{6}\left({ }^{3} \mathrm{D}\right) 5 p \\
4 d^{6}\left({ }^{3} \mathrm{D}\right) 5 p \\
4 d^{6}\left({ }^{1} \mathrm{I}\right) 5 p \\
4 d^{6}\left({ }^{3} \mathrm{G}\right) 5 p\end{array}$ & $\begin{array}{ll}x & { }^{2} \mathrm{H}^{\circ} \\
w^{4} \mathrm{D}^{\circ} \\
x & { }^{2} \mathrm{G}^{\circ} \\
x & { }^{2} \mathrm{H}^{\circ} \\
x & { }^{2} \mathrm{G}^{\circ}\end{array}$ & $\begin{array}{l}41 / 2 \\
01 / 2 \\
31 / 2 \\
51 / 2 \\
41 / 2\end{array}$ & $\begin{array}{l}73363.4 \\
73750.0 \\
73810.0 \\
73950.6 \\
74045.7\end{array}$ & $\begin{array}{l}0.96 \\
0.552 \\
1.114 \\
1.07 \\
0.809\end{array}$ & $\begin{array}{l}0.909 \\
0.000 \\
0.889 \\
1.091 \\
1.111\end{array}$ \\
\hline $\begin{array}{l}4 d^{6}\left({ }^{3} \mathrm{P}\right) 5 p \\
4 d^{6}\left({ }^{3} \mathrm{P}\right) 5 p \\
4 d^{6}\left({ }^{3} \mathrm{~F}\right) 5 p \\
4 d^{6}\left({ }^{3} \mathrm{~F}\right) 5 p \\
4 d^{6}\left({ }^{3} \mathrm{~F}\right) 5 p\end{array}$ & $\begin{array}{ll}y{ }^{4} \mathrm{D}^{\circ} \\
y & { }^{4} \mathrm{D}^{\circ} \\
x & { }^{4} \mathrm{D}^{\circ} \\
z & { }^{2} \mathrm{~F}^{\circ} \\
x & { }^{4} \mathrm{D}^{\circ}\end{array}$ & $\begin{array}{l}31 / 2 \\
01 / 2 \\
21 / 2 \\
21 / 2 \\
11 / 2\end{array}$ & $\begin{array}{l}66338.0 \\
66633.3 \\
66651.0 \\
67099.3 \\
67103.0\end{array}$ & $\begin{array}{l}\text { 1. } 343 \\
0.789 \\
\text { 1. } 279 \\
0.855 \\
\text { 1. } 187\end{array}$ & $\begin{array}{l}\text { 1. } 429 \\
0.000 \\
\text { 1. } 371 \\
0.857 \\
\text { 1. } 200\end{array}$ & $\begin{array}{l}4 d^{6}\left({ }^{3} \mathrm{D}\right) 5 p \\
4 d^{6}\left({ }^{3} \mathrm{D}\right) 5 p \\
4 d^{6}\left({ }^{3} \mathrm{D}\right) 5 p \\
4 d^{6}\left({ }^{1} \mathrm{G}\right) 5 p \\
4 d^{6}\left({ }^{1} \mathrm{G}\right) 5 p\end{array}$ & $\begin{array}{l}x{ }^{2} \mathrm{D}^{\circ} \\
w^{4} \mathrm{D}^{\circ} \\
x^{2} \mathrm{D}^{\circ} \\
w^{2} \mathrm{G}^{\circ} \\
w^{2} \mathrm{G}^{\circ}\end{array}$ & $\begin{array}{l}11 / 2 \\
31 / 2 \\
21 / 2 \\
41 / 2 \\
31 / 2\end{array}$ & $\begin{array}{l}74592.2 \\
74594.0 \\
7511 \% .4 \\
75346.3 \\
75431.7\end{array}$ & $\begin{array}{l}0.87 \\
\text { 1. } 134 \\
1.20 \\
1.03 \\
0.913\end{array}$ & $\begin{array}{l}0.800 \\
\text { 1. } 429 \\
\text { 1. } 200 \\
\text { 1. } 111 \\
0.889\end{array}$ \\
\hline $\begin{array}{l}4 d^{6}\left({ }^{3} \mathrm{~F}\right) 5 p \\
4 d^{6}\left({ }^{3} \mathrm{H}\right) 5 p \\
\left.4 d^{6}{ }^{3} \mathrm{~F}\right) 5 p \\
4 d^{6}\left({ }^{3} \mathrm{~F}\right) 5 p \\
4 d^{6}\left({ }^{3} \mathrm{P}\right) 5 p\end{array}$ & 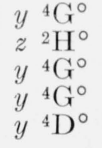 & $\begin{array}{l}4^{1 / 2} \\
5^{1 / 2} \\
5^{1 / 2} \\
3^{1 / 2} \\
11 / 2\end{array}$ & $\begin{array}{l}67165.3 \\
67501.4 \\
67601.7 \\
67646.1 \\
67900.1\end{array}$ & $\begin{array}{l}\text { 1. } 158 \\
\text { 1. } 13 \\
\text { 1. } 166 \\
\text { 1. } 02 \\
\text { 1. } 234\end{array}$ & $\begin{array}{l}\text { 1. } 172 \\
\text { 1. } 091 \\
\text { 1. } 273 \\
\text { 0. } 984 \\
\text { 1. } 200\end{array}$ & $\begin{array}{l}4 d^{6}\left({ }^{1} \mathrm{G}\right) 5 p \\
4 d^{6}\left({ }^{1}\right) 5 p \\
4 d^{6}\left({ }^{1}\right) 5 p \\
4 d^{6}\left({ }^{1} \mathrm{G}\right) 5 p \\
4 d^{6}\left({ }^{1} \mathrm{G}\right) 5 p\end{array}$ & $\begin{array}{l}w^{2} \mathrm{H}^{\circ} \\
y^{2} \mathrm{I}^{\circ} \\
y^{2} \mathrm{I}^{\circ} \\
w^{2} \mathrm{H}^{\circ} \\
w^{2} \mathrm{~F}^{\circ}\end{array}$ & $\begin{array}{l}5^{1 / 2} \\
61 / 2 \\
51 / 2 \\
41 / 2 \\
31 / 2\end{array}$ & $\begin{array}{l}75662.1 \\
75789.5 \\
75925.4 \\
76389.0 \\
7697 \% .5\end{array}$ & $\begin{array}{l}1.08 \\
1.08 \\
0.98 \\
0.93 \\
1.14\end{array}$ & $\begin{array}{l}\text { 1. } 091 \\
1.077 \\
0.923 \\
0.909 \\
1.143\end{array}$ \\
\hline
\end{tabular}


TABLE 2. Odd levels of Ru II-Continued

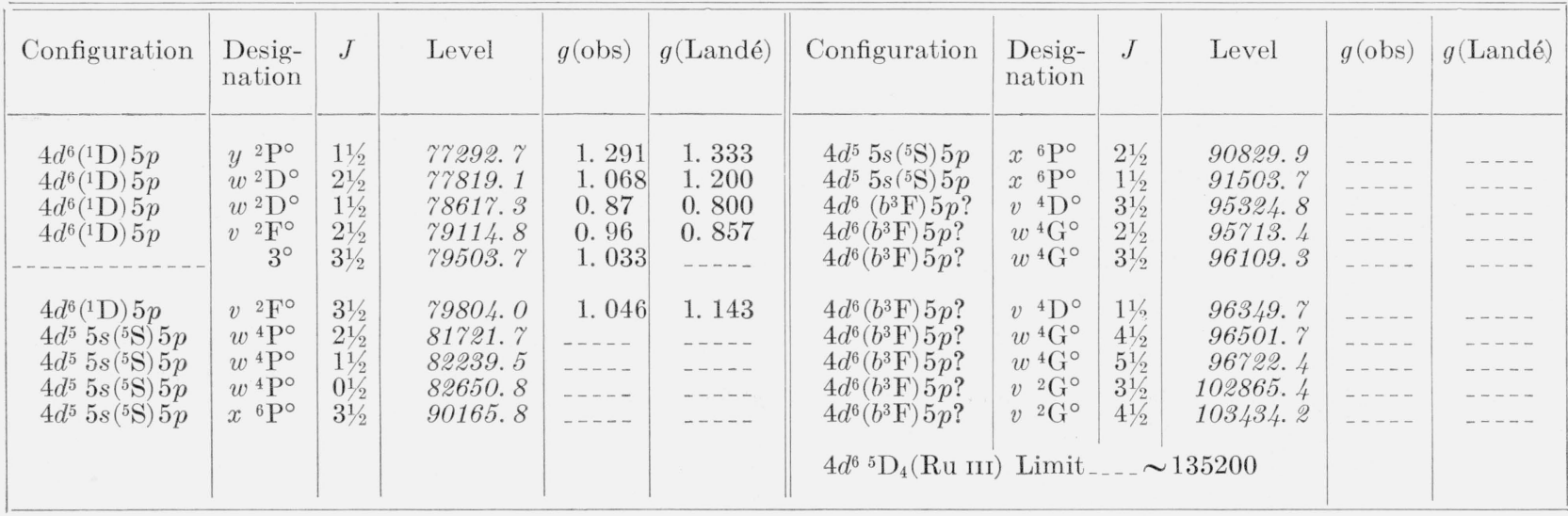

Table 3. Ru II

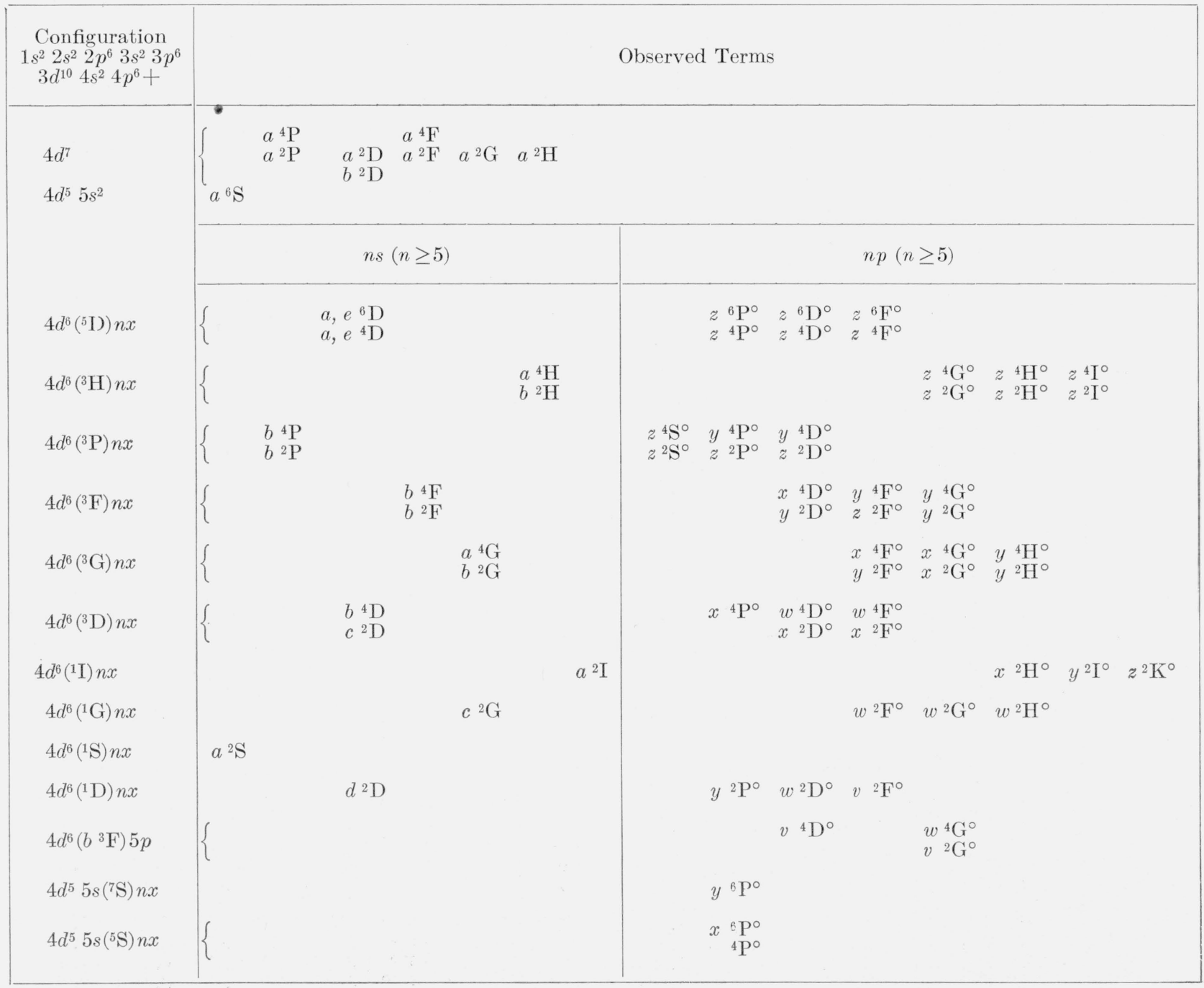


TABLE 4. Numbers of levels of each $J$-value of the structure $4 d^{6} 5 p$ below $80000 \mathrm{~cm}^{-1}$

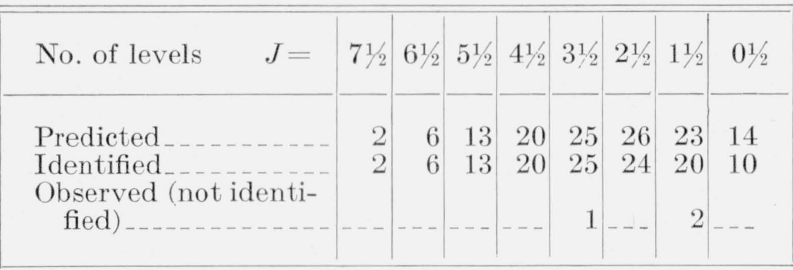

$b^{4} \mathrm{~F}$ have quite regular intervals in $\mathrm{Fe}$ II but in $\mathrm{Ru}$ II they are so irregular that parts of the terms at first eluded discovery. Figure 1 shows these unusual intervals in the terms $a{ }^{4} \mathrm{H}, b{ }^{4} \mathrm{~F}, b^{4} \mathrm{P}$ and $a^{4} \mathrm{G}$. Of considerable assistance, however, was the fact that the positions of the sextet and quartet terms of $\mathrm{d}^{6} s$ in Fe II when multiplied by $3 / 4$ give a rather close correspondence with the same terms in Ru II. The differences between the higher and lower multiplicities based on the same ion term is greater by an average factor of about $1.30 \mathrm{in} \mathrm{Ru} \mathrm{II.}$

The naming of the levels was based on relative intensities of combination, on the Zeeman effect, and on the comparison with Fe II. The various criteria were frequently at variance and compromises were therefore necessary. It will be noticed that hardly any of the $g$-values are in close agreement with the Landé values, and that the values tend to have less variation from the mean than do the Landé values. The worst disagreements are for the levels $x^{4} D_{31 / 2}^{\circ}$ and $y^{4} \mathrm{D}_{01 / 2}^{\circ}$, and it is quite probable that these levels are either misnamed or unaccountably perturbed.

In $\mathrm{Fe}$ II there are known a number of even levels based on the high terms ${ }^{3} \mathrm{~F},{ }^{3} \mathrm{P},{ }^{1} \mathrm{~F},{ }^{1} \mathrm{G}$ from $d^{6}$ in Fe III. A thorough search has been made for the equivalent levels in $\mathrm{Ru}$ II without success, although there remain unidentified a number of lines which could be combinations of such levels with known terms. This failure is all the stranger when it is noticed that some odd terms of this same group have apparently been discovered. It is possible that the observations are not sufficiently exhaustive. Some plates taken at Princeton definitely show a considerably increased number of lines.

Tables 1 and 2, respectively, list all of the known even and odd levels in order of magnitude, and Table 3 relates them to the atomic structure. The levels are all given Russell-Saunders names except three odd levels which are numbered. Table 4 lists the numbers of $4 d^{6} 5 p$ levels to be expected with each $J$-value in the region below $80000 \mathrm{~cm}^{-1}$. It is satisfactory that the numbers discovered are so nearly those predicted, but two discrepancies must be noted. The most striking is the presence of an extra level of $J=3 \frac{1}{2}$. A quite thorough examination of all the $J=3 \frac{1}{2}$ levels has been made and no one of them appears in any way spurious or to have been given an incorrect $J$-value. The two unidentified levels of $J=1 \frac{1}{2}$ do not present such a serious problem since there is room for them numerically even if they do not fit the names and positions of any of the missing levels. The $J=2 \frac{1}{2}$ column lacks two levels, and the $J=0 \frac{1}{2}$ four levels, but the latter fact is not surprising because such levels are always difficult to find.

It was satisfactory to find the $4 d^{5} 5 s^{2}{ }^{6} \mathrm{~S}$ level and three of its associated $\mathrm{P}^{\circ}$ terms, $y, x^{6} \mathrm{P}^{\circ}$ and $w^{4} \mathrm{P}^{\circ}$. The odd terms are confirmed by strong multiplets in combination with $a^{6} \mathrm{D}$, and the ${ }^{6} \mathrm{~S}$ itself combines with $z^{6} \mathrm{P}^{\circ}$. The ${ }^{4} \mathrm{P}^{\circ}$ term of the odd structure was found from its combinations with $a^{4} \mathrm{D}$ in the Schumann region. An unsuccessful search was made for $4 d^{5} 5 s\left({ }^{7} \mathrm{~S}\right) 5 p^{8} \mathrm{P}^{\circ}$.

Most of the data in tables 1 and 2 were supplied in October 1957 for inclusion in Volume III of Atomic Energy Levels [9], where all the levels are grouped in spectral terms and the terms themselves are arranged in order of magnitude of the lowest level of each. This style of presentation has been used consistently in Atomic Energy Levels; it has the advantage of displaying directly the intervals between levels of terms and thus showing the actual splitting as well as whether any complex term is normal, inverted, or partially inverted. Since some slight revisions of level values, intervals, and $g$-factors for $\mathrm{Ru}$ II have been made during the past year our final results are shown in table 5 in the same style as in Atomic Energy Levels [9]. A large majority of the $\mathrm{Ru}$ II terms are inverted as expected, because they arise from configurations containing six or seven type- $d$ electrons, that is, the $d$-shell is more than half filled. However, some terms produced by configurations containing six $d$-electrons are normal or only partially inverted, and even $d^{7}$, expected to give inverted terms, yields two terms, $a^{2} \mathrm{~F}$ and $b^{2} \mathrm{D}$, that are erect instead of inverted.

For pure LS coupling the intervals in complex terms are proportional to the larger $J$-values defining an interval. Thus, for $4 d^{7} a^{4} \mathrm{~F}$ the intervals should be proportional to $4.5: 3.5: 2.5$ whereas they are actually in ratio $4.5: 2.9: 1.8$. Similarly, the intervals between levels of $4 d^{6}\left({ }^{5} \mathrm{D}\right) 5 s \quad a{ }^{6} \mathrm{D}$ should be proportional to $4.50: 3.50: 2.50: 1.50$, but their ratios are observed to be $4.50: 3.16: 2.04: 1.36$. The irregular distribution of levels in four $4 d^{6} 5 s$ terms is illustrated in figure 1 . The remaining $\mathrm{Ru}$ II terms with three or more levels all flagrantly violate the interval rule. These violations, as well as the departures of $g$-factors from the Landé values, indicate that $L S$ coupling is far from perfect in the ruthenium ion but nevertheless strong enough to permit plausible designations of the spectral terms.

The line list (table 6) is given complete although a considerable number of lines remain unidentified. The majority of these are in the Schumann region and many are of large intensity. They probably have their origin in terms of the $4 d^{5} 5 s 5 p$ structure, a few of which have been found. At 1937 A there is a pair of very strong lines which have been worked over very completely, but unsuccessfully. In the longer wavelength region there is a scattering of unidentified lines of some intensity and even two with resolved Zeeman patterns. They may be due to terms built on high levels of $3 d^{6}$ or to terms of $3 d^{5} 4 s^{2}$. A few lines have been remeasured on plates taken in Princeton on high dispersion and have been separated into pairs or threes. 


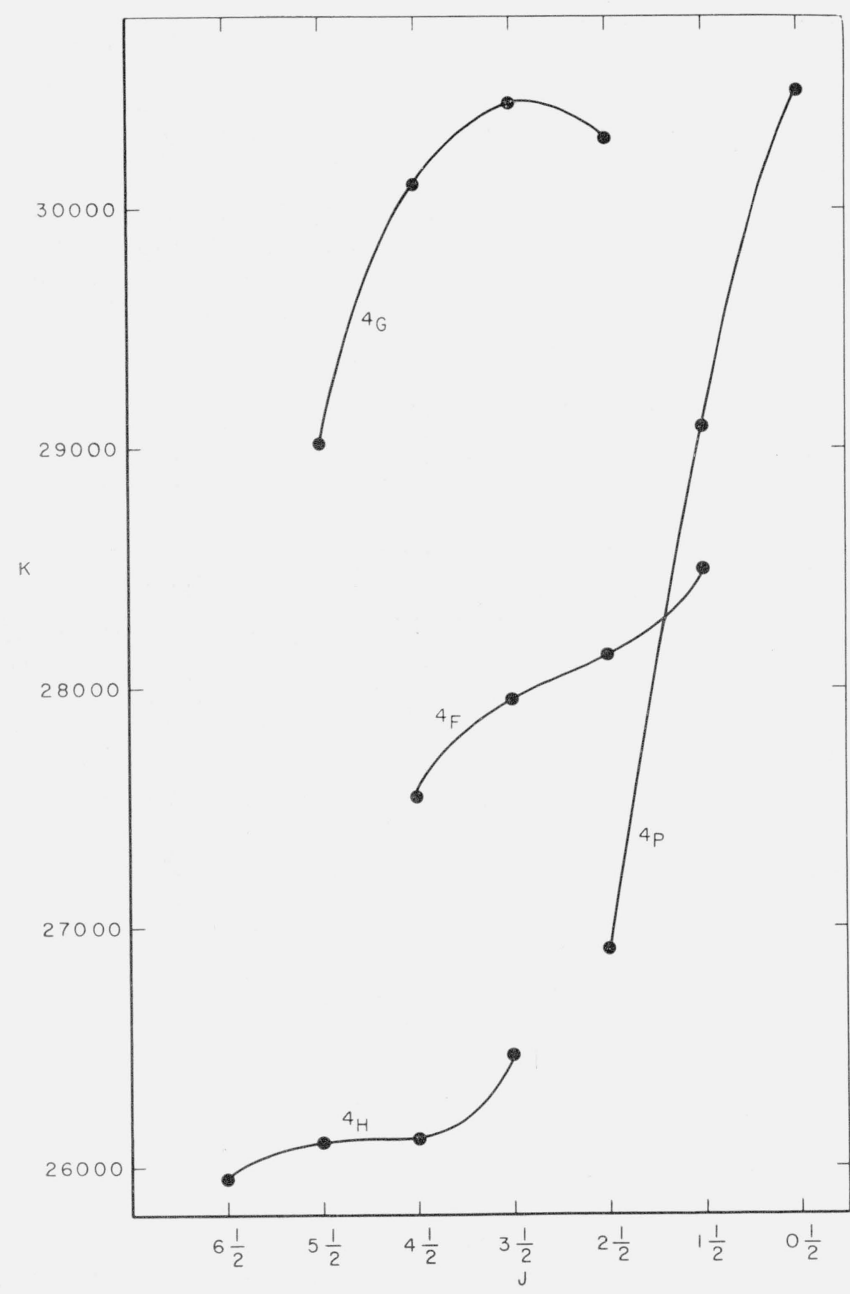

Figure 1. A plot of four Ru II terms from $4 d^{6} 5 \mathrm{~s}$.

Table 6 contains the measured wavelengths and estimated relative intensities of 2,227 spectral lines ascribed to $\mathrm{Ru}$ II in the wavelength range 1054.684 to $6371.29 \mathrm{~A}$. The wavelengths shorter than 2000 A are valid for vacuum, but the longer ones are proper for standard air. For purposes of analysis and interpretation all wavelengths have been converted to vacuum wavenumbers, $\sigma$, in $K\left(=\mathrm{cm}^{-1}\right)$ units in column 3. The 4th column contains the designations of two energy levels (tables $1,2,5$ ) which differ in value by the amount shown in column 3.

For wavelengths greater than 2322 A six additional columns are required in table 6 to report the Zeeman data for $488 \mathrm{Ru}$ II lines. Instead of giving all the observed components of each Zeeman pattern, only the strongest $p$ - and $n$-components are reported in columns 9 and 10 , respectively. This information together with the Zeeman-type numbers [5] in column 5 , and the $g$-factors in columns 7 and 8 corresponding to the $J$-values in column 4 , permits the reconstruction of the complete Zeeman pattern. Actually the absolute values of $J$ and $g$ were first derived from fully observed and resolved Zeeman patterns indicated by $\Delta g$ in column 6 . Later, when many more lines were connected by the usual methods of interval analysis, most of the unresolved Zeeman patterns could be interpreted as confirming the analysis and extending the system of $g$-factors. Only a few Zeeman patterns were too unsymmetrical $(u s)$ to be useful. There are only two resolved and four unresolved Zeeman patterns that appear to disagree with the classification indicated in column 4. These are marked with asterisks accompanying the type number; they may be ascribed to errors of observation in a rich mixture of Zeeman patterns belonging to $\mathrm{RuI}, \mathrm{Ru}$ II, and to impurities.

The total number of $\mathrm{Ru}$ II lines classified in table 6 is 1,633 , and, excepting the spectral range 1054 to $1400 \mathrm{~A}$, there remain relatively few lines of any importance to explain.

Series Limit. Because only two members of the $4 d^{6} n s{ }^{6} \mathrm{D}$ series are known, an accurate limit cannot be calculated directly because such series usually obey the Ritz formula

$$
\frac{T}{4}=\frac{R}{\left(n+\mu+\alpha \frac{T}{4}\right)^{2}},
$$

and the constant $\alpha$ is by no means negligible. An estimate of the value of $\alpha$ is, therefore, necessary and this can be obtained only by a general comparison of spectra of both the first and second long periods. Catalán and Rico [10] have made such a comparison and they deduce a value of $\alpha=4.09 \times 10^{-6}$. With this value and the correct value of the separation between $a^{6} \mathrm{D}$ and $e^{6} \mathrm{D}$, the limit is at $135200 \mathrm{~cm}^{-1}$ to the nearest $100 \mathrm{~cm}^{-1}$, giving an I. P. of $16.76 \mathrm{~V}$.

In a private communication the late Professor Catalán furnished a term table of RhIII, a spectrum isoelectronic with RuII and Tcr. The agreement is excellent in the parts based on the ${ }^{5} \mathrm{D}$ ion, but some of the $4 d^{7}$ terms and many of the high odd and even terms show a considerable deviation from the positions which would be predicted from our analysis of Ruir.

\section{Referencєs}

[1] W. F. Meggers and A. G. Shenstone, Phys. Rev. 35, 868 (1930).

[2] K. G. Kessler and W. F. Meggers, J. Research NBS 55, 97 (1955) RP 2609.

[3] J. C. Dobbie, Ann. Solar Phys. Obs., Cambridge, Engl. 5, Part 1, 1 (1938)

[4] G. R. Harrison and J. R. MeNally, Jr., Phys. Rev. 58, $703(1940))$.

[5] E. Back and A. Landé, Zeemaneffekt und Multiplettstruktur der Spektrallinien, 168, (Julius Springer, Berlin, Germany (1925)).

[6] A. G. Shenstone and H. A. Blair, Phil. Mag. [VIII], 765 (1929).

[7] C. E. Moore, Atomic Energy Levels, NBS Circ. 467, III, $19(1958)$.

[8] C. E. Moore, Atomic Energy Levels, NBS Circ. 467, II, 55 1952).

[9] C. E. Moore, Atomic Energy Levels, NBS Circ. 467, III, 25 (1958).

[10] M. A. Catalán and F. R. Rico, Anales real soc. espan. fis. y quim. (Madrid) [A] 48, 328 (1952). 
TABLE 5. Terms and intervals of RuII

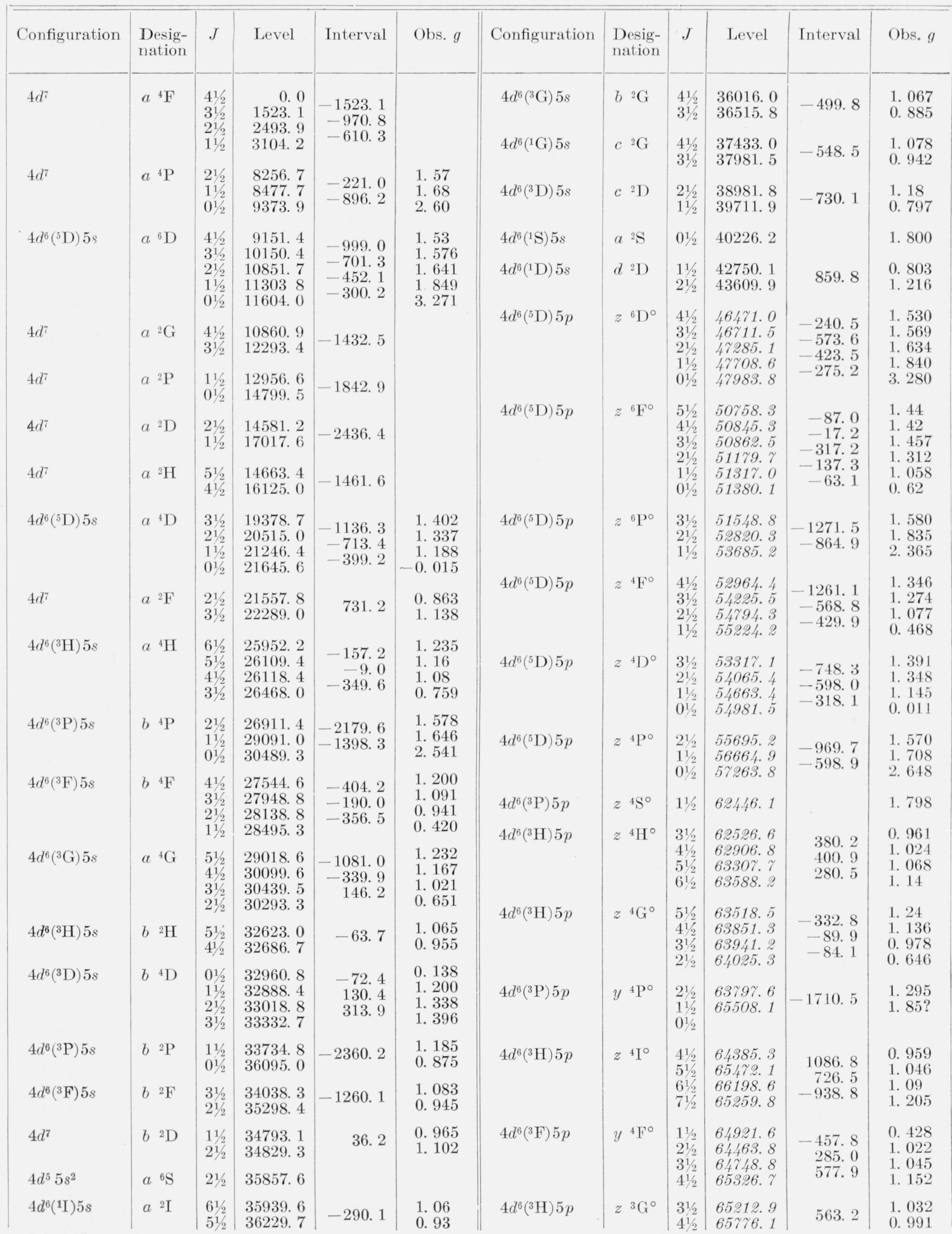


TABLE 5. Terms and intervals of Ru II-Continued

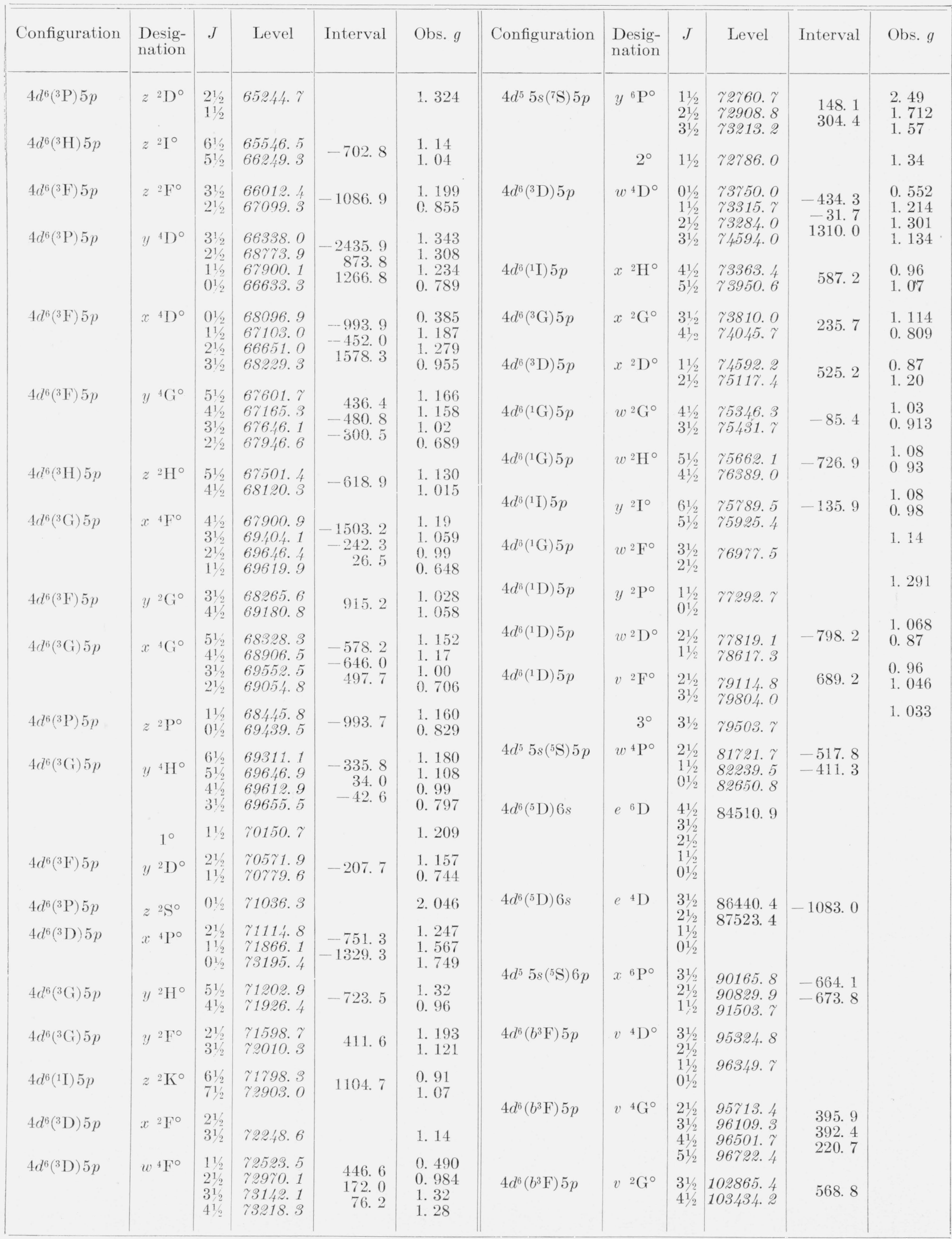


TABLE 6. The second spectrum of ruthenium (Ru II)

\begin{tabular}{|c|c|c|c|c|c|c|c|}
\hline $\begin{array}{c}1 \\
\text { Intensity }\end{array}$ & $\begin{array}{c}2 \\
\lambda(\text { vacuum })\end{array}$ & $\begin{array}{l}3 \\
\sigma\end{array}$ & $\frac{4}{\text { Term combination }}$ & $\begin{array}{c}1 \\
\text { Intensity }\end{array}$ & $\begin{array}{c}2 \\
\lambda(\text { vacuum })\end{array}$ & $\begin{array}{l}3 \\
\sigma\end{array}$ & $\frac{4}{\text { Term combination }}$ \\
\hline $\begin{array}{r}10 \\
0 \\
10 \\
1 \\
1\end{array}$ & $\begin{array}{c}A \\
1054.684 \\
1066.361 \\
1069.266 \\
1074.380 \\
1080.243\end{array}$ & $\begin{array}{c}K \\
94815.1 \\
93776.9 \\
93522.1 \\
93076.9 \\
92571.8\end{array}$ & $a^{2} \mathrm{G}_{41 / 2}-v^{2} \mathrm{G}_{41 / 2}^{\circ}$ & $\begin{array}{r}2 \\
5 \\
50 \\
50 \\
15\end{array}$ & $\begin{array}{c}A \\
1242.580 \\
1243.176 \\
1245.657 \\
1246.887 \\
1249.774\end{array}$ & $\begin{array}{c}K \\
80477.7 \\
80439.1 \\
80278.9 \\
80199.7 \\
80014.5\end{array}$ & $a^{6}{ }^{4} \mathrm{~F}_{31 / 2}-w^{4} \mathrm{P}_{31 / 2}^{0}-x{ }^{6} \mathrm{P}_{31 / 2}^{0}$ \\
\hline $\begin{array}{r}0 \\
2 \\
2 \\
15 \\
1\end{array}$ & $\begin{array}{l}1080.945 \\
1081.684 \\
1083.701 \\
1093.505 \\
1101.132\end{array}$ & $\begin{array}{l}92511.6 \\
92448.4 \\
92276.4 \\
91449.1 \\
90815.6\end{array}$ & - & $\begin{array}{r}3 \\
20 \\
5 \\
15 \\
50\end{array}$ & $\begin{array}{l}1250.171 \\
1250.340 \\
1251.174 \\
1251.582 \\
1252.789\end{array}$ & $\begin{array}{l}79989.1 \\
79978.2 \\
79924.9 \\
79898.9 \\
79821.9\end{array}$ & $a^{6} \mathrm{D}_{21 / 2}-x^{6} \mathrm{P}_{21 / 2}$ \\
\hline $\begin{array}{r}10 \\
15 \\
10 \\
5 \\
2\end{array}$ & $\begin{array}{l}1103.223 \\
1105.125 \\
1109.227 \\
1117.906 \\
1139.255\end{array}$ & $\begin{array}{l}90643.5 \\
90487.5 \\
90152.9 \\
89453.0 \\
87776.7\end{array}$ & 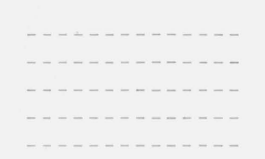 & $\begin{array}{l}50 \\
50 \\
20 \\
30 \\
10\end{array}$ & $\begin{array}{l}1253.419 \\
1253.622 \\
1254.717 \\
1255.329 \\
1255.430\end{array}$ & $\begin{array}{l}79781.8 \\
79768.9 \\
79699.2 \\
79660.4 \\
79654.0\end{array}$ & - \\
\hline $\begin{array}{l}1 \\
1 \\
1 \\
5 \\
2\end{array}$ & $\begin{array}{l}1144.504 \\
1147.835 \\
1153.309 \\
1162.357 \\
1163.330\end{array}$ & $\begin{array}{l}87374.1 \\
87120.5 \\
86707.0 \\
86032.1 \\
85900.1\end{array}$ & 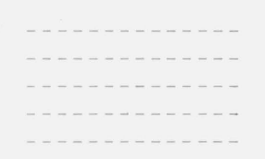 & $\begin{array}{r}2 \\
2 \\
40 \\
15 \\
20\end{array}$ & $\begin{array}{l}1256.082 \\
1256.898 \\
1257.443 \\
1258.127 \\
1258.351\end{array}$ & $\begin{array}{l}79612.6 \\
79561.0 \\
79526.5 \\
79483.2 \\
79469.1\end{array}$ & $a^{6} \mathrm{D}_{11 / 2} x^{6} \mathrm{P}_{21 / 2}^{0}$ \\
\hline $\begin{array}{l}2 \\
1 \\
1 \\
1 \\
1\end{array}$ & $\begin{array}{l}1163.537 \\
1169.846 \\
1176.783 \\
1182.233 \\
1186.583\end{array}$ & $\begin{array}{l}85944.8 \\
85481.3 \\
84977.4 \\
84585.7 \\
84275.6\end{array}$ & - & $\begin{array}{l}10 \\
15 \\
50 \\
20 \\
20\end{array}$ & $\begin{array}{l}1259.038 \\
1259.539 \\
1260.502 \\
1260.817 \\
1261.418\end{array}$ & $\begin{array}{l}79425.7 \\
79394.1 \\
79333.5 \\
79313.7 \\
79275.9\end{array}$ & $a^{6} \mathrm{D}_{21 / 2} \quad x^{6} \mathrm{P}_{31 / 2}^{0}$ \\
\hline $\begin{array}{r}100 \\
10 \\
10 \\
10 \\
2\end{array}$ & $\begin{array}{l}1190.243 \\
1190.302 \\
1195.261 \\
1196.983 \\
1197.511\end{array}$ & $\begin{array}{l}84016.5 \\
84012.3 \\
83663.7 \\
83543.4 \\
83506.5\end{array}$ & - & $\begin{array}{r}10 \\
5 \\
10 \\
30 \\
3\end{array}$ & $\begin{array}{l}1262.688 \\
1263.172 \\
1263.396 \\
1263.537 \\
1263.848\end{array}$ & $\begin{array}{l}79196.1 \\
79165.8 \\
79151.7 \\
79142.9 \\
79123.4\end{array}$ & 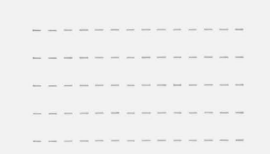 \\
\hline $\begin{array}{r}5 \\
5 \\
50 \\
30 \\
30\end{array}$ & $\begin{array}{l}1199.341 \\
1202.726 \\
1204.564 \\
1204.636 \\
1204.995\end{array}$ & $\begin{array}{l}83379.1 \\
83144.5 \\
83017.6 \\
83012.6 \\
82987.9\end{array}$ & - & $\begin{array}{l}100 \\
15 \\
10 \\
15 ? \\
20\end{array}$ & $\begin{array}{l}1264.726 \\
1265.944 \\
1266.010 \\
1266.099 \\
1267.348\end{array}$ & $\begin{array}{l}79068.5 \\
78992.4 \\
78987.8 \\
78982.8 \\
78904.9\end{array}$ & 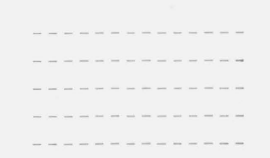 \\
\hline $\begin{array}{r}15 \\
10 \\
50 \\
5 \\
10\end{array}$ & $\begin{array}{l}1205.200 \\
1206.195 \\
1207.811 \\
1208.863 \\
1213.028\end{array}$ & $\begin{array}{l}82973.8 \\
82905.3 \\
82794.4 \\
82722.4 \\
82438.3\end{array}$ & 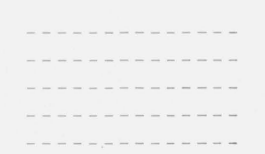 & $\begin{array}{r}10 \\
2 \\
3 \\
2 \\
50\end{array}$ & $\begin{array}{l}1267.674 \\
1268.046 \\
1268.497 \\
1268.776 \\
1269.324\end{array}$ & $\begin{array}{l}78884.6 \\
78861.5 \\
78833.5 \\
78816.1 \\
78782.1\end{array}$ & 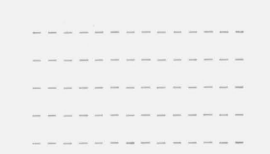 \\
\hline $\begin{array}{r}5 \\
10 \\
5 \\
3 \\
10\end{array}$ & $\begin{array}{l}1213.644 \\
1213.863 \\
1222.201 \\
1223.684 \\
1226.736\end{array}$ & $\begin{array}{l}82396.5 \\
82381.6 \\
81819.6 \\
81720.4 \\
81517.1\end{array}$ & מ & $\begin{array}{r}15 \\
5 \\
3 \\
30 \\
15\end{array}$ & $\begin{array}{l}1270.911 \\
1271.528 \\
1271.653 \\
1271.775 \\
1271.921\end{array}$ & $\begin{array}{l}78683.7 \\
78645.5 \\
78637.8 \\
78630.3 \\
78621.2\end{array}$ & - \\
\hline $\begin{array}{r}5 \\
50 \\
20 \\
2 \\
10\end{array}$ & $\begin{array}{l}1226.867 \\
1227.101 \\
1228.255 \\
1232.670 \\
1232.809\end{array}$ & $\begin{array}{l}81508.4 \\
81492.9 \\
81416.3 \\
81124.7 \\
81115.6\end{array}$ & - & $\begin{array}{r}5 \\
200 \\
300 \\
30 \\
3\end{array}$ & $\begin{array}{l}1273.192 \\
1273.344 \\
1278.543 \\
1280.736 \\
1281.079\end{array}$ & $\begin{array}{l}78542.7 \\
78533.4 \\
78214.0 \\
78080.1 \\
78059.2\end{array}$ & 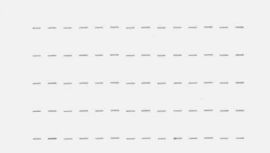 \\
\hline $\begin{array}{r}100 \\
3 \\
10 \\
10 \\
100\end{array}$ & $\begin{array}{l}1234.347 \\
1234.834 \\
1235.247 \\
1239.289 \\
1239.467\end{array}$ & $\begin{array}{l}81014.5 \\
80982.5 \\
80955.5 \\
80691.4 \\
80679.8\end{array}$ & $\frac{a^{6} \mathrm{D}_{41 / 2}-x{ }^{6} \mathrm{P}_{31 / 2}^{0}}{a^{6} \mathrm{D}_{31 / 2} x{ }^{6} \mathrm{P}_{21 / 2}^{0}}$ & $\begin{array}{r}5 \\
20 \\
5 \\
5 \\
5\end{array}$ & $\begin{array}{l}1281.183 \\
1281.419 \\
1282.159 \\
1283.769 \\
1283.981\end{array}$ & $\begin{array}{l}78052.9 \\
78038.5 \\
77993.4 \\
77895.6 \\
77882.8\end{array}$ & 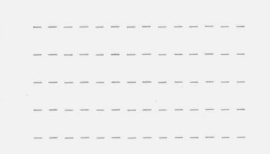 \\
\hline $\begin{array}{r}50 \\
20 \\
3 \\
5 \\
3\end{array}$ & $\begin{array}{l}1239.879 \\
1240.647 \\
1241.920 \\
1242.056 \\
1242.356\end{array}$ & $\begin{array}{l}80653.0 \\
80603.1 \\
80520.5 \\
80511.7 \\
80492.2\end{array}$ & $a{ }^{6} \mathrm{D}_{21 / 2}-x{ }^{6} \mathrm{P}_{11 / 2}^{\circ}$ & $\begin{array}{r}1 \\
5 \\
50 \\
2 \\
5\end{array}$ & $\begin{array}{l}1284.292 \\
1285.006 \\
1285.528 \\
1285.811 \\
1285.923\end{array}$ & $\begin{array}{l}77863.9 \\
77820.6 \\
77789.0 \\
77771.9 \\
77765.2\end{array}$ & - \\
\hline
\end{tabular}


TABLE 6. The second spectrum of ruthenium ( $\mathrm{Ru}$ II) - Continued

\begin{tabular}{|c|c|c|c|c|c|c|c|}
\hline $\begin{array}{c}1 \\
\text { Intensity }\end{array}$ & $\begin{array}{c}2 \\
\lambda(\text { vacuum })\end{array}$ & $\begin{array}{l}3 \\
\sigma\end{array}$ & $\frac{4}{\text { Term combination }}$ & $\begin{array}{c}1 \\
\text { Intensity }\end{array}$ & $\begin{array}{c}2 \\
\lambda(\text { vacuum })\end{array}$ & $\begin{array}{l}3 \\
\sigma\end{array}$ & $\begin{array}{c}4 \\
\text { Term combination }\end{array}$ \\
\hline $\begin{array}{r}10 \\
20 \\
15 \\
5 \\
15\end{array}$ & $\begin{array}{c}A \\
1286.574 \\
1287.965 \\
1288.658 \\
1288.884 \\
1290.177\end{array}$ & $\begin{array}{c}K \\
77725.8 \\
77641.9 \\
77600.1 \\
77586.5 \\
77508.7\end{array}$ & - & $\begin{array}{r}5 \\
2 \\
2 \\
3 \\
20\end{array}$ & $\begin{array}{c}A \\
1360.634 \\
1360.888 \\
1362.346 \\
1364.284 \\
1365.465\end{array}$ & $\begin{array}{c}K \\
73495.2 \\
73481.6 \\
73402.8 \\
73298.5 \\
73235.1\end{array}$ & 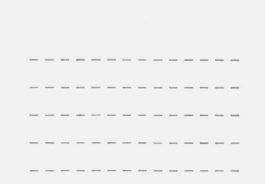 \\
\hline $\begin{array}{r}50 \\
5 \\
15 \\
10 \\
10\end{array}$ & $\begin{array}{l}1290.780 \\
1291.757 \\
1293.009 \\
1295.920 \\
1296.239\end{array}$ & $\begin{array}{l}77472.5 \\
77413.9 \\
77339.0 \\
77165.3 \\
77146.3\end{array}$ & 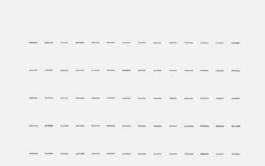 & $\begin{array}{r}5 \\
5 \\
50 \\
30 \\
1\end{array}$ & $\begin{array}{l}1366.434 \\
1368.503 \\
1369.106 \\
1369.852 \\
1376.344\end{array}$ & $\begin{array}{l}73183.2 \\
73072.5 \\
73040.4 \\
73001.3 \\
72657.8\end{array}$ & $a^{4} \mathrm{~F}_{31 / 2}-w^{4} \mathrm{D}_{31 / 2}^{\circ}$ \\
\hline $\begin{array}{r}50 \\
5 \\
5 \\
5 \\
10\end{array}$ & $\begin{array}{l}1296.425 \\
1306.874 \\
1307.325 \\
1307.494 \\
1307.662\end{array}$ & $\begin{array}{l}77135.2 \\
76518.5 \\
76492.1 \\
76482.2 \\
76472.4\end{array}$ & 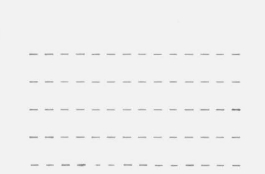 & $\begin{array}{r}2 \\
2 \\
3 \\
10 \\
3\end{array}$ & $\begin{array}{l}1381.703 \\
1382.354 \\
1384.134 \\
1385.133 \\
1386.015\end{array}$ & $\begin{array}{l}72374.8 \\
72340.4 \\
72242.3 \\
72195.2 \\
72149.3\end{array}$ & 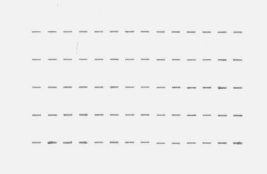 \\
\hline 10 & 1308.162 & 76443.1 & $-6-1,-\infty$ & 5 & 1388.640 & 72012.9 & $\begin{array}{l}a^{4} \mathrm{~F}_{41 / 2}-y{ }^{2} \mathrm{~F}_{31 / 2}^{\circ} \\
a^{4} \mathrm{~F}_{11 / 2}-x \\
{ }^{2} \mathrm{D}_{21 / 2}^{\circ}\end{array}$ \\
\hline $\begin{array}{l}5 \\
2\end{array}$ & 1308.656 & 76414.3 & - & $\begin{array}{r}5 \\
20\end{array}$ & 1392.145 & 71831.6 & $--4-1 / 20-12$ \\
\hline $\begin{array}{r}2 \\
20\end{array}$ & $\begin{array}{l}1311.302 \\
1312.099\end{array}$ & $\begin{array}{l}76260.1 \\
76213.8\end{array}$ & $-\cdots$ & 20 & $\begin{array}{l}1393.535 \\
1396.162\end{array}$ & $\begin{array}{l}71759.9 \\
71624.9\end{array}$ & $a{ }^{4} \mathrm{~F}_{31 / 2}-w^{4} \mathrm{D}_{21 / 2}^{\circ}$ \\
\hline 10 & 1312.557 & 76187.2 & 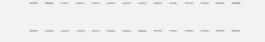 & 2 & 1398.472 & 71506.6 & $\ldots \ldots \ldots$ \\
\hline $\begin{array}{r}50 \\
0 \\
5 \\
2 \\
1\end{array}$ & $\begin{array}{l}1313.384 \\
1314.306 \\
1314.886 \\
1315.624 \\
1315.949\end{array}$ & $\begin{array}{l}76139.2 \\
76085.8 \\
76052.2 \\
76009.6 \\
75990.8\end{array}$ & $a^{4} \mathrm{~F}_{11 / 2}-v^{2} \mathrm{~F}_{21 / 2}^{1}$ & $\begin{array}{r}5 \\
50 \\
10 \\
50 \\
100\end{array}$ & $\begin{array}{l}1399.427 \\
1405.338 \\
1411.991 \\
1412.206 \\
1413.017\end{array}$ & $\begin{array}{l}71457.8 \\
71157.3 \\
70822.0 \\
70811.2 \\
70770.6\end{array}$ & $a^{4} \mathrm{~F}_{21 / 2}-w^{4} \mathrm{D}_{11 / 2}^{\circ}$ \\
\hline 50 & 1316.573 & 75954.8 & $b^{4} \mathrm{P}_{21 / 2}-v^{2} \mathrm{G}_{31 / 2}^{\circ}$ & 5 & 1415.500 & 70646.4 & $\left\{\begin{array}{l}a^{4} \mathrm{~F}_{21 / 2}-w^{4} \mathrm{~F}_{31 / 2}^{0} \\
a^{4} \mathrm{~F}_{11 / 2}-w^{4} \mathrm{D}_{01 / 2}^{\circ}\end{array}\right.$ \\
\hline 20 & 1316.740 & 75945.1 & $a^{4} \mathrm{D}_{31 / 2}^{2 / 2}-v{ }^{4} \mathrm{D}_{31 / 2}^{1}$ & 5 & 1415.678 & 70637.5 & $a^{4} \mathrm{P}_{11 / 2}^{1 / 2}-v{ }^{2} \mathrm{~F}_{21 / 2}^{01 / 2}$ \\
\hline $\begin{array}{l}1 \\
2\end{array}$ & $\begin{array}{l}1320.043 \\
1321.495\end{array}$ & $\begin{array}{l}75755.1 \\
75671.9\end{array}$ & 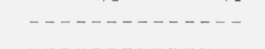 & 10 & 1416.358 & 70603.6 & $a^{4} \mathrm{H}_{41 / 2}-w^{4} \mathrm{G}_{51 / 2}$ \\
\hline 10 & 1324.275 & 75513.0 & $a^{4} \mathrm{~F}_{11 / 2}-w^{2} \mathrm{D}_{11 / 2}^{\circ}$ & $\begin{array}{l}1 \\
2\end{array}$ & $\begin{array}{l}1418.299 \\
1418.909\end{array}$ & 70476.7 & $a^{4} \mathrm{~F}_{21 / 2}-w^{4} \mathrm{~F}_{21 / 2}^{\circ}$ \\
\hline 1 & 1324.654 & 75491.4 & ----------- & 100 & 1420.606 & 70392.5 & $a^{4} \mathrm{H}_{51 / 2}-w^{4} \mathrm{G}_{41 / 2}^{0}$ \\
\hline 3 & 1326.118 & 75408.1 & $-1-10-1$ & 1 & 1420.793 & 70383.2 & $a^{4} \mathrm{H}_{41 / 2}-w^{4} \mathrm{G}_{41 / 2}$ \\
\hline 10 & 1326.318 & 75396.7 & - - - - & $\begin{array}{l}5 \\
3\end{array}$ & 1421.828 & 70332.0 & $\ldots$ \\
\hline $\begin{array}{l}5 \\
1\end{array}$ & $\begin{array}{l}1327.776 \\
1329.403\end{array}$ & $\begin{array}{l}75313.9 \\
75221.7\end{array}$ & $-1+2-10$ & $\begin{array}{l}3 \\
2\end{array}$ & $\begin{array}{l}1422.661 \\
1423.056\end{array}$ & $\begin{array}{l}70290.8 \\
70271.3\end{array}$ & $a^{4} \mathrm{~F}_{21 / 2}^{2}-2_{11 / 2}^{\circ}$ \\
\hline $\begin{array}{l}2 \\
1 \\
1 \\
1 \\
3\end{array}$ & $\begin{array}{l}1329.583 \\
1329.836 \\
1333.018 \\
1334.830 \\
1335.008\end{array}$ & $\begin{array}{l}75211.6 \\
75197.2 \\
75017.7 \\
74915.9 \\
74905.9\end{array}$ & $\frac{a^{4} \mathrm{D}_{21 / 2}-w^{4} \mathrm{G}_{21 / 2}^{0}}{b^{4} \mathrm{~F}_{31 / 2}-v^{2} \mathrm{G}_{31 / 2}^{0}}$ & $\begin{array}{r}5 \\
50 \\
10 \\
2 \\
5\end{array}$ & $\begin{array}{l}1424.272 \\
1424.934 \\
1424.039 \\
1426.391 \\
1426.503\end{array}$ & $\begin{array}{l}70211.3 \\
70178.7 \\
70173.5 \\
70107.0 \\
70101.5\end{array}$ & $\frac{a^{4} \mathrm{~F}_{11 / 2}-w^{4} \mathrm{D}_{11 / 2}^{0}}{b^{2} \mathrm{H}_{41 / 2}-v^{2} \mathrm{G}_{31 / 2}^{0}}$ \\
\hline $\begin{array}{l}5 h \\
20 \\
10 ?\end{array}$ & $\begin{array}{l}1336.023 \\
1338.388 \\
1339.517\end{array}$ & $\begin{array}{l}74849.0 \\
74716.7 \\
74653.8\end{array}$ & - & $\begin{array}{l}3 \\
3 \\
5\end{array}$ & $\begin{array}{l}1426.706 \\
1427.890 \\
1427.964\end{array}$ & $\begin{array}{l}70091.5 \\
70033.4 \\
70029.8\end{array}$ & $\begin{array}{l}a{ }^{4} \mathrm{~F}_{11 / 2}-x{ }^{4} \mathrm{P}_{01 / 2}^{\circ} \\
a^{4} \mathrm{H}_{31 / 2}-w^{4} \mathrm{G}_{41 / 2}^{\circ} \\
a^{4} \mathrm{~F}_{21 / 2}-w^{4} \mathrm{~F}_{11 / 2}^{\circ}\end{array}$ \\
\hline 8 & 1340.165 & 74617.7 & $-1-1-1-1-1$ & 100 & 1428.751 & 69991.2 & $a^{4} \mathrm{H}_{41 / 2}-w^{4} \mathrm{G}_{31 / 2}^{0}$ \\
\hline $\begin{array}{l}15 \\
50\end{array}$ & $\begin{array}{l}1340.590 \\
1340.754\end{array}$ & $\begin{array}{l}74594.0 \\
74584.9\end{array}$ & $a^{4} \mathrm{~F}_{41 / 2}-w^{4} \mathrm{D}_{31 / 2}^{\circ}$ & 30 & 1429.002 & 69978.9 & $\ldots \ldots$ \\
\hline $\begin{array}{l}50 \\
10\end{array}$ & $\begin{array}{l}1340.754 \\
1341.649\end{array}$ & $\begin{array}{l}74584.9 \\
74535.1\end{array}$ & - & $\begin{array}{l}0 \\
2 \\
1\end{array}$ & $\begin{array}{l}1429.348 \\
1431.278 \\
1431.606\end{array}$ & $\begin{array}{l}69962.0 \\
69867.6 \\
69851.6\end{array}$ & $a^{4} \mathrm{~F}_{11 / 2}-w^{4} \mathrm{~F}_{21 / 2}$ \\
\hline 50 & 1342.358 & 74495.8 & $\ldots \ldots \ldots \ldots$ & 1 & 1431.705 & 69846.8 & $b^{4} \mathrm{D}_{21 / 2}-v^{2} \mathrm{G}_{31 / 2}^{\circ}$ \\
\hline $\begin{array}{r}10 \\
3\end{array}$ & $\begin{array}{l}1342.798 \\
1346.839\end{array}$ & $\begin{array}{l}74471.4 \\
74247.9\end{array}$ & - n- & 1 & 1431.819 & 69841.2 & 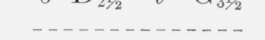 \\
\hline 200 & $\begin{array}{l}1040.859 \\
1348.385\end{array}$ & $\begin{array}{l}74247.9 \\
74162.8\end{array}$ & 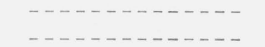 & 2 & 1433.294 & 69769.4 & $\ldots \ldots \ldots \ldots$ \\
\hline 15 & 1350.856 & 74027.1 & 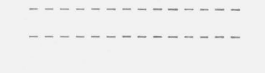 & $\begin{array}{l}30 \\
20\end{array}$ & $\begin{array}{l}1434.330 \\
1435.085\end{array}$ & $\begin{array}{l}69719.0 \\
69682.3\end{array}$ & $a^{4} \mathrm{~F}_{11 / 2}-2_{1 / 2}^{\circ}$ \\
\hline 3 & 1351.857 & 73972.3 & & 20 & 1435.286 & 69672.5 & $-1-1-1-1-1$ \\
\hline 15 & 1353.907 & 73860.3 & - - & 50 & 1437.460 & 69567.2 & - - - - \\
\hline 10 & 1354.835 & 73807.7 & - n & 50 & 1437.839 & 69548.8 & - \\
\hline $\begin{array}{l}2 \\
2\end{array}$ & $\begin{array}{l}1357.221 \\
1357.524\end{array}$ & $\begin{array}{l}73680.0 \\
73663.5\end{array}$ & - n & $\begin{array}{r}1 \\
30\end{array}$ & $\begin{array}{l}1439.001 \\
1440.514\end{array}$ & $\begin{array}{l}69492.7 \\
69419.7\end{array}$ & $a^{4} \mathrm{~F}_{11 / 2}-w^{4} \mathbf{F}_{11 / 6}^{0}$ \\
\hline
\end{tabular}


TABLE 6. The second spectrum of ruthenium (Ru II)-Continued

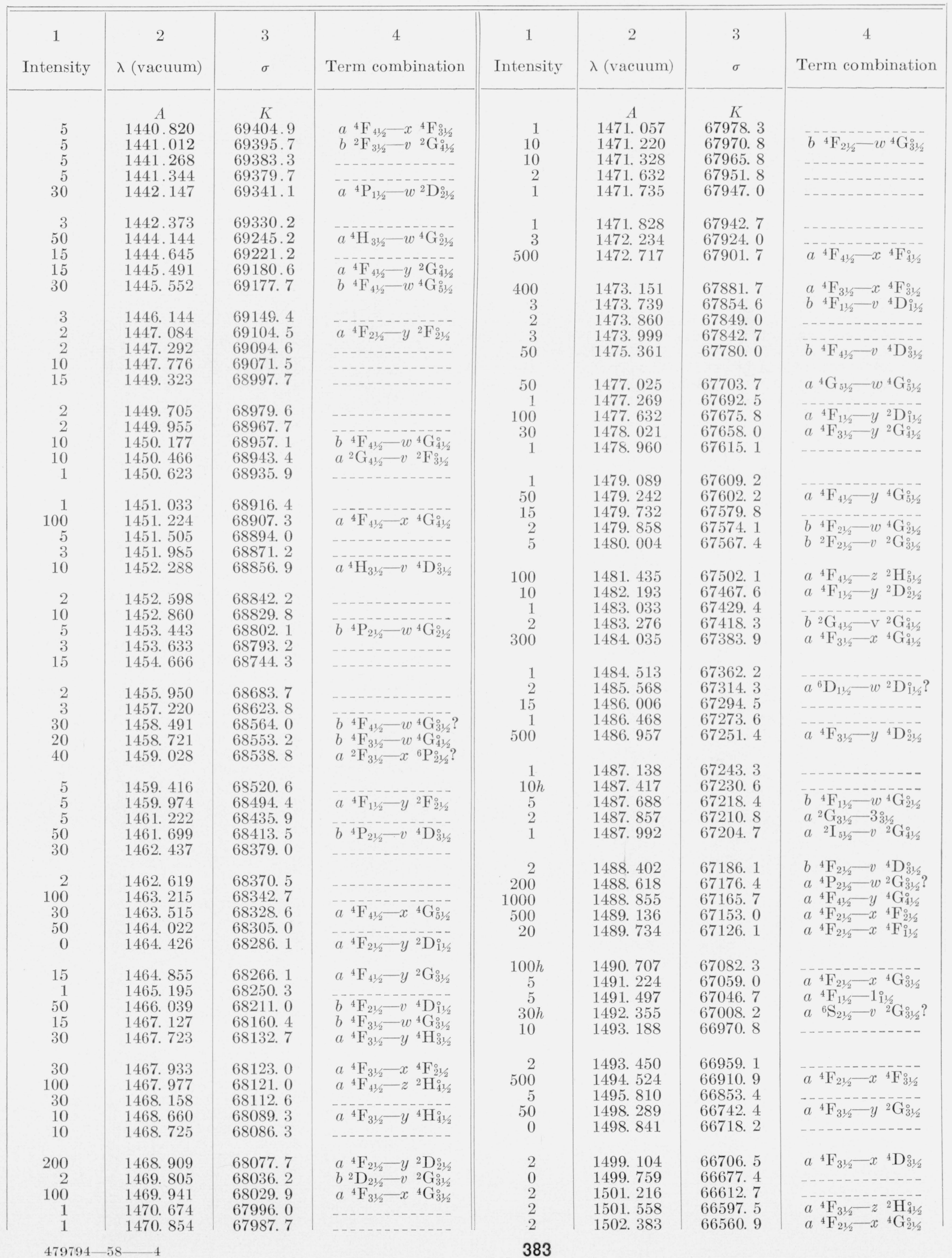


TABLE 6. The second spectrum of ruthenium (Ru II)-Continued

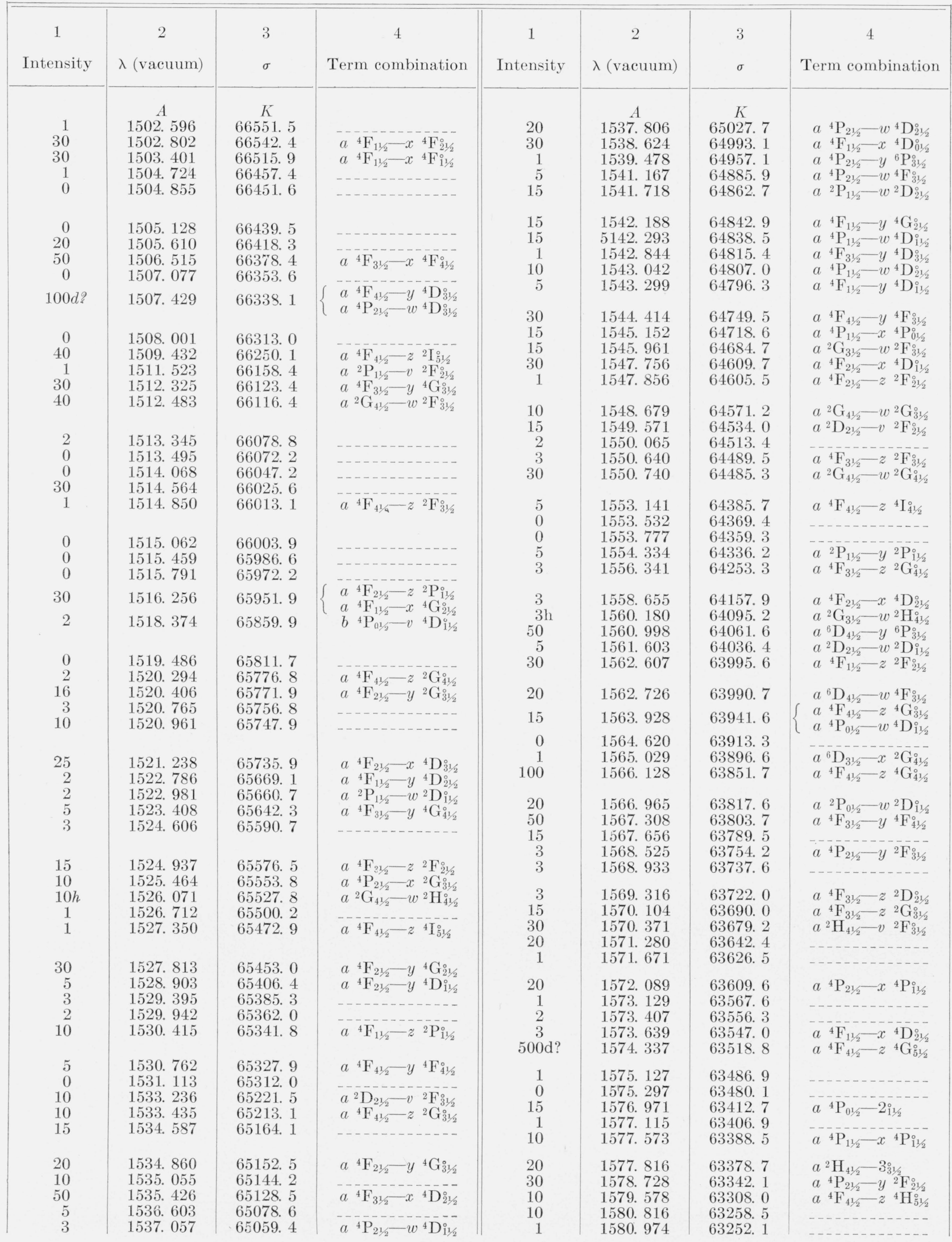


TABLE 6. The second spectrum of ruthenium (Ru II)-Continued

\begin{tabular}{|c|c|c|c|c|c|c|c|}
\hline $\begin{array}{c}1 \\
\text { Intensity }\end{array}$ & $\begin{array}{c}2 \\
\lambda(\text { vacuum })\end{array}$ & $\begin{array}{l}3 \\
\sigma\end{array}$ & $\frac{4}{\text { Term combination }}$ & $\begin{array}{c}1 \\
\text { Intensity }\end{array}$ & $\begin{array}{c}2 \\
\lambda(\text { vacuum })\end{array}$ & $\begin{array}{l}3 \\
\sigma\end{array}$ & $\frac{4}{\text { Term combination }}$ \\
\hline $\begin{array}{r}15 \\
10 \\
5 \\
10 \\
1\end{array}$ & $\begin{array}{c}A \\
1581.328 \\
1581.636 \\
1581.791 \\
1582.652 \\
1582.903\end{array}$ & $\begin{array}{c}K \\
\text { 63238. } 0 \\
63225.7 \\
63219.5 \\
63185.1 \\
63175.1\end{array}$ & $\begin{array}{c}a^{2} \mathrm{D}_{21 / 2}-w^{2} \mathrm{D}_{21 / 2}^{\circ} \\
a^{4} \mathrm{~F}_{31 / 2}-y{ }^{4} \mathrm{~F}_{31 / 2}^{2} \\
a^{2} \mathrm{G}_{41 / 2}-x^{2} \mathrm{G}_{41 / 2}^{\circ}\end{array}$ & $\begin{array}{r}5 \\
20 \\
20 \\
20 \\
1\end{array}$ & $\begin{array}{c}A \\
1621.728 \\
1622.431 \\
1623.231 \\
1623.390 \\
1624.563\end{array}$ & $\begin{array}{c}K \\
61662.6 \\
61635.9 \\
61605.5 \\
61599.5 \\
61555.0\end{array}$ & 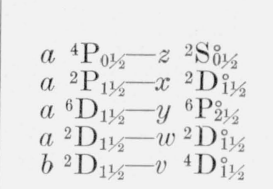 \\
\hline $\begin{array}{r}200 \\
30 \\
50 \\
30 \\
15\end{array}$ & $\begin{array}{l}1583.819 \\
1584.256 \\
1585.040 \\
1585.740 \\
1585.965\end{array}$ & $\begin{array}{l}63138.5 \\
63121.1 \\
63089.9 \\
63062.0 \\
63053.1\end{array}$ & $\begin{array}{l}a^{2} \mathrm{G}_{31 / 2}-w^{2} \mathrm{G}_{31 / 2}^{0} \\
a^{4} \mathrm{P}_{11 / 2}-y \\
a^{2} \mathrm{~F}_{21 / 2}^{0} \\
a^{2} \mathrm{G}_{41 / 2}-x{ }^{2} \mathrm{H}_{51 / 2}^{0} \\
a^{6} \mathrm{D}_{31 / 2}-y{ }^{6} \mathrm{P}_{31 / 2}^{0} \\
a^{2} \mathrm{G}_{31 / 2}-w^{2} \mathrm{G}_{41 / 2}^{0}\end{array}$ & $\begin{array}{r}100 \\
50 \\
50 \\
100 \\
10\end{array}$ & $\begin{array}{l}1625.178 \\
1625.565 \\
1627.138 \\
1627.397 \\
1628.541\end{array}$ & $\begin{array}{l}61531.7 \\
61517.1 \\
61457.6 \\
61447.8 \\
61404.7\end{array}$ & $\begin{array}{l}a{ }^{4} \mathrm{~F}_{21 / 2}-z{ }^{4} \mathrm{G}_{21 / 2}{ }^{\circ 1 / 2} \\
a^{2} \mathrm{G}_{31 / 2}-x{ }^{2} \mathrm{G}_{31 / 2} \\
a^{6} \mathrm{D}_{11 / 2}-y{ }^{6} \mathrm{P}_{11 / 2}^{0} \\
a^{4} \mathrm{~F}_{21 / 2}-z{ }^{4} \mathrm{G}_{31 / 2}^{0} \\
a^{4} \mathrm{D}_{11 / 2}-w^{4} \mathrm{P}_{01 / 2}^{01 / 2}\end{array}$ \\
\hline $\begin{array}{r}1 \\
5 \\
1 \\
5 \\
15\end{array}$ & $\begin{array}{l}1586.945 \\
1587.517 \\
1587.852 \\
1588.797 \\
1589.645\end{array}$ & $\begin{array}{l}63014.2 \\
62991.5 \\
62978.2 \\
62940.7 \\
62907.1\end{array}$ & $\frac{a{ }^{4} \mathrm{~F}_{21 / 2}-y{ }^{4} \mathrm{P}_{11 / 2}^{o}}{a^{6} \mathrm{D}_{31 / 2}-w^{4} \mathrm{~F}_{31 / 2}^{o}}$ & $\begin{array}{r}5 \\
15 \\
5 \\
15 \\
30\end{array}$ & $\begin{array}{l}\text { 1628. } 986 \\
\text { 1629. } 092 \\
1629.721 \\
1631.284 \\
1632.321\end{array}$ & $\begin{array}{l}61387.9 \\
61383.9 \\
61360.2 \\
61301.4 \\
61262.5\end{array}$ & $\begin{array}{c}a{ }^{2} \mathrm{G}_{41 / 2}-x{ }^{2} \mathrm{~F}_{31 / 2}^{\circ} \\
a{ }^{4} \mathrm{~F}_{31 / 2}-z \\
a^{4}{ }^{4} \mathrm{H}_{11 / 2}-y{ }^{4} \mathrm{~F}_{21 / 2}^{\circ} \\
a^{2}{ }^{2} \mathrm{H}_{51 / 2}-y{ }^{2} \mathrm{I}_{51 / 2}^{\circ}\end{array}$ \\
\hline $\begin{array}{l}30 \\
30 \\
15 \\
40 \\
30\end{array}$ & $\begin{array}{l}\text { 1590. } 774 \\
\text { 1590. } 882 \\
\text { 1591. } 744 \\
\text { 1593. } 403 \\
1594.398\end{array}$ & $\begin{array}{l}62862.5 \\
62858.2 \\
62824.2 \\
62758.8 \\
62719.6\end{array}$ & 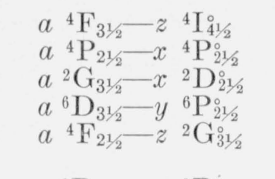 & $\begin{array}{r}10 \\
1 \\
30 \\
50 \\
100\end{array}$ & $\begin{array}{l}\text { 1633. } 802 \\
1634.306 \\
1635.129 \\
1635.333 \\
1635.948\end{array}$ & $\begin{array}{l}61206.9 \\
61188.1 \\
61157.3 \\
61149.6 \\
61126.6\end{array}$ & $\begin{array}{l}a^{4} \mathrm{D}_{21 / 2}-w^{4} \mathrm{P}_{21 / 2}^{0} \\
a^{6} \mathrm{D}_{01 / 2}-y \\
a^{2} \mathrm{G}_{41 / 2} \mathrm{P}_{11 / 2}^{0}-{ }^{2} \mathrm{~F}_{31 / 2}^{0} \\
a^{2} \mathrm{H}_{51 / 2}^{0} y{ }^{2} \mathrm{I}_{61 / 2}^{0}\end{array}$ \\
\hline $\begin{array}{r}30 \\
10 \\
1 \\
0 \\
20\end{array}$ & $\begin{array}{l}\text { 1594. } 603 \\
\text { 1596. } 482 \\
\text { 1597. } 172 \\
\text { 1597. } 890 \\
1598.491\end{array}$ & $\begin{array}{l}62711.5 \\
62637.7 \\
62610.7 \\
62582.5 \\
62559.0\end{array}$ & $\begin{array}{c}a{ }^{2} \mathrm{D}_{21 / 2}-y \\
a{ }^{4} \mathrm{P}_{11 / 2} \mathrm{P}^{0}-x{ }^{4} \mathrm{P}_{21 / 2}^{0} \\
a{ }^{4} \mathrm{P}_{11 / 2}-z{ }^{2} \mathrm{~S}_{01 / 2}^{0}\end{array}$ & $\begin{array}{l}30 \\
40 \\
20 \\
15 \\
20\end{array}$ & $\begin{array}{l}\text { 1637. } 446 \\
\text { 1637. } 591 \\
\text { 1638. } 366 \\
1639.209 \\
\text { 1639. } 371\end{array}$ & $\begin{array}{l}61070.7 \\
61065.3 \\
61036.4 \\
61005.0 \\
60999.0\end{array}$ & $\begin{array}{l}a{ }^{2} \mathrm{G}_{31 / 2}-x{ }^{2} \mathrm{H}_{41 / 2}^{\circ} \\
a^{2} \mathrm{G}_{41 / 2}-y{ }^{2} \mathrm{H}_{41 / 2}^{0} \\
-a{ }^{4} \mathrm{D}_{01 / 2}-w^{4} \mathrm{P}_{01 / 2}^{\circ} \\
a^{2} \mathrm{H}_{51 / 2}-w^{2} \mathrm{H}_{51 / 2}^{\circ}\end{array}$ \\
\hline $\begin{array}{r}30 \\
100 \\
1\end{array}$ & $\begin{array}{l}\text { 1599. } 937 \\
1600.183 \\
1602.095 \\
1602.447\end{array}$ & $\begin{array}{l}62492.9 \\
62418.3 \\
62404.6\end{array}$ & 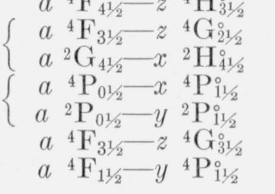 & $\begin{array}{r}15 \\
5 \\
2 \\
100 \\
10\end{array}$ & $\begin{array}{l}1639.530 \\
1639.963 \\
1640.756 \\
1641.460 \\
1643.312\end{array}$ & $\begin{array}{l}60993.1 \\
60977.0 \\
60947.5 \\
60921.4 \\
60852.7\end{array}$ & $\begin{array}{l}a{ }^{4} \mathrm{D}_{11 / 2}-w^{4} \mathrm{P}_{11 / 2}^{\circ} \\
a^{{ }^{4} \mathrm{~F}_{11 / 2}-z^{4} \mathrm{G}_{21 / 2}^{\circ}} \\
a^{2} \mathrm{H}_{41 / 2}-w^{2} \mathbf{F}_{31 / 2}^{0}\end{array}$ \\
\hline $\begin{array}{r}15 \\
10 \\
3 \\
30 \\
100\end{array}$ & $\begin{array}{l}\text { 1602. } 653 \\
1603.572 \\
1603.819 \\
1604.029 \\
1604.405\end{array}$ & $\begin{array}{l}62396.5 \\
62360.8 \\
62351.2 \\
62343.0 \\
62328.4\end{array}$ & 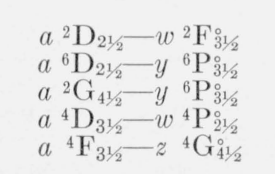 & $\begin{array}{r}2 \\
15 \\
10 \\
20 \\
5\end{array}$ & $\begin{array}{l}\text { 1643. } 434 \\
1644.914 \\
1645.706 \\
1647.615 \\
1647.913\end{array}$ & $\begin{array}{l}60848.2 \\
60793.5 \\
60764.2 \\
60693.8 \\
60682.8\end{array}$ & $\begin{array}{l}a^{2} \mathrm{G}_{31 / 2}-w^{4} \mathrm{~F}_{31 / 2}^{0} \\
a^{2} \mathrm{P}_{11 / 2}-w^{4} \mathrm{D}_{01 / 2}^{0} \\
a^{4} \mathrm{~F}_{11 / 2}-y{ }^{4} \mathrm{P}_{21 / 2}^{0} \\
a^{2} \mathrm{H}_{51 / 2}-w^{2} \mathrm{G}_{41 / 2}^{0}\end{array}$ \\
\hline $\begin{array}{r}1 \\
20 \\
15 \\
30 \\
5\end{array}$ & $\begin{array}{l}\text { 1604. } 767 \\
1605.111 \\
\text { 1605. } 616 \\
1605.787 \\
1608.733\end{array}$ & $\begin{array}{l}62314.3 \\
62301.0 \\
62281.4 \\
62274.8 \\
62160.7\end{array}$ & 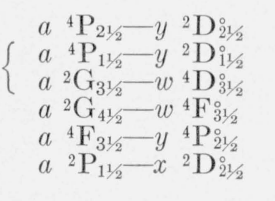 & $\begin{array}{r}15 \\
3 \\
1 \\
30 \\
1\end{array}$ & $\begin{array}{l}\text { 1648. } 069 \\
1650.327 \\
1650.659 \\
1651.898 \\
1652.363\end{array}$ & $\begin{array}{l}\text { 60677. } 1 \\
60594.1 \\
60581.9 \\
60536.4 \\
60519.4\end{array}$ & $\begin{array}{c}a^{2} \mathrm{G}_{31 / 2}-w^{4} \mathrm{~F}_{21 / 2}^{0} \\
a^{4} \mathrm{D}_{01 / 2}-w^{4} \mathrm{P}_{11 / 2}^{0} \\
a^{2} \mathrm{D}_{21 / 2}-x^{2} \mathrm{D}_{21 / 2}^{\circ} \\
a^{4} \mathrm{D}_{11}-w^{4} \mathrm{P}_{211}^{\circ}\end{array}$ \\
\hline $\begin{array}{r}10 \\
10 \\
50 \\
1 \\
3\end{array}$ & $\begin{array}{l}\text { 1610. } 372 \\
\text { 1610. } 464 \\
1611.415 \\
1613.158 \\
1613.685\end{array}$ & $\begin{array}{l}62097.5 \\
62093.9 \\
62057.3 \\
61990.2 \\
61969.9\end{array}$ & $\begin{array}{lll}a & { }^{2} \mathrm{D}_{11 / 2}-v & { }^{2} \mathrm{~F}_{21 / 2}^{\circ} \\
a & { }^{4} \mathrm{P}_{11 / 2}-y & { }^{2} \mathrm{D}_{21 / 2}^{21 / 2} \\
a & { }^{6} \mathrm{D}_{21 / 2}-y & { }^{6} \mathrm{P}_{21 / 2}^{0}\end{array}$ & $\begin{array}{r}2 \\
3 \\
1 \\
30 \\
1\end{array}$ & $\begin{array}{l}\text { 1653. } 564 \\
\text { 1654. } 477 \\
1656.747 \\
1657.217 \\
1657.617\end{array}$ & $\begin{array}{l}60475.4 \\
60442.1 \\
60359.2 \\
60342.1 \\
60327.6\end{array}$ & $\begin{array}{l}a^{4} \mathrm{D}_{11 / 2}-w^{4} \mathrm{P}_{21 / 2}^{2} \\
a{ }^{2} \mathrm{P}_{11 / 2}-w^{4} \mathrm{D}_{21 / 2}^{\circ} \\
a^{2} \mathrm{G}_{41 / 2}-y{ }^{2} \mathrm{H}_{51 / 2}^{\circ} \\
a^{2} \mathrm{P}_{11 / 2}-w^{4} \mathrm{D}_{21 / 2}^{2}\end{array}$ \\
\hline $\begin{array}{r}20 \\
20 \\
1 \\
15 \\
1\end{array}$ & $\begin{array}{l}\text { 1615. } 064 \\
\text { 1615. } 267 \\
\text { 1615. } 656 \\
\text { 1617. } 663 \\
\text { 1618. } 781\end{array}$ & $\begin{array}{l}\text { 61917. } 1 \\
61909.3 \\
61894.4 \\
61817.6 \\
61774.9\end{array}$ & $\begin{array}{l}a^{6} \mathrm{D}_{21 / 2}-y^{6} \mathrm{P}_{11 / 2}{ }^{1 / 2} \\
a^{4} \mathrm{P}_{21 / 2}-1_{11 / 2}^{1}{ }^{\circ} \\
a^{4} \mathrm{~F}_{11 / 2}-y{ }^{4} \mathrm{~F}_{11 / 2}\end{array}$ & $\begin{array}{r}10 \\
100 \\
0 \\
2 \\
0\end{array}$ & $\begin{array}{l}\text { 1658. } 457 \\
\text { 1659. } 364 \\
\text { 1659. } 871 \\
\text { 1660. } 059 \\
1662.684\end{array}$ & $\begin{array}{l}\text { 60297. } 0 \\
60264.1 \\
60245.6 \\
60238.8 \\
60143.7\end{array}$ & 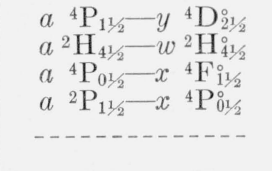 \\
\hline $\begin{array}{r}1 \\
20 \\
20 \\
30 \\
5\end{array}$ & $\begin{array}{l}\text { 1618. } 986 \\
\text { 1619. } 365 \\
\text { 1620. } 102 \\
\text { 1620. } 865 \\
\text { 1621. } 445\end{array}$ & $\begin{array}{l}61767.1 \\
61752.6 \\
61724.5 \\
61695.5 \\
61673.4\end{array}$ & $\begin{array}{l}a^{2} \mathrm{G}_{31 / 2}-x^{2} \mathrm{G}_{11 / 2}^{\circ} \\
a^{4} \mathrm{D}_{21 / 2}-w^{4} \mathrm{P}_{11 / 2}^{1} \\
a^{4} \mathrm{P}_{11 / 2}-1_{11 / 2}^{\circ}\end{array}$ & $\begin{array}{c}10 e h \\
2 \\
3 \\
3 \\
5\end{array}$ & $\begin{array}{l}\text { 1666. } 312 \\
\text { 1667. } 426 \\
1667.912 \\
1670.788 \\
1671.412\end{array}$ & $\begin{array}{l}60012.8 \\
59972.7 \\
59955.2 \\
59852.0 \\
59829.7\end{array}$ & $\left\{\begin{array}{l}a{ }^{2} \mathrm{P}_{11 / 2}-w^{4} \mathrm{~F}_{21 / 2}^{\circ} \\
a^{2} \mathrm{D}_{21 / 2}-w^{4} \mathrm{D}_{31 / 2}^{\circ} \\
a^{4} \mathrm{P}_{21 / 3}-x \\
a^{4} \mathrm{D}_{31 / 2}^{\circ} \mathrm{G}_{31 / 2}-x \\
{ }^{2} \mathrm{~F}_{31 / 2}^{\circ} \\
a^{2} \mathrm{P}_{11 / 2}-2_{11 / 2}^{\circ}\end{array}\right.$ \\
\hline
\end{tabular}


TABLE 6. The second spectrum of ruthenium (Ru II) - Continued

\begin{tabular}{|c|c|c|c|c|c|c|c|}
\hline $\begin{array}{c}1 \\
\text { Intensity }\end{array}$ & $\begin{array}{c}2 \\
\lambda(\text { vacuum })\end{array}$ & $\begin{array}{l}3 \\
\sigma\end{array}$ & $\begin{array}{c}4 \\
\text { Term combination }\end{array}$ & $\begin{array}{c}1 \\
\text { Intensity }\end{array}$ & $\begin{array}{c}2 \\
\lambda(\text { vacuum })\end{array}$ & $\begin{array}{l}3 \\
\sigma\end{array}$ & $\begin{array}{c}4 \\
\text { Term combination }\end{array}$ \\
\hline $\begin{array}{r}0 \\
30 \\
5 \\
3 \\
2\end{array}$ & $\begin{array}{c}A \\
\text { 1671. } 819 \\
1672.225 \\
1672.445 \\
1676.922 \\
1678.781\end{array}$ & $\begin{array}{c}K \\
59815.1 \\
59800.6 \\
59792.7 \\
59633.1 \\
59567.0\end{array}$ & $\begin{array}{l}a^{2} \mathrm{H}_{41 / 2}-y^{2} \mathrm{I}_{51 / 2}^{\circ} \\
a^{2} \mathrm{P}_{01 / 2}-x{ }^{2} \mathrm{D}_{11 / 2}^{1} \\
a^{2} \mathrm{G}_{31 / 2}-y{ }^{2} \mathrm{H}_{41 / 2}^{\circ} \\
a^{2} \mathrm{P}_{11 / 2}-w^{4} \mathrm{~F}_{11 / 2}^{0} ?\end{array}$ & $\begin{array}{l}0 \\
3 \\
2 \\
1 \\
1\end{array}$ & $\begin{array}{c}A \\
1748.441 \\
1750.239 \\
1750.979 \\
1752.835 \\
1753.173\end{array}$ & $\begin{array}{c}K \\
57193.8 \\
57135.1 \\
57110.9 \\
57050.4 \\
57039.4\end{array}$ & $\begin{array}{l}a{ }^{2} \mathrm{P}_{11 / 2}-1_{11 / 2}^{\circ} \\
a^{2} \mathrm{H}_{51 / 2}-z^{2} \mathrm{~K}_{61 / 2}^{\circ} \\
a^{2} \mathrm{G}_{31 / 2}-x{ }^{4} \mathrm{~F}_{31 / 2}^{\circ 1 / 2} \\
a^{2} \mathrm{G}_{41 / 2}-x^{4} \mathrm{~F}_{41 / 2}^{\circ}\end{array}$ \\
\hline $\begin{array}{c}30 \\
1 \\
50 \\
1 \\
15 e\end{array}$ & $\begin{array}{l}\text { 1679. } 620 \\
1683.810 \\
1683.985 \\
1684.311 \\
1686.161\end{array}$ & $\begin{array}{l}59537.3 \\
59389.1 \\
59383.0 \\
59371.5 \\
59306.3\end{array}$ & $\begin{array}{c}a^{2} \mathrm{H}_{41 / 2}-w^{2} \mathrm{H}_{51 / 2}^{\circ} \\
a^{4} \mathrm{P}_{21 / 2}-y{ }^{4} \mathrm{G}_{31 / 2} \\
a^{2} \mathrm{H}_{51 / 2}-x{ }^{2} \mathrm{G}_{41 / 2}^{0} \\
a^{2} \mathrm{G}_{31 / 2}-y^{2} \mathrm{~F}_{21 / 2}^{0}\end{array}$ & $\begin{array}{r}30 \\
5 \\
5 \\
10 \\
3\end{array}$ & $\begin{array}{l}1753.441 \\
1754.756 \\
1755.728 \\
1757.854 \\
1759.769\end{array}$ & $\begin{array}{l}57030.7 \\
56988.0 \\
56956.4 \\
56887.5 \\
56825.6\end{array}$ & $\begin{array}{lll}a & { }^{4} \mathrm{P}_{11 / 2}-y & { }^{4} \mathrm{P}_{11 / 2}^{0} \\
a & { }^{4} \mathrm{P}_{21 / 2}-z & 2 \\
a^{4} \mathrm{D}_{21 / 2}^{0} \\
{ }^{4} \mathrm{P}_{21 / 2}-z & { }^{2} \mathrm{G}_{1 / 2}^{0} \\
a^{2} & { }^{2} \mathrm{G}_{31 / 2}-y & { }^{2} \mathrm{G}_{41 / 2}^{0} \\
a^{2} & { }^{2} \mathrm{~F}_{31 / 2}-v & { }^{2} \mathrm{~F}_{21 / 2}^{0}\end{array}$ \\
\hline $\begin{array}{r}100 \\
10 \\
1 \\
2 \\
20\end{array}$ & $\begin{array}{l}\text { 1686. } 691 \\
1688.357 \\
1692.828 \\
1694.677 \\
1697.943\end{array}$ & $\begin{array}{l}59287.7 \\
59229.2 \\
59072.7 \\
59008.3 \\
58894.8\end{array}$ & 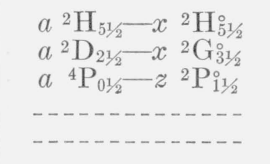 & $\begin{array}{r}20 \\
15 \\
1 \\
0 \\
20\end{array}$ & $\begin{array}{l}1761.030 \\
1761.580 \\
1763.996 \\
1764.829 \\
1765.525\end{array}$ & $\begin{array}{l}56784.9 \\
56767.2 \\
56689.5 \\
56662.7 \\
56640.4\end{array}$ & 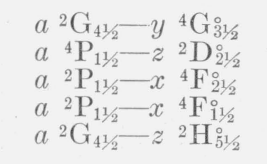 \\
\hline $\begin{array}{r}0 \\
20 \\
3 \\
1 \\
30\end{array}$ & $\begin{array}{l}\text { 1699. } 330 \\
1699.442 \\
1700.049 \\
1700.839 \\
1701.080\end{array}$ & $\begin{array}{l}58846.7 \\
58842.8 \\
58821.8 \\
58794.5 \\
58786.2\end{array}$ & $\left\{\begin{array}{l}a^{4} \mathrm{P}_{21 / 2}-x{ }^{4} \mathrm{D}_{11 / 2}^{\circ} \\
a^{6} \mathrm{D}_{11 / 2}-1_{11 / 2}^{\circ} \\
a^{4} \mathrm{P}_{21 / 2}-z{ }^{2} \mathrm{~F}_{211 / 2}^{\circ} \\
a^{2} \mathrm{G}_{31 / 2}-x{ }^{4} \mathrm{P}_{21 / 2}^{\circ} \\
a^{2} \mathrm{G}_{41 / 2}-y{ }^{4} \mathrm{H}_{31 / 2}^{\circ} \\
a^{2} \mathrm{G}_{41 / 2}-y{ }^{4} \mathrm{H}_{51 / 2}^{\circ}\end{array}\right.$ & $\begin{array}{r}20 \\
5 \\
15 \\
50 \\
5\end{array}$ & $\begin{array}{l}1768.666 \\
1770.160 \\
1770.446 \\
1776.061 \\
1777.432\end{array}$ & $\begin{array}{l}56539.8 \\
56492.1 \\
56482.9 \\
56304.4 \\
56260.9\end{array}$ & 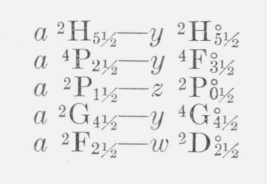 \\
\hline $\begin{array}{r}3 \\
1 \\
50 \\
10 \\
5\end{array}$ & $\begin{array}{l}\text { 1702. } 061 \\
1702.572 \\
1703.562 \\
1703.818 \\
1705.263\end{array}$ & $\begin{array}{l}58752.3 \\
58734.7 \\
58700.5 \\
58691.7 \\
58642.0\end{array}$ & $\begin{array}{l}a^{2} \mathrm{G}_{41 / 2}-y{ }^{4} \mathrm{H}_{41 / 2}^{\circ} \\
a^{2} \mathrm{D}_{21 / 2}-w^{4} \mathrm{D}_{11 / 2}^{\circ} \\
a^{2} \mathrm{H}_{51 / 2}-x \\
a^{2} \mathrm{H}_{41 / 2}^{\circ} \mathrm{G}_{41 / 2}-x \\
a^{4} \mathrm{G}_{31 / 2}^{\circ} \\
a^{2} \mathrm{P}_{11 / 2}-y{ }^{2} \mathrm{~F}_{21 / 2}^{\circ}\end{array}$ & $\begin{array}{r}10 \\
5 \\
0 \\
0 \\
0\end{array}$ & $\begin{array}{l}\text { 1778. } 206 \\
1779.124 \\
1779.420 \\
1780.057 \\
1781.407\end{array}$ & $\begin{array}{l}56236.4 \\
56207.4 \\
56198.1 \\
56178.0 \\
56135.4\end{array}$ & $\begin{array}{lll}a & { }^{2} \mathrm{P}_{01 / 2}-z & { }^{2} \mathrm{~S}_{01 / 2}^{\circ} \\
a & { }^{4} \mathrm{P}_{21 / 2}-y & { }^{4} \mathrm{~F}_{21 / 2}^{\circ} \\
a^{2} \mathrm{D}_{21 / 2}-y & { }^{2} \mathrm{D}_{11 / 2}^{\circ} \\
a & { }^{2} \mathrm{D}_{11 / 2}-x & { }^{4} \mathrm{P}_{01 / 2}^{\circ} \\
a & { }^{4} \mathrm{P}_{01 / 2}-y & { }^{4} \mathrm{P}_{11 / 2}^{\circ}\end{array}$ \\
\hline $\begin{array}{r}2 \\
1 \\
1 \\
10 \\
15\end{array}$ & $\begin{array}{l}1705.572 \\
1705.735 \\
1705.840 \\
1707.608 \\
1708.620\end{array}$ & $\begin{array}{l}58631.4 \\
58625.8 \\
58622.1 \\
58561.4 \\
58526.8\end{array}$ & $\begin{array}{l}a{ }^{2} \mathrm{D}_{21 / 2}-y{ }^{6} \mathrm{P}_{31 / 2}^{\circ} ? \\
a{ }^{4} \mathrm{P}_{11 / 2}-x \\
{ }^{4} \mathrm{D}_{11 / 2}^{\circ} \\
a{ }^{4} \mathrm{P}_{11 / 2}-z \\
a^{2} \mathrm{~F}_{21 / 2}^{\circ} \\
{ }^{2} \mathrm{D}_{21 / 2}-w^{4} \mathrm{~F}_{31 / 2}^{\circ} \\
a^{4} \mathrm{P}_{01 / 2}-y{ }^{4} \mathrm{D}_{11 / 2}^{\circ}\end{array}$ & $\begin{array}{r}5 \\
0 \\
20 \\
1 \\
2\end{array}$ & $\begin{array}{l}\text { 1781. } 792 \\
\text { 1784. } 767 \\
1786.032 \\
\text { 1786. } 155 \\
1786.347\end{array}$ & $\begin{array}{l}56123.3 \\
56029.7 \\
55990.0 \\
55986.2 \\
55980.2\end{array}$ & 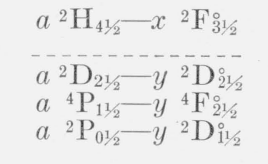 \\
\hline $\begin{array}{r}20 \\
20 \\
3 \\
3 \\
20\end{array}$ & $\begin{array}{l}\text { 1710. } 299 \\
1712.456 \\
1714.667 \\
1717.160 \\
1718.975\end{array}$ & $\begin{array}{l}58469.3 \\
58395.7 \\
58320.4 \\
58235.7 \\
58174.2\end{array}$ & $\begin{array}{c}a^{2} \mathrm{H}_{41 / 2}-w^{4} \mathrm{D}_{31 / 2}^{\circ} \\
a{ }^{2} \mathrm{P}_{01 / 2}-x \\
a^{4} \mathrm{P}_{01 / 2}^{0} \mathrm{G}_{41 / 2}-y \\
{ }^{2} \mathrm{G}_{41 / 2}^{0} \\
a^{4} \mathrm{P}_{11 / 2}-x{ }^{4} \mathrm{D}_{21 / 2}^{0}\end{array}$ & $\begin{array}{r}10 \\
1 \\
1 \\
20 \\
20\end{array}$ & $\begin{array}{l}\text { 1787. } 233 \\
1787.770 \\
1789.383 \\
1791.253 \\
1791.560\end{array}$ & $\begin{array}{l}55952.4 \\
55935.6 \\
55885.2 \\
55826.8 \\
55817.3\end{array}$ & 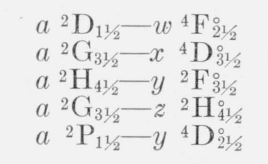 \\
\hline $\begin{array}{r}10 \\
15 \\
15 \\
0 \\
5\end{array}$ & $\begin{array}{l}\text { 1721. } 171 \\
1721.712 \\
1721.778 \\
1725.761 \\
1729.417\end{array}$ & $\begin{array}{l}58100.0 \\
58081.7 \\
58079.5 \\
57945.5 \\
57823.0\end{array}$ & 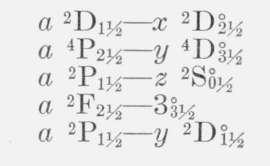 & $\begin{array}{r}2 \\
1 \\
10 \\
15 \\
15\end{array}$ & $\begin{array}{l}1792.072 \\
1797.118 \\
1799.549 \\
1800.470 \\
1806.599\end{array}$ & $\begin{array}{l}55801.3 \\
55644.6 \\
55569.5 \\
55541.1 \\
55352.6\end{array}$ & 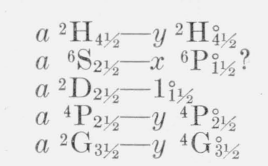 \\
\hline $\begin{array}{r}1 \\
50 \\
10 \\
20 \\
5\end{array}$ & $\begin{array}{l}1731.110 \\
1731.415 \\
1732.209 \\
1733.537 \\
1734.069\end{array}$ & $\begin{array}{l}57766.4 \\
57756.2 \\
57729.8 \\
57685.5 \\
57667.8\end{array}$ & $\begin{array}{l}a{ }^{4} \mathrm{P}_{21 / 2}-z{ }^{2} \mathrm{~F}_{31 / 2}^{\circ} \\
a{ }^{4} \mathrm{P}_{01 / 2}-x \\
{ }^{4} \mathrm{D}_{11 / 2}^{\circ} \\
a^{2} \mathrm{H}_{41 / 2}-x \\
a^{2} \mathrm{G}_{31 / 2}^{0} \mathrm{D}_{21 / 2}-x\end{array}$ & $\begin{array}{l}5 \\
5 \\
1 \\
5 \\
3\end{array}$ & $\begin{array}{l}\text { 1807. } 658 \\
1813.558 \\
1815.607 \\
1816.030 \\
1818.527\end{array}$ & $\begin{array}{l}55320.2 \\
55140.2 \\
55078.0 \\
55065.2 \\
54989.6\end{array}$ & 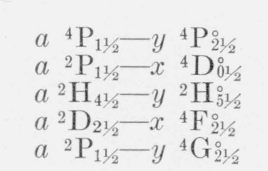 \\
\hline $\begin{array}{l}15 \\
10 \\
15 \\
15 \\
20\end{array}$ & $\begin{array}{l}1735.654 \\
1737.389 \\
1738.666 \\
1740.114 \\
1741.262\end{array}$ & $\begin{array}{l}57615.2 \\
57557.6 \\
57515.4 \\
57467.5 \\
57429.6\end{array}$ & $\begin{array}{lll}a & { }^{2} \mathrm{P}_{11 / 2}-y & { }^{2} \mathrm{D}_{21 / 2}^{\circ} \\
a & { }^{2} \mathrm{~F}_{21 / 2}-v & { }^{2} \mathrm{~F}_{21 / 2}^{2} \\
a & { }^{2} \mathrm{~F}_{31 / 2}-v & { }^{2} \mathrm{~F}_{31 / 2}^{0} \\
a & { }^{2} \mathrm{G}_{41 / 2}-x & { }^{4} \mathrm{G}_{51 / 2}^{\circ} \\
a^{2} \mathrm{D}_{21 / 2}-y & { }^{2} \mathrm{~F}_{31 / 2}^{\circ}\end{array}$ & $\begin{array}{r}20 \\
30 \\
3 \\
5 \\
2\end{array}$ & $\begin{array}{l}\text { 1818. } 724 \\
\text { 1819. } 125 \\
\text { 1819. } 852 \\
1820.059 \\
1820.999\end{array}$ & $\begin{array}{l}54983.6 \\
54971.5 \\
54949.5 \\
54943.3 \\
54914.9\end{array}$ & 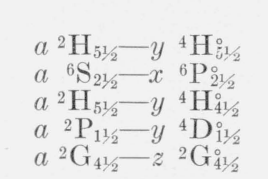 \\
\hline $\begin{array}{l}1 \\
5\end{array}$ & $\begin{array}{l}1743.514 \\
1744.614\end{array}$ & $\begin{array}{l}57355.4 \\
57319.3\end{array}$ & $a^{2} \mathrm{G}_{31 / 2}-y^{4} \mathrm{H}_{41 / 2}^{0}$ & 1 & 1822. 238 & 54877.6 & \\
\hline 30 & 1746. 446 & 57259. 1 & $\left\{\begin{array}{l}a^{2} \mathrm{G}_{41 / 2}-z{ }^{2} \mathrm{H}_{41 / 2}^{\circ} \\
a^{2} \mathrm{G}_{31 / 2}-x{ }^{4} \mathrm{G}_{31 / 2}^{0}\end{array}\right.$ & $\begin{array}{r}1 \\
10\end{array}$ & $\begin{array}{l}\text { 1822. } 427 \\
\text { 1824. } 619\end{array}$ & $\begin{array}{l}54871.9 \\
54806.0\end{array}$ & $\begin{array}{l}a^{2} \mathrm{G}_{31 / 2}-y{ }^{4} \mathrm{G}_{41 / 2}^{\circ} \\
a^{2} \mathrm{G}_{31 / 2}-z{ }^{2} \mathrm{~F}_{21 / 2}^{\circ}\end{array}$ \\
\hline $\begin{array}{l}3 \\
1\end{array}$ & $\begin{array}{l}\text { 1747. } 804 \\
1748.310\end{array}$ & $\begin{array}{l}57214.7 \\
57198.1\end{array}$ & $a^{2} \mathrm{~F}_{31 / 2}^{0 / 2}-3_{31 / 2}^{\circ}$ & $\begin{array}{l}0 \\
5\end{array}$ & $\begin{array}{l}1828.521 \\
1829.894\end{array}$ & $\begin{array}{l}54689.0 \\
54648.0\end{array}$ & $\begin{array}{l}a^{2} \mathrm{~F}_{31 / 2}-w^{2} \mathrm{~F}_{31 / 2}^{2 / 2} \\
a^{2} \mathrm{H}_{51 / 2}-y{ }^{4} \mathrm{H}_{61 / 2}^{\circ}\end{array}$ \\
\hline
\end{tabular}


TABLE 6. The second spectrum of ruthenium (Ru II)-Continued

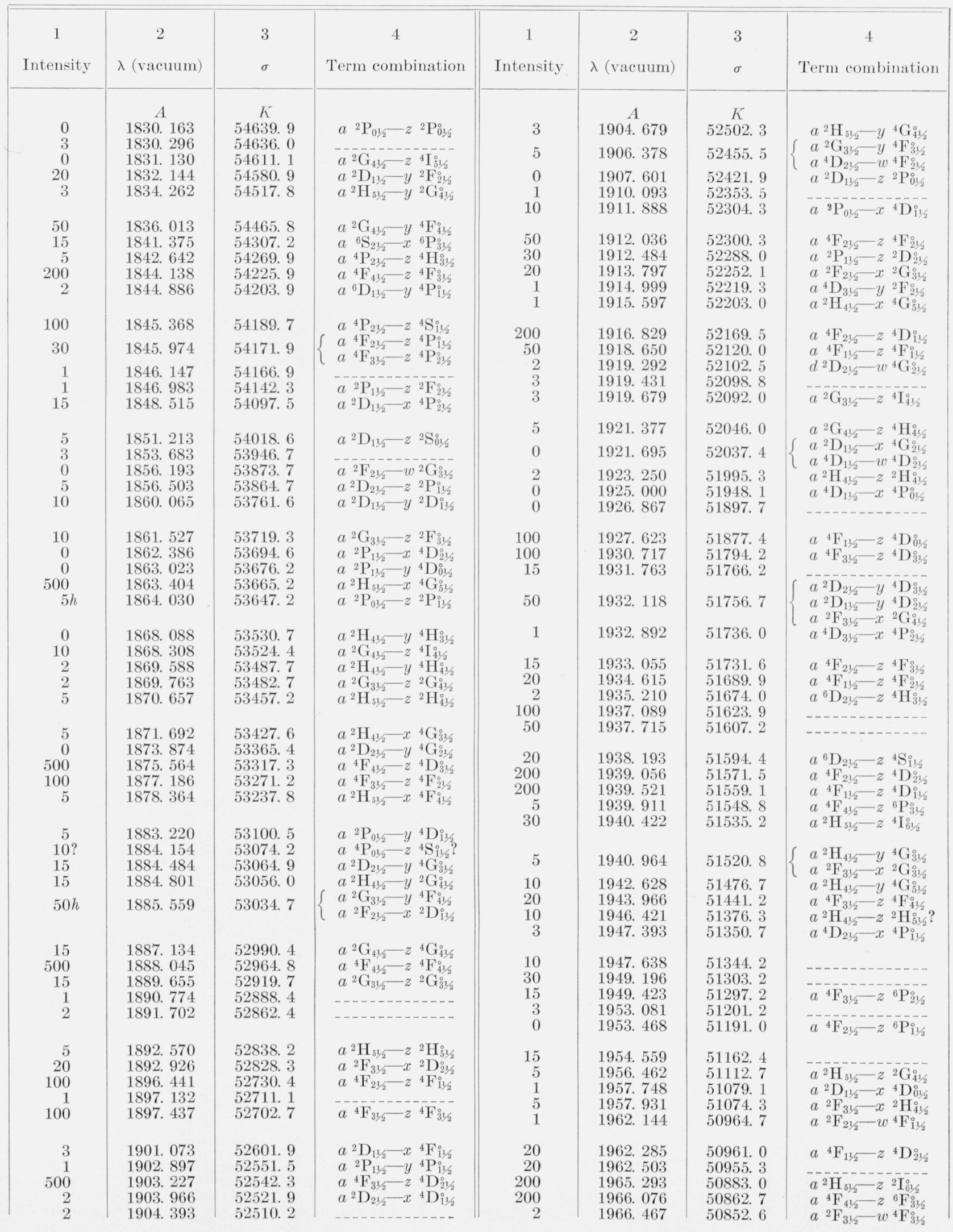


TABLE 6. The second spectrum of ruthenium (Ru II)-Continued

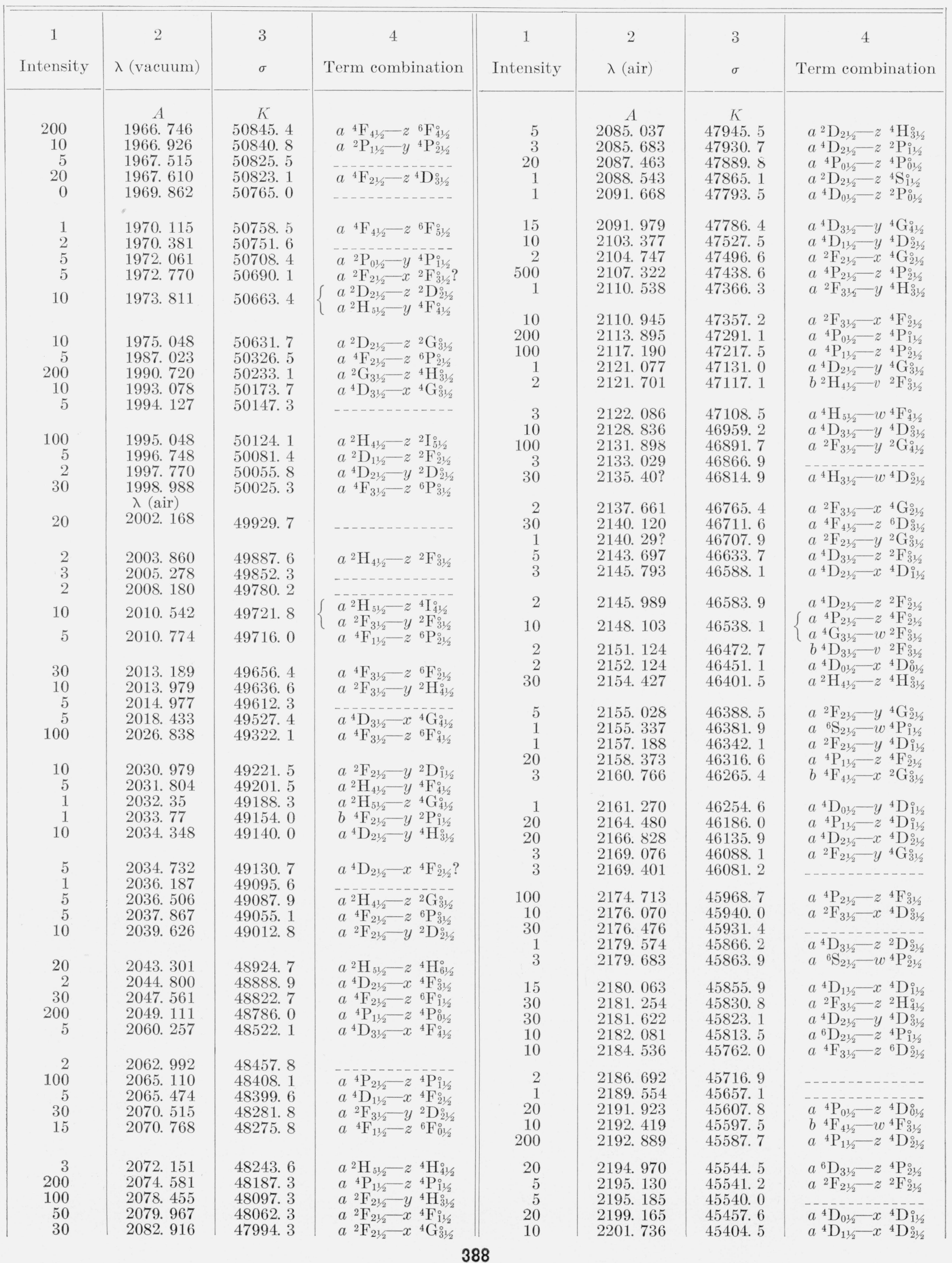


TABLE 6. The second spectrum of ruthenium (Ru II)-Continued

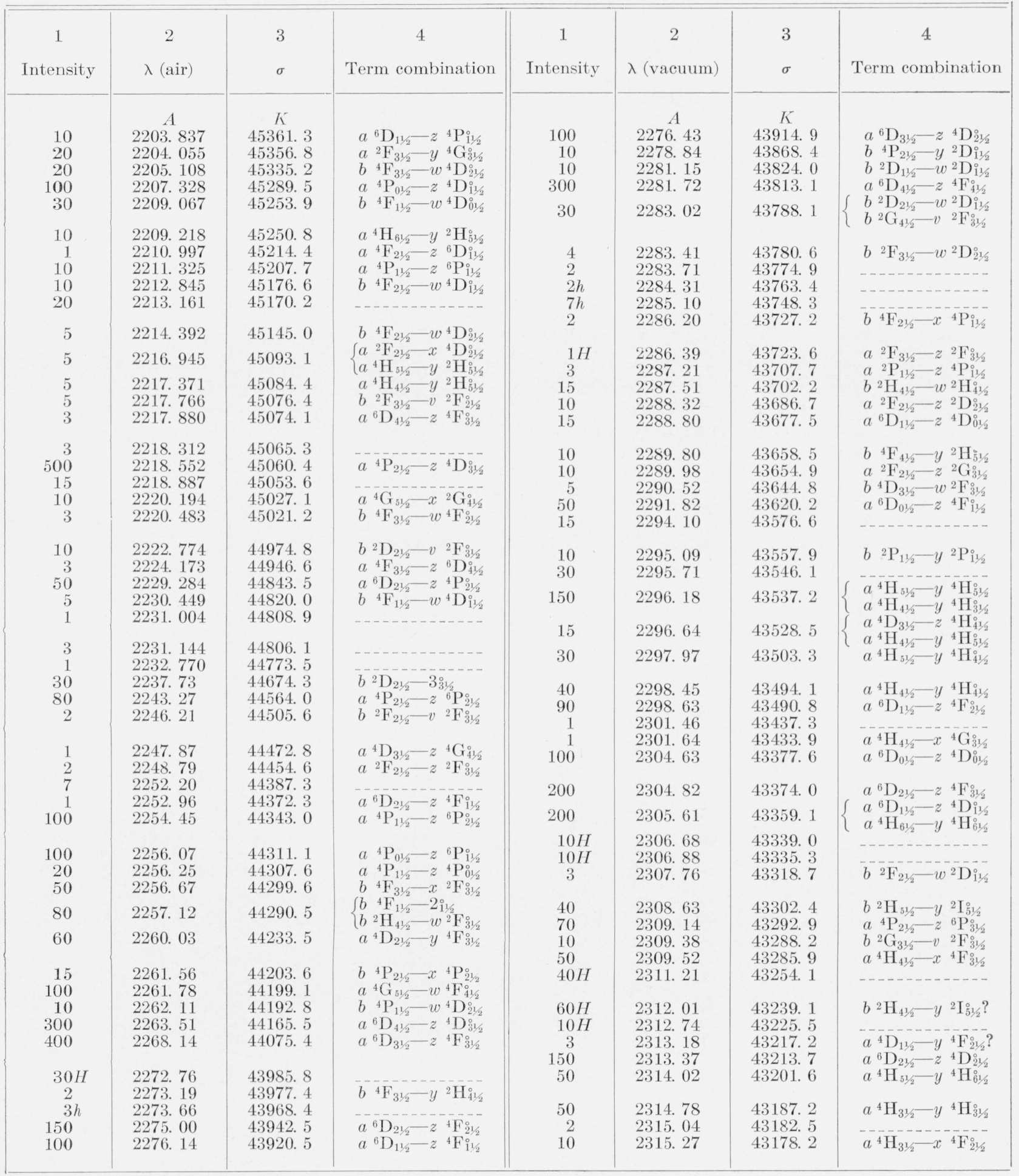


TABLE 6. The second spectrum of ruthenium (Ru II)-Continued

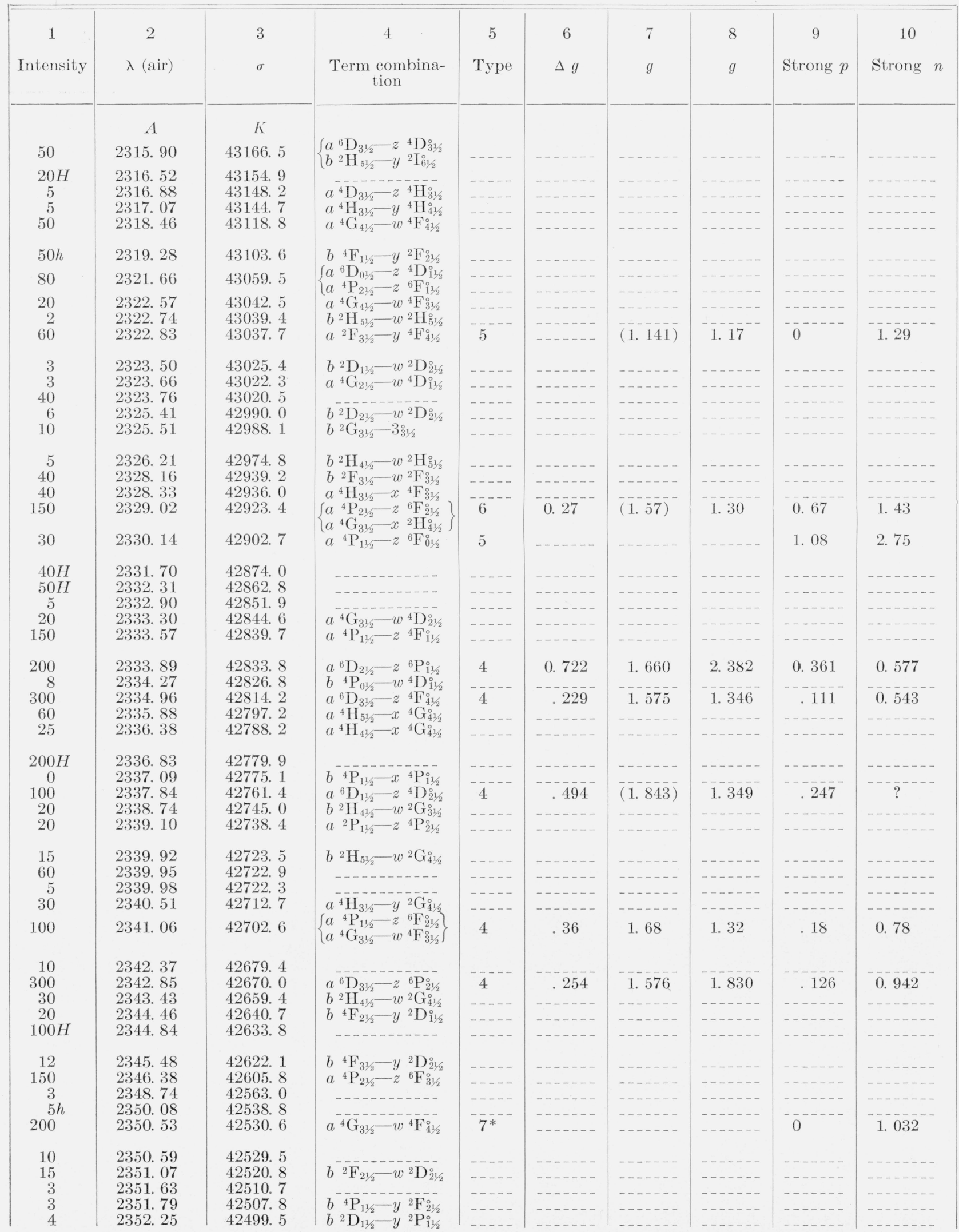


TABLE 6. The second spectrum of ruthenium (Ru II)-Continued

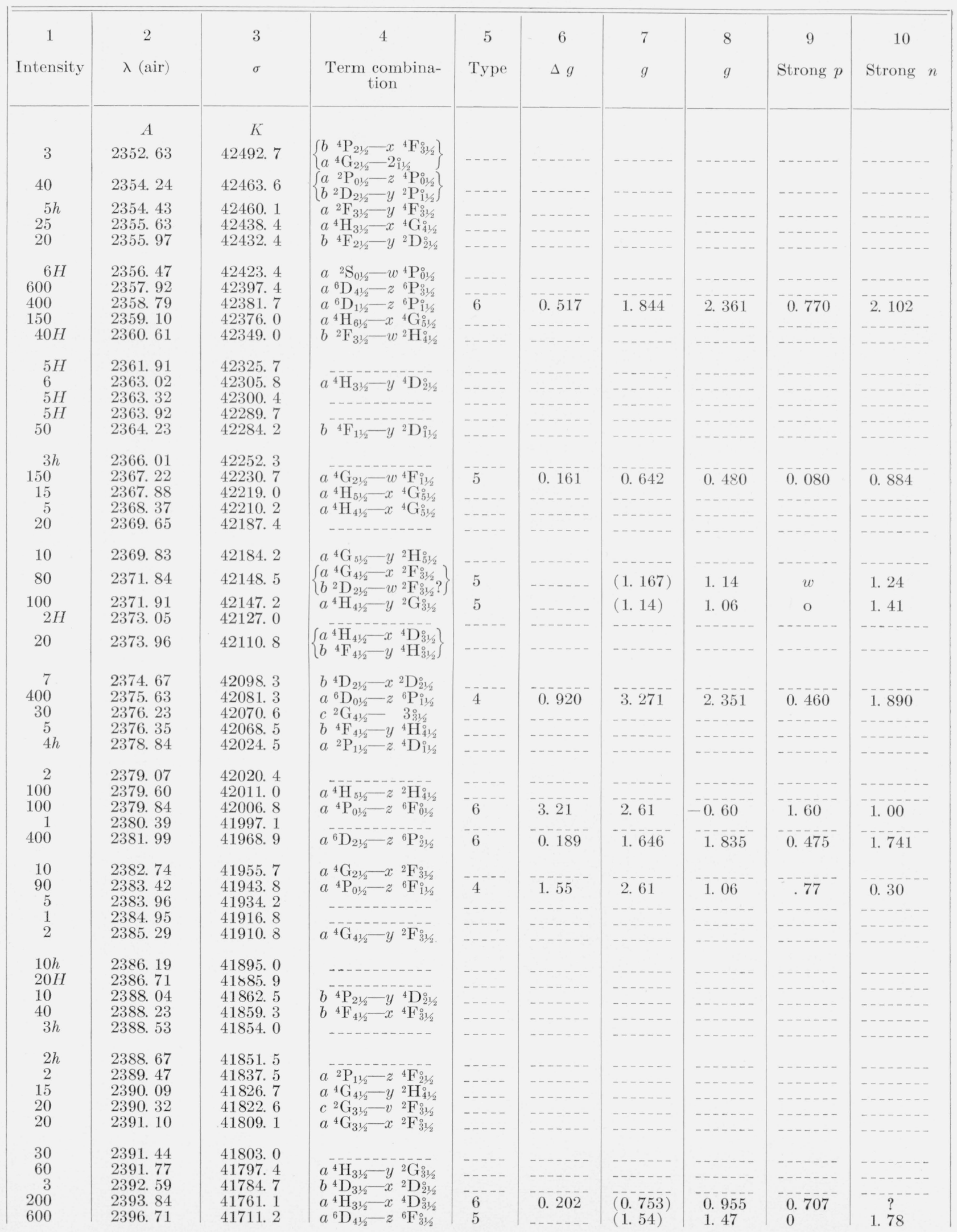


TABLE 6. The second spectrum of ruthenium (Ru II)-Continued

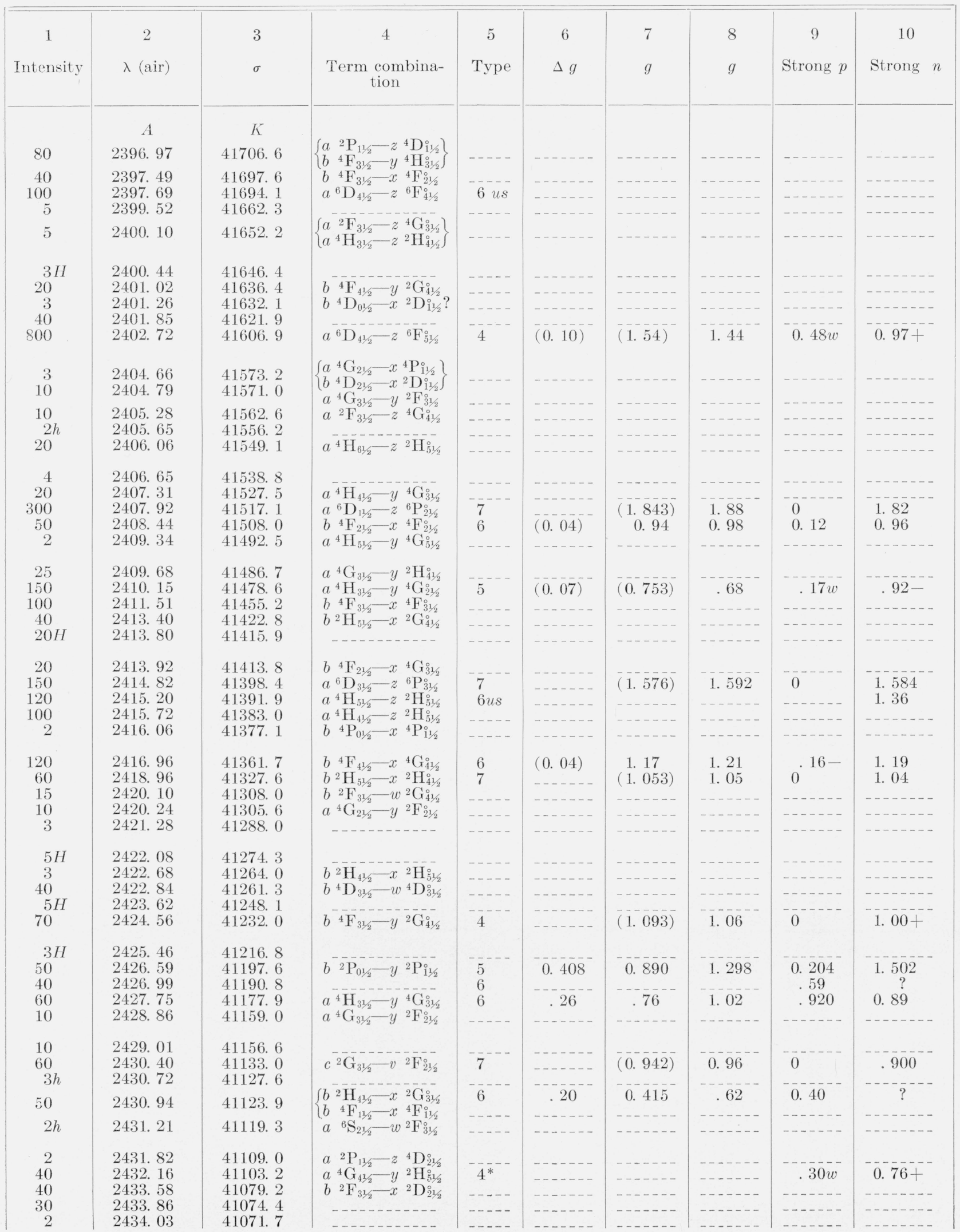


TABLE 6. The second spectrum of ruthenium (Ru II) - Continued

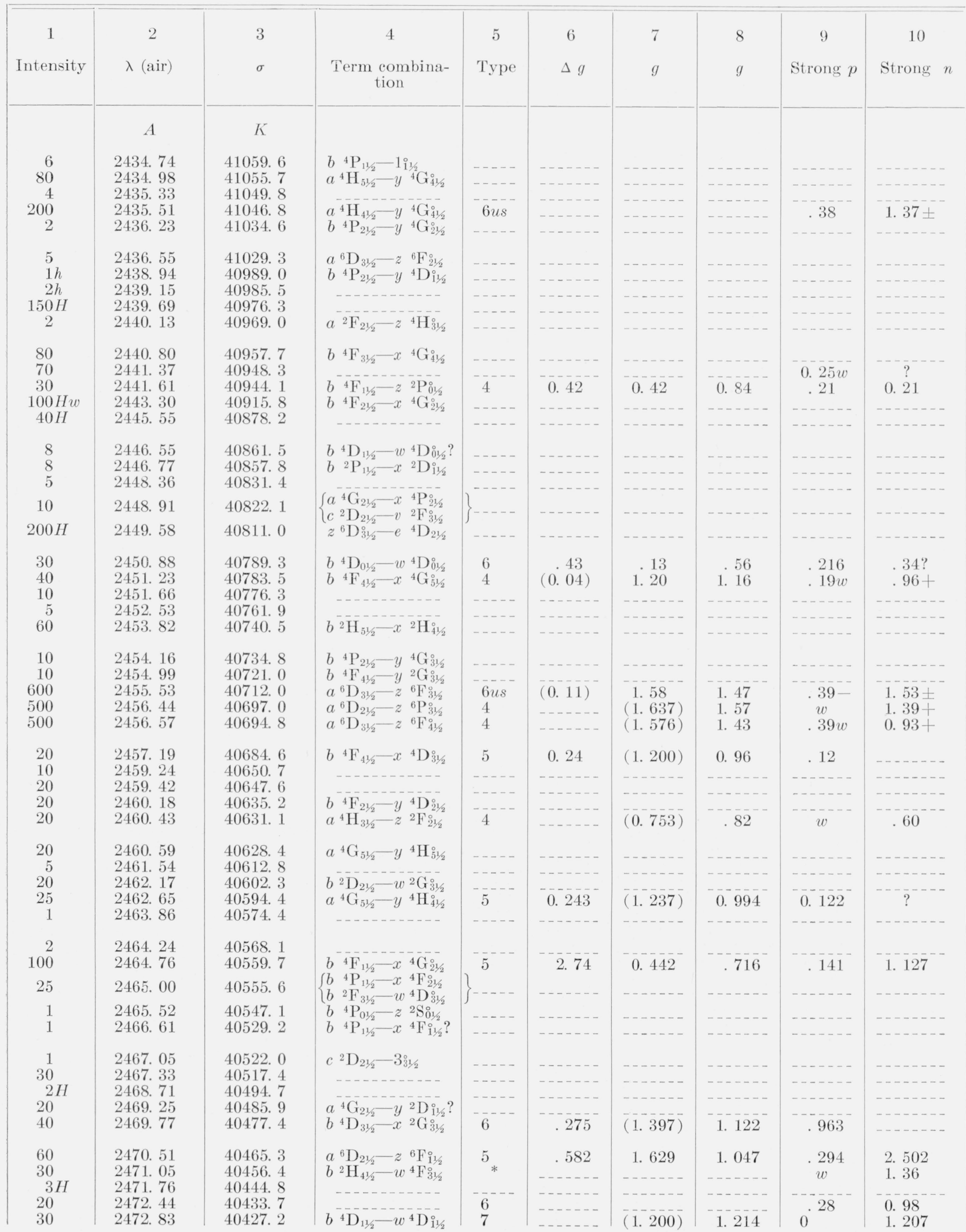


TABLE 6. The second spectrum of ruthenium (Ru II) - Continued

\begin{tabular}{|c|c|c|c|c|c|c|c|c|c|}
\hline $\begin{array}{c}1 \\
\text { Intensity }\end{array}$ & $\begin{array}{c}2 \\
\lambda(\text { air })\end{array}$ & $\begin{array}{l}3 \\
\sigma\end{array}$ & $\begin{array}{c}4 \\
\text { Term combina- } \\
\text { tion }\end{array}$ & $\begin{array}{c}5 \\
\text { Type }\end{array}$ & $\begin{array}{c}6 \\
\Delta g\end{array}$ & $\begin{array}{l}7 \\
g\end{array}$ & $\begin{array}{l}8 \\
g\end{array}$ & $\begin{array}{c}9 \\
\text { Strong } p\end{array}$ & $\begin{array}{c}10 \\
\text { Strong } n\end{array}$ \\
\hline & $A$ & $K$ & & & & & & & \\
\hline 8 & 2472. 99 & 40424.7 & $a{ }^{2} \mathrm{P}_{01 / 2}-z{ }^{4} \mathrm{~F}_{11 / 2}^{0}$ & & $\ldots \ldots-$ & $\ldots \ldots$ & $\ldots \ldots$ & $\ldots \ldots$ & $-\ldots-\ldots$ \\
\hline 10 & 2473. 57 & 40415. 1 & $\ldots \ldots$ & ---- & $\ldots \ldots$ & $\ldots \ldots$ & $\ldots \ldots$ & $\ldots \ldots$ & $\ldots \ldots \ldots$ \\
\hline $\begin{array}{l}5 h \\
30\end{array}$ & $\begin{array}{l}2473.83 \\
2474.76\end{array}$ & $\begin{array}{l}40410.9 \\
40395.7\end{array}$ & $b^{4} \mathrm{D}_{11 / 2}-w^{4} \mathrm{D}_{21 / 2}^{\circ}$ & & 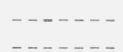 & $(1.200)$ & 1.30 & $w^{-\cdots}$ & 1.44 \\
\hline 4 & 2476.15 & 40373. 1 & b ${ }^{2} \mathrm{G}_{41 / 2}-w^{2} \mathrm{H}_{41 / 2}^{0+2}$ & $\ldots$ & - n- & (1.2. & - 1.200 & $\ldots+\ldots$ & 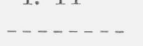 \\
\hline 10 & 2477.17 & 40356. 4 & $b{ }^{4} \mathrm{~F}_{41 / 2}-x{ }^{4} \mathrm{~F}_{41 / 2}^{\circ}$ & . . $\ldots$ & $\ldots \ldots \ldots$ & $\ldots \ldots \ldots$ & $\ldots \ldots$ & $\ldots \ldots$ & _....... \\
\hline 50 & 2477. 26 & 40355. 0 & $b{ }^{4} \mathrm{D}_{01 / 2}-w^{4} \mathrm{D}_{11 / 2}$ & $-\ldots$ & $\ldots \ldots$ & - n & $\ldots \ldots$ & $\ldots \ldots$ & - \\
\hline 8 & 2477. 98 & 40343. 3 & $\ldots \ldots \ldots$ & ---- & $\ldots \ldots$ & $\ldots-\ldots$ & $\ldots \ldots$ & $\ldots \ldots-$ & $\ldots-\ldots$ \\
\hline $\begin{array}{r}20 \\
500\end{array}$ & $\begin{array}{l}2478.36 \\
2478.93\end{array}$ & $\begin{array}{l}\text { 40337. } 2 \\
40327.9\end{array}$ & $a^{6} \mathrm{D}_{21 / 2}-z^{6} \mathrm{~F}_{21 / 2}^{\circ}$ & --- & 0.334 & 1.635 & 1.301 & 0.851 & 1. 468 \\
\hline 5 & 2480.20 & 40307. 0 & $\left\{\begin{array}{lll}b & { }^{4} \mathrm{~F}_{21 / 2}-z & { }^{2} \mathrm{P}_{11 / 2}^{\circ} \\
b & { }^{4} \mathrm{D}_{11 / 2}-x & { }^{4} \mathrm{P}_{01 / 2}^{\circ}\end{array}\right.$ & - & - . - n & $-\cdots--$ & $\ldots-\cdots$ & $\ldots \ldots$ & $-\ldots-\ldots$ \\
\hline 30 & 2480.81 & 40297. 1 & $a^{4} \mathrm{H}_{61 / 2}-z^{2} \mathrm{I}_{51 / 2}^{\circ}$ & 5 & .181 & (1. 23$)$ & 1. 05 & .09 & 2. $16 ?$ \\
\hline 200 & 2481. 11 & $\begin{array}{l}40292.4 \\
40280.4\end{array}$ & $\begin{array}{l}a{ }^{4} \mathrm{G}_{51 / 2}-y{ }^{4} \mathrm{H}_{61 / 2}^{\circ} \\
b{ }^{4} \mathrm{~F}_{31}-x\end{array}$ & 4 & $-\ldots--$ & (1. 237) & 1. 20 & $.20 \mathrm{w}$ & 1. $02+$ \\
\hline 60 & 2482.78 & 40265.2 & $b^{4} \mathrm{D}_{21 / 2}-w^{4} \mathrm{D}_{21 / 2}^{0}$ & 6 & $(0.044)$ & 1. 345 & 1. 301 & .110 & 1. 323 \\
\hline 1 & 2483. 20 & 40258.5 & $-\ldots-$ & & $\ldots$ & - - - - & $-\ldots-\ldots$ & - n- & \\
\hline 3 & 2483. 54 & 40253. 0 & 的- & & & $---\bar{x}--$ & ----- & $-\cdots---$ & $---10-1$ \\
\hline $\begin{array}{r}50 \\
5\end{array}$ & $\begin{array}{l}\text { 2483. } 96 \\
2484.49\end{array}$ & $\begin{array}{l}\text { 40246. } 2 \\
40237.5\end{array}$ & 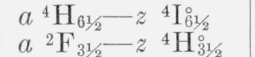 & 6 & $(0.10)$ & 1. 23 & 1. 03 & $.67-$ & 1. $13 \pm$ \\
\hline 10 & 2484. 67 & 40234. 6 & $b^{4} \mathrm{D}_{01 / 2}^{3 / 2}-x{ }^{4} \mathrm{P}_{01 / 2}^{0}$ & 6 & 1. 62 & 0.14 & 1. 76 & .81 & 0.95 \\
\hline 8 & 2487. 20 & 40193. 7 & $b^{4} \mathrm{D}_{21 / 2}-y{ }^{6} \mathrm{P}_{31 / 2}^{\circ}$ & $5^{*}$ & 0.268 & 1. 306 & 1. 574 & .135 & 2. $244 ?$ \\
\hline $\begin{array}{l}4 H \\
3\end{array}$ & $\begin{array}{l}\text { 2487. } 51 \\
2487.90\end{array}$ & $\begin{array}{l}40188.7 \\
40182.4\end{array}$ & $\begin{array}{l}b{ }^{4} \mathrm{P}_{21 / 2}-z{ }^{2} \mathrm{~F}_{21 / 2}^{21 / 2} \\
a^{4} \mathrm{H}_{31 / 2}-x{ }^{4} \mathrm{D}_{21 / 2}^{2}\end{array}$ & $-\cdots$ & - n & $\ldots \ldots$ & - n- & $-\cdots-1$ & $\ldots \ldots-$ \\
\hline 30 & 2488. 57 & 40171. 6 & $b^{4}{ }^{4} \mathrm{~F}_{31 / 2}^{31 / 2}-z{ }^{2} \mathrm{H}_{41 / 2}^{212}$ & 4 & - n & $(1.093)$ & 1. 02 & $.23 w$ & $0.76+$ \\
\hline 3 & 2488.88 & 40166.6 & 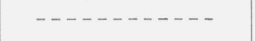 & $-\cdots--$ & $\ldots \ldots$ & 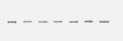 & -.....- & $\ldots \ldots$ & $-\ldots \ldots$ \\
\hline 50 & 2489. 34 & 40159. 2 & $a^{2} \mathrm{I}_{51 / 2}-w^{2} \mathrm{H}_{41 / 2}^{0}$ & 4 & ....... & $(0.90)$ & 0.91 & $w$ & .87 \\
\hline 6 & 2490.54 & 40139.8 & $a^{4} \mathrm{H}_{51 / 2}-z{ }^{2} \mathrm{I}_{51 / 2}$ & $\ldots-$ & $\ldots \ldots$ & - $-\ldots$ & $\ldots \ldots$ & $\ldots-\ldots-$ & $\ldots+\ldots$ \\
\hline 50 & 2491. 08 & 40131. 2 & $a^{4} \mathrm{G}_{31 / 2}-y{ }^{2} \mathrm{D}_{21 / 2}^{2}$ & $\ldots-$ & $\ldots \ldots$ & $\ldots-\ldots$ & $\ldots-\ldots$ & $-\ldots-1$ & $\ldots \ldots$ \\
\hline $\begin{array}{r}40 \\
5\end{array}$ & $\begin{array}{l}\text { 2491. } 10 \\
2491.34\end{array}$ & $\begin{array}{l}40130.8 \\
40127.0\end{array}$ & $\begin{array}{l}a{ }^{4} \mathrm{H}_{41 / 2}-z{ }^{2} \mathrm{I}_{51 / 2}^{0} \\
b^{4} \mathrm{~F}_{21 / 2}-y{ }^{2} \mathrm{G}_{31 / 2}^{0}\end{array}$ & $-\overline{5}^{---}$ & $\ldots$ & $(0.943)$ & 1. 028 & $w^{-\cdots}$ & 1. $24-$ \\
\hline 40 & 2491.56 & 40123,4 & $b^{4} \mathrm{D}_{21}-w^{4} \mathrm{~F}_{316}^{\circ}$ & 7,4 & & (1 344$)$ & 1.32 & 0 & 126 \\
\hline $\begin{array}{r}40 \\
5\end{array}$ & 2493. 59 & 40090.9 & $b^{4} \mathrm{~F}_{21 / 2}-x{ }^{4} \mathrm{D}_{31 / 2}^{11 / 2}$ & 7,4 & - n- & $(1.344)$ & $\begin{array}{l}1.32 \\
-\ldots . .-\end{array}$ & 0 & 1. 26 \\
\hline 300 & 2493. 68 & 40089. 2 & $a^{4} \mathrm{H}_{51 / 2}-z^{4} \mathrm{I}_{61 / 2}^{0}$ & $7 *$ & - & - n & - n & 0 & 1. 124 \\
\hline 10 & 2494. 16 & 40081.6 & $b^{4} \mathrm{D}_{11 / 2}-w^{4} \mathrm{~F}_{21 / 2}^{0}$ & & $---\overline{-n--}$ & & & & \\
\hline 40 & 2494. 48 & 40076. 4 & $a{ }^{6} \mathrm{D}_{11 / 2}-z{ }^{6} \mathrm{~F}_{01 / 2}^{0}$ & 5 & 2. 50 & 1. 83 & -0.67 & 1. 25 & 3. 08 \\
\hline $2 h$ & 2495. 35 & 40062.4 & & & - - - & & & & \\
\hline $\begin{array}{l}200 \\
2 h\end{array}$ & $\begin{array}{l}2495.69 \\
2495.94\end{array}$ & 40057. 0 & $b{ }^{4} \mathrm{~F}_{41 / 2}-y{ }^{4} \mathrm{G}_{51 / 2}^{\circ}$ & 4 & $\ldots \ldots$ & $(1.200)$ & 1. 17 & $w$ & 1. $03+$ \\
\hline 25 & 2496. 86 & $\begin{array}{l}40053.0 \\
40038.2\end{array}$ & $-\ldots-n----$ & 6 & - & 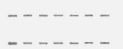 & - & $0.26-$ & $0.98 \pm$ \\
\hline 1 & 2497. 36 & 40030. 2 & $b^{4} \mathrm{D}_{31 / 2}-x{ }^{2} \mathrm{H}_{41 / 2}^{\circ} ?$ & $-\ldots-$ & -....- & $-\ldots \ldots$ & $\ldots \ldots$ & - . - . - & $-\ldots \ldots$ \\
\hline 200 & 2498. 41 & 40013. 4 & $a^{6} \mathrm{D}_{11 / 2}-z{ }^{6} \mathrm{~F}_{11 / 2}^{0}$ & $?$ & & & & & \\
\hline 200 & 2498.58 & 40010.7 & $a^{6} \mathrm{D}_{21 / 2}-z{ }^{6} \mathrm{~F}_{31 / 2}^{0}$ & 4 & 0.180 & 1. 637 & 1. 457 & .091 & 1. 007 \\
\hline $\begin{array}{l}8 \\
3\end{array}$ & $\begin{array}{l}2499.38 \\
2499.56\end{array}$ & $\begin{array}{l}39997.8 \\
309940\end{array}$ & $b{ }^{4} \mathrm{~F}_{31 / 2}-y{ }^{4} \mathrm{G}_{21 / 2}$ & $-\cdots-$ & 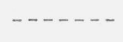 & $\ldots \ldots$ & $\ldots \ldots$ & $\ldots \ldots$ & $-\ldots \ldots$ \\
\hline 50 & 2500. 14 & 39985.6 & $a^{2} \mathrm{I}_{61 / 2}-y^{2} \mathrm{I}_{51 / 2}^{\circ}$ & 5 & $(0.08)$ & 1.08 & 1.00 & $.41 w$ & 1. $52-$ \\
\hline 100 & 2501.95 & 39956.8 & $b{ }^{4} \mathrm{~F}_{41 / 2}-z \quad{ }^{2} \mathrm{H}_{51 / 2}^{0}$ & 4 & $(0.06)$ & 1. 20 & 1. 14 & $.25 w$ & $0.87+$ \\
\hline $40 h$ & 2502.30 & 39951. 3 & $\left\{\begin{array}{l}b{ }^{4} \mathrm{D}_{21 / 2}-w^{4} \mathrm{~F}_{21 / 2}^{0} \\
b^{4} \mathrm{D}_{31 / 2}-w^{4} \mathrm{D}_{21 / 2}^{0}\end{array}\right.$ & $-\cdots$ & $\ldots \ldots$ & - . - & $\ldots \ldots$ & $\ldots \ldots$ & - - n \\
\hline $\begin{array}{l}5 H \\
10\end{array}$ & $\begin{array}{l}2503.22 \\
2504.92\end{array}$ & $\begin{array}{l}39936.5 \\
39909.5\end{array}$ & $b^{2} \mathrm{G}_{41}-y^{2} \mathrm{I}^{0}$ & & & & & $-\ldots-\ldots$ & 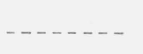 \\
\hline 10 & 2505.64 & & $\left\{a^{2} \mathrm{G}_{41 / 2}-z \quad{ }^{6} \mathrm{~F}_{51 / 2}^{\circ}\right.$ & & & & & & \\
\hline & 2000.04 & 39897.9 & $\left(b{ }^{4} \mathrm{D}_{11 / 2}-2_{11 / 2}^{\circ}\right.$ & & $\cdots \cdots$ & $\ldots \ldots$ & $\cdots \cdots$ & $\ldots-\cdots$ & 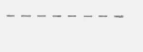 \\
\hline 15 & 2505. 89 & 39894. 0 & $a^{4} \mathrm{H}_{41 / 2}-z^{2} \mathrm{~F}_{31 / 2}^{\circ}$ & $\ldots-$ & - - - - & $\ldots \ldots$ & - - n & - - - - - & $\ldots-\ldots$ \\
\hline $\begin{array}{l}25 \\
25\end{array}$ & 2506. 25 & 39888. 2 & $a^{4} \mathrm{G}_{51 / 2}-x{ }^{4} \mathrm{G}_{41 / 2}^{0}$ & $-\cdots$ & $\ldots \ldots$ & $\ldots \ldots$ & $\ldots \ldots$ & $\ldots \ldots$ & $\ldots \ldots$ \\
\hline $\begin{array}{r}25 \\
300\end{array}$ & $\begin{array}{l}2506.42 \\
2507.00\end{array}$ & $\begin{array}{l}39885.5 \\
39876.3\end{array}$ & $\begin{array}{l}b^{4} \mathrm{D}_{31 / 2}-w^{4} \mathrm{~F}_{41 / 2}^{0} \\
a^{6} \mathrm{D}_{11 / 2}-z{ }^{6} \mathrm{~F}_{21 / 2}^{0}\end{array}$ & 4 & 0.536 & 1. 869 & 1.333 & $26 \overline{6}$ & 0.592 \\
\hline & 2507.50 & 39868. 4 & & & & & & & \\
\hline
\end{tabular}


TABLE 6. The second spectrum of ruthenium (Ru II) - Continued

\begin{tabular}{|c|c|c|c|c|c|c|c|c|c|}
\hline $\begin{array}{c}1 \\
\text { Intensity }\end{array}$ & $\begin{array}{c}2 \\
\lambda(\text { air })\end{array}$ & $\begin{array}{l}3 \\
\sigma\end{array}$ & $\begin{array}{c}4 \\
\text { Term combina- } \\
\text { tion }\end{array}$ & $\begin{array}{c}5 \\
\text { Type }\end{array}$ & $\begin{array}{l}6 \\
\Delta g\end{array}$ & 7 & $\begin{array}{l}8 \\
g\end{array}$ & $\begin{array}{c}9 \\
\text { Strong } p\end{array}$ & $\begin{array}{c}10 \\
\text { Strong } n\end{array}$ \\
\hline $\begin{array}{c}100 \\
3 \\
6 H \\
1 \\
5 H\end{array}$ & $\begin{array}{c}A \\
2508.67 \\
2509.42 \\
2511.22 \\
2511.59 \\
2512.52\end{array}$ & $\begin{array}{c}K \\
39849.8 \\
39837.8 \\
39809.3 \\
39803.4 \\
39788.7\end{array}$ & 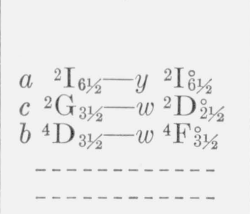 & $\begin{array}{l}7 \\
-\cdots- \\
-\cdots- \\
-\cdots-\end{array}$ & $\begin{array}{l}(0.00) \\
-\cdots-- \\
-\cdots-- \\
---\cdots \\
--\cdots\end{array}$ & \begin{tabular}{c}
1.08 \\
$-\cdots--$ \\
$-\cdots--$ \\
\hdashline$-\cdots$
\end{tabular} & 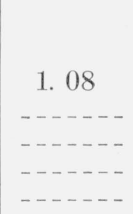 & $\begin{array}{c}0 \\
-\cdots-- \\
-\cdots-- \\
-\cdots--\end{array}$ & \begin{tabular}{l}
1.08 \\
$-1-1-$ \\
\hdashline$-\cdots$
\end{tabular} \\
\hline $\begin{array}{c}150 \\
1 \\
3 h \\
8 \\
10\end{array}$ & $\begin{array}{l}2513.32 \\
2513.61 \\
2514.16 \\
2515.63 \\
2516.71\end{array}$ & $\begin{array}{l}39776.0 \\
39771.5 \\
39762.8 \\
39739.5 \\
39722.4\end{array}$ & 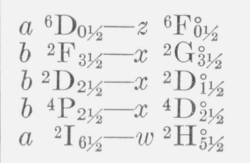 & $\begin{array}{c}6 \\
-6 u s \\
-\cdots--\end{array}$ & $\begin{array}{l}3.87 \\
-\cdots-- \\
-\cdots--\end{array}$ & \begin{tabular}{c}
3.28 \\
$\cdots-\ldots$ \\
\hdashline$-\ldots-$
\end{tabular} & $\begin{array}{l}-0.59 \\
-\cdots \\
\cdots\end{array}$ & \begin{tabular}{c}
1.935 \\
\hdashline$-1-$ \\
$-1-1$
\end{tabular} & $\begin{array}{l}1.347 \\
-\cdots\end{array}$ \\
\hline $\begin{array}{r}200 \\
100 \\
75 \\
10 \\
40\end{array}$ & $\begin{array}{l}2517.32 \\
2518.41 \\
2519.20 \\
2520.57 \\
2520.82\end{array}$ & $\begin{array}{l}39712.8 \\
39695.6 \\
39683.2 \\
39661.6 \\
39657.6\end{array}$ & 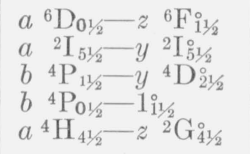 & $\begin{array}{l}4 \\
6 \\
4 \\
-6 u s\end{array}$ & $\begin{array}{l}2.21 \\
(0.05) \\
0.327 \\
\cdots\end{array}$ & $\begin{array}{l}\text { 3. } 26 \\
0.90 \\
\text { 1. } 634 \\
\text { 1. } 14\end{array}$ & $\begin{array}{l}\text { 1. } 05 \\
0.95 \\
\text { 1. } 307 \\
1.00\end{array}$ & $\begin{array}{l}\text { 1. } 11 \\
0.29 \\
.164 \\
-.20\end{array}$ & $\begin{array}{c}-0.06 ? \\
.93 \\
.817 \\
1.07\end{array}$ \\
\hline $\begin{array}{r}3 \\
25 \\
1 \\
8 \\
1\end{array}$ & $\begin{array}{l}\text { 2522. } 26 \\
2522.59 \\
2522.74 \\
2523.16 \\
2523.72\end{array}$ & $\begin{array}{l}39634.9 \\
39629.9 \\
39627.5 \\
39620.9 \\
39612.1\end{array}$ & $b^{{ }^{4} \mathrm{D}_{11 / 2}-w^{4} \mathrm{~F}_{11 / 2}^{\circ}}$ & $\begin{array}{l}-\cdots \\
-\cdots \\
-\cdots-\end{array}$ & $(0.05)$ & $(1.200)$ & 1.15 & 0.23 & 1. 101 \\
\hline $\begin{array}{l}20 \\
100 \\
200 H \\
25 \\
20\end{array}$ & $\begin{array}{l}2524.39 \\
2524.85 \\
2525.42 \\
2526.88 \\
2527.07\end{array}$ & $\begin{array}{l}39601.7 \\
39594.4 \\
39585.5 \\
39562.5 \\
39559.6\end{array}$ & 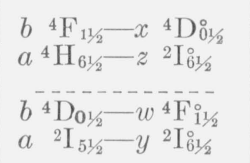 & $\begin{array}{l}7 \\
6 \\
-5 \\
----\end{array}$ & $\begin{array}{l}(0.08) \\
0.368\end{array}$ & $\begin{array}{l}(0.415) \\
1.20 \\
0.131 \\
-\end{array}$ & $\begin{array}{l}0.40 \\
1.12 \\
0.499 \\
-\end{array}$ & $\begin{array}{l}0 \\
0.51 \\
-184 \\
- \\
-\end{array}$ & $\begin{array}{l}0.42 \\
1.16 \pm \\
0.683 \\
-. .--\end{array}$ \\
\hline $\begin{array}{r}3 \\
200 \\
100 \\
20 \\
10\end{array}$ & $\begin{array}{l}2527.30 \\
2527.86 \\
2528.05 \\
2529.61 \\
2530.04\end{array}$ & $\begin{array}{l}39556.0 \\
39547.2 \\
39544.3 \\
39520.0 \\
39513.2\end{array}$ & $\begin{array}{l}a^{4} \mathrm{G}_{41 / 2}-y{ }^{4} \mathrm{H}_{31 / 2}^{\circ} \\
a^{4} \mathrm{G}_{41 / 2}-y{ }^{4} \mathrm{H}_{51 / 2}^{0} \\
c^{2} \mathrm{G}_{41 / 2}-w^{2} \mathrm{~F}_{31 / 2}^{0} \\
a^{4} \mathrm{H}_{61 / 2}-z^{4} \mathrm{I}_{51 / 2}^{0} \\
a^{4} \mathrm{G}_{41 / 2}-y{ }^{4} \mathrm{H}_{41 / 2}^{0}\end{array}$ & $\begin{array}{l}-4 \\
7 \\
5 \\
-\cdots-\end{array}$ & $(0.06)$ & $\begin{array}{l}1.17 \\
(1.23)\end{array}$ & 1.11 & $\begin{array}{l}0.29 w \\
0.09\end{array}$ & $\begin{array}{l}\text { 1. } 84+ \\
\text { ? }\end{array}$ \\
\hline $\begin{array}{r}70 \\
2 \\
10 \\
6 \\
100\end{array}$ & $\begin{array}{l}\text { 2530. } 41 \\
2531.68 \\
2533.03 \\
2533.39 \\
2533.97\end{array}$ & $\begin{array}{l}39507.4 \\
39487.6 \\
39466.6 \\
39461.0 \\
39451.9\end{array}$ & $b^{{ }^{4} \mathrm{~F}_{21 / 2}-y{ }^{4} \mathrm{G}_{31 / 2}^{0}}$ & \begin{tabular}{l}
5 \\
-1 \\
\hdashline 5 \\
5
\end{tabular} & $\begin{array}{l}(0.08) \\
-0.56 \\
\quad .268\end{array}$ & $\begin{array}{l}0.92 \\
(1.188) \\
0.426\end{array}$ & $\begin{array}{l}1.00 \\
\text { 1. } 748 \\
0.694\end{array}$ & $\begin{array}{c}.19 w \\
0.28 \\
.134\end{array}$ & $\begin{array}{l}\text { 1. } 20- \\
\text { 1. } 096\end{array}$ \\
\hline $\begin{array}{r}80 \\
50 \\
100 \\
5 \\
3\end{array}$ & $\begin{array}{l}2534.92 \\
2535.23 \\
2535.60 \\
2536.30 \\
2536.68\end{array}$ & $\begin{array}{l}39437.1 \\
39432.4 \\
39426.6 \\
39415.7 \\
39409.8\end{array}$ & 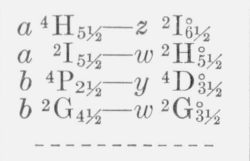 & $\begin{array}{c}? \\
4 \\
-1-\end{array}$ & 0.226 & 1.565 & 1. 339 & 0.111 & 0.774 \\
\hline $\begin{array}{l}10 H w \\
100 \\
100 \\
1 \\
1\end{array}$ & $\begin{array}{l}\text { 2537. } 34 \\
2539.72 \\
2540.30 \\
2540.84 \\
2541.80\end{array}$ & $\begin{array}{l}39399.5 \\
39362.6 \\
39353.6 \\
39345.2 \\
39330.4\end{array}$ & $\begin{array}{c}\left\{\begin{array}{l}a^{4} \mathrm{H}_{51 / 2}-z \\
a^{4} \mathrm{I}_{21 / 2} \mathrm{I}_{51 / 2}^{\circ} y^{4} \mathrm{H}_{31 / 2}^{\circ}\end{array}\right. \\
a^{4} \mathrm{H}_{41 / 2}-z{ }^{4} \mathrm{I}_{51 / 2}^{\circ}\end{array}$ & $\begin{array}{l}-\cdots- \\
-\ldots- \\
-\ldots-\end{array}$ & 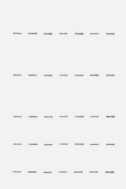 & 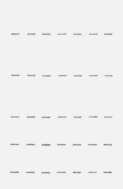 & 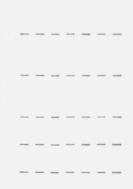 & 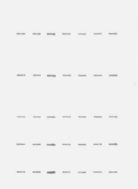 & 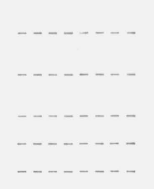 \\
\hline $\begin{array}{r}25 \\
20 \\
100 \\
100 \\
300\end{array}$ & $\begin{array}{l}2542.04 \\
2542.23 \\
2542.146 \\
2543.216 \\
2543.272\end{array}$ & $\begin{array}{l}39326.7 \\
39323.7 \\
39309.6 \\
39308.5 \\
39307.6\end{array}$ & 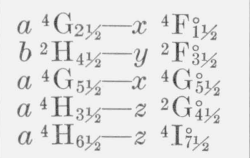 & \begin{tabular}{l}
7 \\
4 \\
\hdashline---
\end{tabular} & \begin{tabular}{c}
$(0.00)$ \\
0.172 \\
\hdashline$(0.03)$
\end{tabular} & $\begin{array}{r}0.648 \\
.958 \\
-1.235\end{array}$ & $\begin{array}{l}0.648 \\
\text { 1. } 130 \\
\text { 1. } 205\end{array}$ & $\begin{array}{l}0 \\
0.086 \\
-.20 \mathrm{w}\end{array}$ & \begin{tabular}{c}
$\begin{array}{c}648 \\
?\end{array}$ \\
\hdashline $1.02+$
\end{tabular} \\
\hline $\begin{array}{c}100 \\
2 \\
1 \\
3 \mathrm{H} w \\
2 H\end{array}$ & $\begin{array}{l}2543.47 \\
2544.18 \\
2545.29 \\
2545.81 \\
2546.40\end{array}$ & $\begin{array}{l}39304.6 \\
39293.6 \\
39276.5 \\
39268.4 \\
39259.3\end{array}$ & $\begin{array}{c}a^{{ }^{4}} \mathrm{G}_{41 / 2}-x{ }^{4} \mathrm{~F}_{31 / 2}^{\circ} \\
b^{2} \mathrm{~F}_{21 / 2}-x{ }^{2} \mathrm{D}_{11 / 2}^{0} \\
a^{4} \mathrm{G}_{21 / 2}-x^{4} \mathrm{G}_{31 / 2}^{0}\end{array}$ & $\begin{array}{l}-\cdots- \\
\cdots-1 \\
\cdots-1 \\
\cdots-1 \\
\cdots-1\end{array}$ & 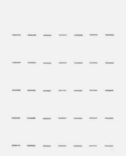 & 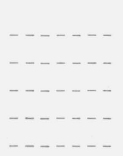 & 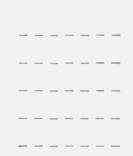 & (n- & 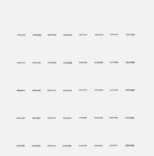 \\
\hline
\end{tabular}


TABLE 6. The second spectrum of ruthenium (Ru II) - Continued

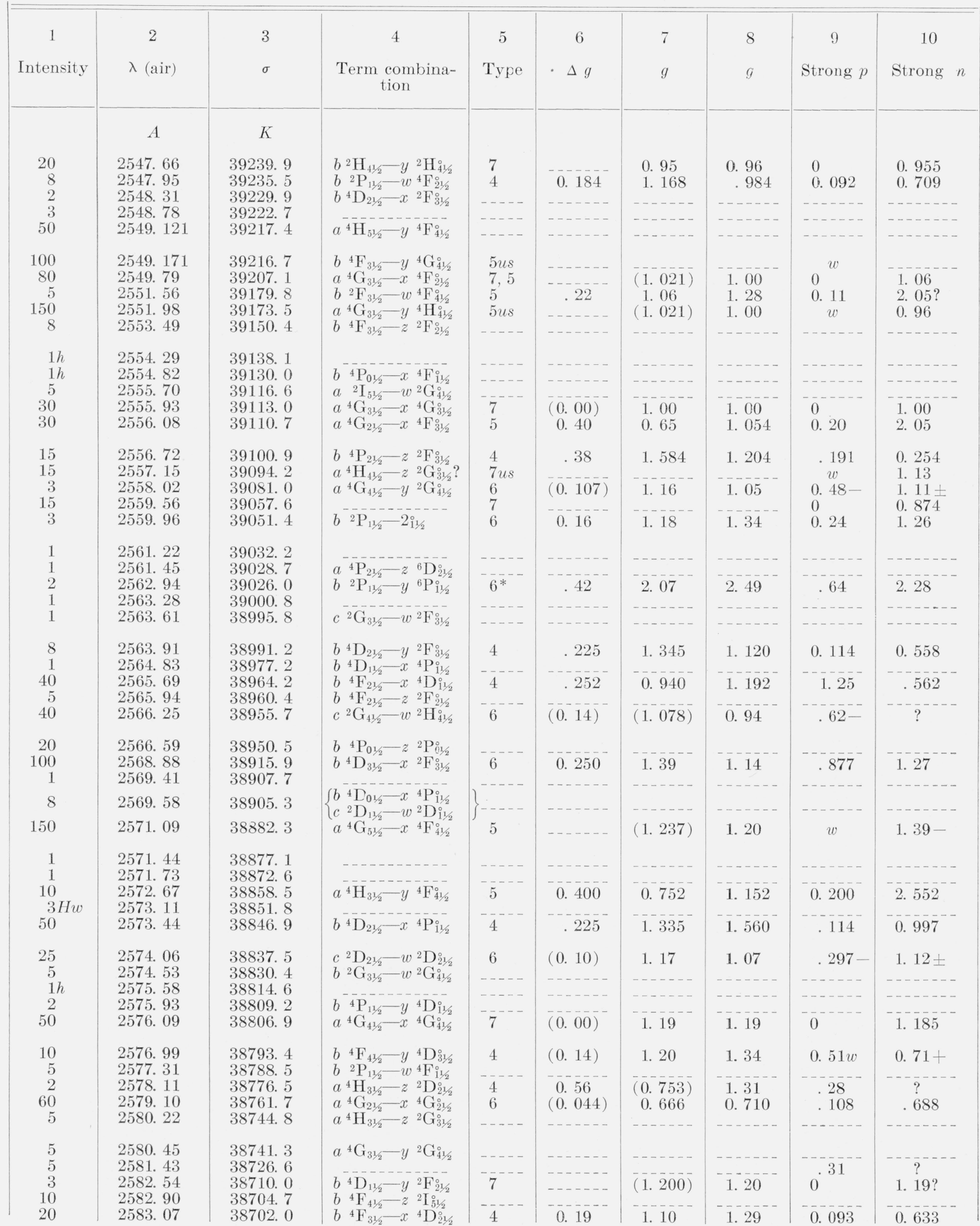


TABLE 6. The second spectrum of ruthenium (Ru II) - Continued

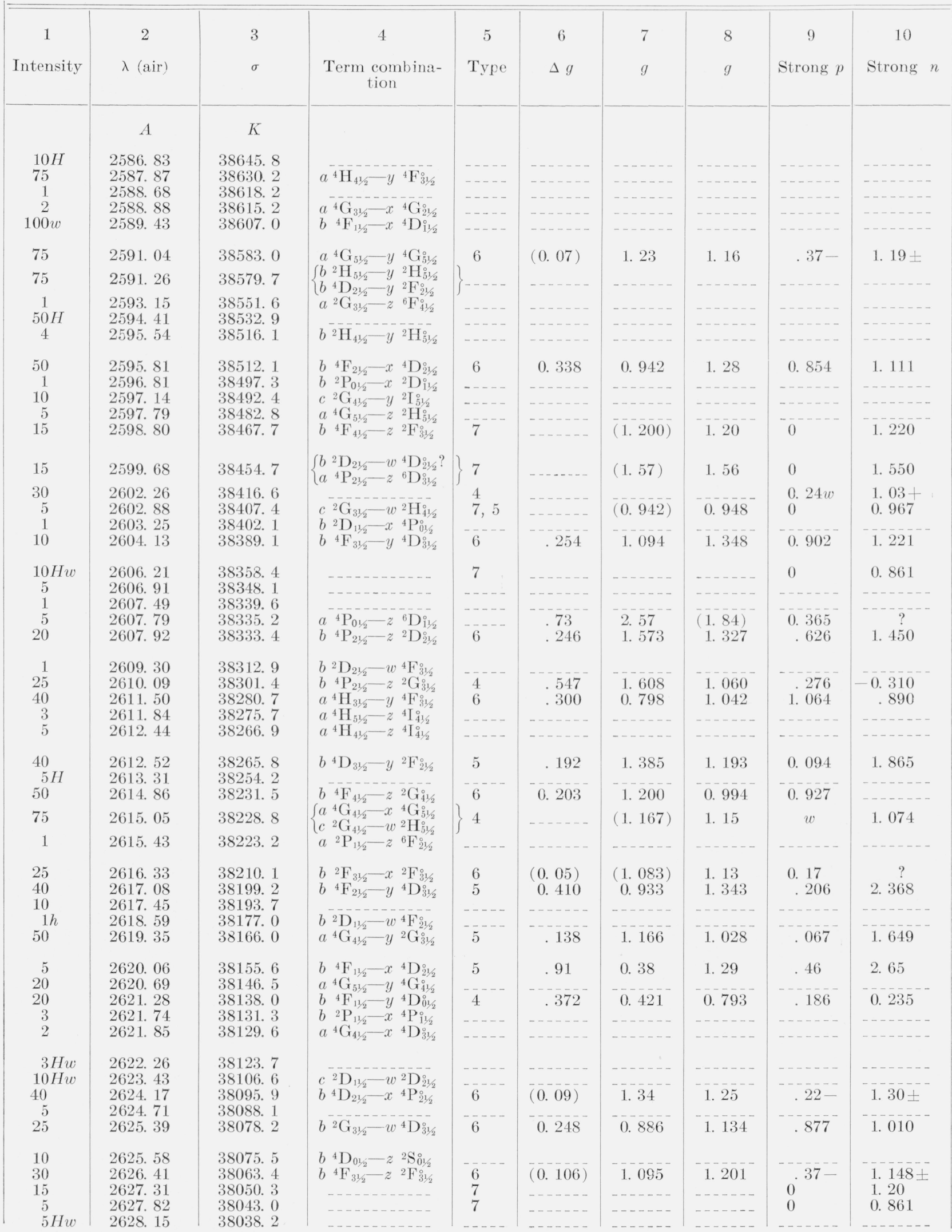


TABLE 6. The second spectrum of ruthenium ( $\mathrm{Ru}$ II) - Continued

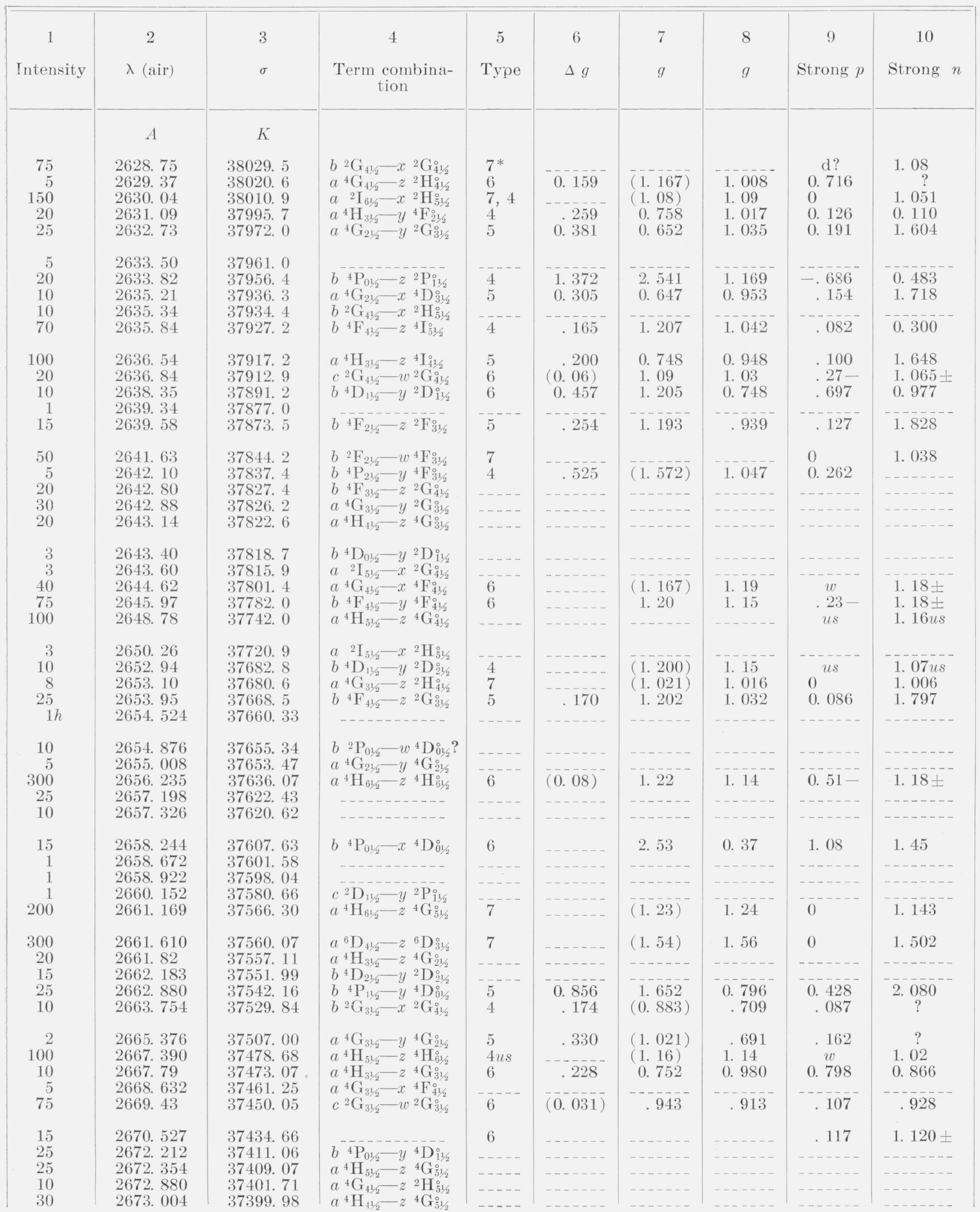


TABLe 6. The second spectrum of ruthenium (Ru II) - Continued

\begin{tabular}{|c|c|c|c|c|c|c|c|c|c|}
\hline $\begin{array}{c}1 \\
\text { Intensity }\end{array}$ & $\begin{array}{c}2 \\
\lambda \text { (air) }\end{array}$ & $\begin{array}{l}3 \\
\sigma\end{array}$ & $\begin{array}{c}4 \\
\substack{\text { Term combina- } \\
\text { tion }}\end{array}$ & $\begin{array}{c}5 \\
\text { Type }\end{array}$ & $\begin{array}{c}6 \\
\Delta g\end{array}$ & $\begin{array}{l}7 \\
g\end{array}$ & $\begin{array}{l}8 \\
g\end{array}$ & $\begin{array}{c}9 \\
\text { Strong } p\end{array}$ & $\begin{array}{c}10 \\
\text { Strong } n\end{array}$ \\
\hline & $A$ & $K$ & & & & & & & \\
\hline 10 & 2674. 219 & 37382.99 & $a^{4} \mathrm{H}_{31 / 2}-z{ }^{4} \mathrm{G}_{41 / 2}^{0}$ & 5 & 0. 380 & $(0.753)$ & 1. 133 & .190 & $?$ \\
\hline 10 & 2675. 197 & $\begin{array}{l}37369.32 \\
37364.49\end{array}$ & $b{ }^{4} \mathbf{F}_{21 / 2}-y{ }^{4} \mathbf{P}_{11 / 2}$ & $-\cdots$ & 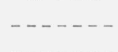 & 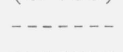 & $-\cdots---$ & 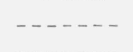 & 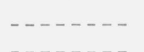 \\
\hline 100 & 2676. 183 & $\begin{array}{l}57004.49 \\
37355.55\end{array}$ & $\left\{\begin{array}{lll}a^{4} \mathrm{H}_{61 / 2} & { }^{4} \mathrm{H}_{51 / 2} \\
a & { }^{6} \mathrm{~S}_{21} & { }^{6} \mathrm{P}^{1}\end{array}\right.$ & 5 & 0.143 & 1. 24 & 1. 10 & $0.79 w$ & $2.03-$ \\
\hline 4 & 2676.775 & 37347. 29 & $b^{2} \mathrm{G}_{41 / 2}-x^{2} \mathrm{H}_{41 / 2}^{\circ}$ & 6 & 104 & (1.067) & 0.96 & 47 & $?$ \\
\hline 3 & 2678. 054 & 37329.46 & $a^{4} \mathrm{H}_{31 / 2}-y{ }^{4} \mathrm{P}_{21 / 2}$ & & & & & & \\
\hline 800 & 2678. 759 & 37319.63 & $a^{6} \mathrm{D}_{41 / 2}-z{ }^{6} \mathrm{D}_{41 / 2}^{0}$ & 7 & $\ldots \ldots$ & 1. 532 & (1. 530$)$ & 0 & 1. 531 \\
\hline $\begin{array}{r}5 \\
100\end{array}$ & $\begin{array}{l}2679.450 \\
2680.585\end{array}$ & $\begin{array}{l}37310.01 \\
37294.21\end{array}$ & $b^{2} \mathrm{G}_{31 / 2}-x^{2} \mathrm{G}_{31 / 2}^{\circ}$ & 6 & .221 & 0.893 & 1. 114 & $\begin{array}{l}0 \\
0.794\end{array}$ & $\begin{array}{l}\text { 1. } 55 \\
1.003\end{array}$ \\
\hline 2 & 2681. 293 & 37284.36 & $a{ }^{6} \mathbf{S}_{21 / 2}^{5 / 2}-w^{4} \mathbf{F}_{31 / 2}^{10}$ & $\ldots$ & $\ldots \ldots$ & $\ldots \ldots$ & 1. 1. 1 & - & - n \\
\hline 1 & 2681. 417 & 37282.64 & & & & & & $\ldots$ & $-\cdots$ \\
\hline 3 & 2682. 632 & 37265. 76 & & & & & & $\ldots \ldots \ldots$ & \\
\hline $\begin{array}{l}5 \\
3\end{array}$ & $\begin{array}{l}\text { 2682. } 754 \\
2682.894\end{array}$ & $\begin{array}{l}37264.06 \\
37262.12\end{array}$ & 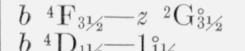 & 6 & $(0.05)$ & 1. 09 & 1. 04 & $.19-$ & 1. $07 \pm$ \\
\hline $1 \tilde{5}$ & 2684.613 & 37238.26 & $b^{4} \mathrm{D}_{31 / 2}-y^{21 / 2} \mathrm{D}_{21 / 2}^{\circ}$ & 5 & 0. 249 & 1. 406 & 1. 157 & .125 & 2. 028 \\
\hline 15 & 2685. 152 & 37230.78 & $a^{4} \mathrm{G}_{51 / 2}-z^{2} \mathrm{I}_{51 / 2}^{\circ}$ & 6 & .197 & (1.237) & 1. 040 & 1. 084 & $?$ \\
\hline 2 & 2685. 581 & 37224.84 & $b{ }^{2} \mathbf{F}_{21 / 2}-w^{4} \mathbf{F}_{11 / 2}^{0}$ & $-\ldots$ & $\ldots \ldots$ & $\ldots \ldots-\ldots$ & $\ldots \ldots \ldots$ & $\ldots \ldots$ & $\ldots \ldots$ \\
\hline $\begin{array}{l}2 \\
3\end{array}$ & $\begin{array}{l}\text { 2685. } 893 \\
2686.418\end{array}$ & $\begin{array}{l}37220.51 \\
37213.24\end{array}$ & $b{ }^{2} \mathrm{P}_{01 / 2}-w{ }^{4} \mathrm{D}_{11 / 2}^{\circ}$ & 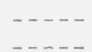 & $\ldots$ & $-\ldots-\ldots$ & $\ldots$ & 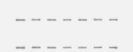 & 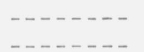 \\
\hline 15 & 2686.889 & 37206.72 & $a^{4} \mathrm{G}_{31 / 2}-y^{4} \mathrm{G}_{31 / 2}^{o}$ & $4 ?$ & - n & $(1.021)$ & 1.03 & $w$ & $1.02+$ \\
\hline 25 & 2687. 071 & 37204.20 & $b{ }^{4} \mathrm{~F}_{41 / 2}-y{ }^{4} \mathrm{~F}_{31 / 2}^{\circ}$ & 5 & .147 & 1. 186 & 1. 039 & 0.072 & 1. $700-$ \\
\hline 100 & 2687. 494 & 37198.34 & $a^{4} \mathrm{H}_{51 / 2}-z{ }^{4} \mathrm{H}_{51 / 2}^{1}$ & $\ldots--$ & $\ldots \ldots$ & $\ldots \ldots \ldots$ & $\ldots \ldots$ & 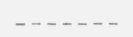 & $\ldots-\ldots$ \\
\hline 80 & $\begin{array}{l}2688.147 \\
2689 \cdot 431\end{array}$ & $\begin{array}{l}37189.31 \\
37171.55\end{array}$ & $a{ }^{4} \mathrm{H}_{41 / 2}-z{ }^{4} \mathrm{H}_{51 / 2}^{0}$ & $-\ldots-$ & $\ldots \ldots$ & $--\ldots-\cdots$ & 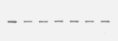 & $-\cdots---$ & $\ldots \ldots-\ldots$ \\
\hline $\begin{array}{l}1 \\
10\end{array}$ & $\begin{array}{l}\text { 2689. } 431 \\
2690.208\end{array}$ & $\begin{array}{l}37171.55 \\
37160.81\end{array}$ & $c^{2} \mathrm{G}_{41 / 2}-w^{4} \mathrm{D}_{31 / 2}^{\circ}$ & 4 & - n & (1.078) & 1. 11 & $w$ & $0.97+$ \\
\hline 400 & 2692. 120 & 37134.43 & $a^{6} \mathrm{D}_{31 / 2}-z{ }^{6} \mathrm{D}_{21 / 2}^{\circ}$ & 4 & $\ldots$ & $(1.576)$ & 1. 71 & $w$ & 1. $44+$ \\
\hline 1 & 2692. 716 & 37126. 21 & $b^{2}{ }^{2} \mathrm{G}_{41 / 2}^{0}-w^{4} \mathrm{~F}_{31 / 2}^{0}$ & $\ldots \ldots$ & $\ldots \ldots$ & $\ldots \ldots$ & $\ldots \ldots$ & $\ldots \ldots$ & $\ldots \ldots$ \\
\hline 20 & 2693. 628 & 37113. 64 & $b \quad{ }^{4} \mathrm{P}_{21 / 2}-z{ }^{4} \mathrm{G}_{21 / 2}$ & $\ldots \ldots$ & $\ldots \ldots$ & $\ldots \ldots$ & $\ldots \ldots$. & $\ldots \ldots$ & $\ldots \ldots$ \\
\hline 1 & 2694. 199 & 37105.77 & $b{ }^{4} \mathrm{~F}_{21 / 2}-z{ }^{2} \mathrm{D}_{21 / 2}^{0}$ & $-\cdots$ & $\ldots \ldots$ & $\ldots \ldots$ & $\ldots \ldots$ & $\ldots \ldots$ & $\ldots \ldots$ \\
\hline 1 & 2694.598 & 37100.28 & $b{ }^{2} \mathrm{P}_{01 / 2}-x{ }^{4} \mathrm{P}_{01 / 2}^{0}$ & & & $\ldots \ldots$ & $-\cdots$ & & $\ldots \ldots$ \\
\hline 2 & 2696. 495 & 37074.18 & $b{ }^{4} \mathrm{~F}_{21 / 2}-z{ }^{2} \mathrm{G}_{31 / 2}^{0}$ & & & & & & \\
\hline 20 & 2697. 07 & 37066. 3 & $a{ }^{2} \mathbf{S}_{01 / 2}-y^{2} \mathrm{P}_{11 / 2}^{\circ}$ & 4 & 0.516 & 1. 284 & 1. 800 & 0.258 & 1. 026 \\
\hline $\begin{array}{l}10 \\
10\end{array}$ & $\begin{array}{l}2697.12 \\
2698.167\end{array}$ & $\begin{array}{l}37065.6 \\
37051.21\end{array}$ & $\begin{array}{l}a^{4}{ }^{4} \mathrm{G}_{41 / 2}-y \\
a{ }^{6}{ }^{4} \mathrm{G}_{21 / 2} \mathrm{G}_{41 / 2}^{\circ 1}-y \\
{ }^{6} \mathrm{P}_{21 / 2}^{0}\end{array}$ & $\ldots$ & $\ldots$ & 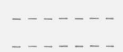 & - & $\ldots \ldots$ & $\ldots$ \\
\hline 20 & 2698. 268 & 37049.82 & - & $-\cdots$ & 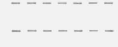 & - n & 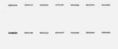 & $w$ & 1. $16 ?$ \\
\hline $3 h$ & 2698. 767 & 37042. 97 & & & & & & & \\
\hline 5 & 2699. 228 & 37036. 64 & $b{ }^{2} \mathrm{D}_{21 / 2}-x{ }^{4} \mathrm{P}_{11 / 2}^{\circ}$ & 4 & . 480 & (1. 102) & 1. 582 & 0. 240 & $?$ \\
\hline 50 & 2700. 163 & 37023. 82 & $b{ }^{2} \mathrm{H}_{51 / 2}-y{ }^{4} \mathrm{H}_{61 / 2}^{0}$ & 6 & $(0.031)$ & 1. 077 & 1. 108 & .172 & 1. 092 \\
\hline 25 & $\begin{array}{l}2700.999 \\
2702\end{array}$ & $\begin{array}{l}37012.36 \\
36989.90\end{array}$ & $b{ }^{4} \mathbf{F}_{11 / 2}-y{ }^{4} \mathrm{P}_{11 / 2}$ ? & 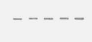 & $\ldots \ldots$ & $\ldots \ldots$ & $\ldots \ldots-\ldots$ & $\ldots \ldots \ldots$ & 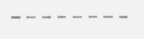 \\
\hline 10 & 2702.639 & 36989.90 & $b^{2} \mathrm{H}_{51 / 2}-y{ }^{4} \mathrm{H}_{41 / 2}^{0}$ & $-\cdots$ & $\cdots \cdots$ & $\ldots \ldots$ & ------ & ----- & 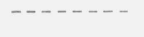 \\
\hline 15 & 2704. 192 & 36968. 66 & $b{ }^{2} \mathrm{H}_{41 / 2}-y{ }^{4} \mathrm{H}_{31 / 2}^{\circ}$ & 5 & 0.146 & 0.958 & 0.812 & .073 & 1. 469 \\
\hline 80 & 2704.585 & 36963. 29 & $a \quad{ }^{2} \mathrm{I}_{61 / 2}-z \quad{ }^{2} \mathrm{~K}_{71 / 2}^{\circ}$ & 4 & $\ldots \ldots$ & $(1.08)$ & 1. 07 & $w$ & $0.99 \div$ \\
\hline 10 & 2704. 830 & 36959. 94 & b ${ }^{2} \mathrm{H}_{41 / 2}-y{ }^{4} \mathrm{H}_{51 / 2}^{0}$ & 5 & .160 & 0.98 & 1. 14 & 0.080 & 1. $70 ?$ \\
\hline 1 & 2705.568 & 36949.86 & $b{ }^{2} \mathrm{~F}_{21 / 2}-x^{2} \mathrm{~F}_{31 / 2}^{0}$ & $\ldots \ldots$ & $\ldots \ldots-\ldots$ & $\ldots \ldots$ & $\ldots \ldots-\ldots$ & $\ldots \ldots$ & $\ldots \ldots$ \\
\hline $1 h$ & 2706. 936 & 36931. 19 & $\ldots-\ldots \ldots$ & $-\ldots$ & $\ldots \ldots$ & $\ldots \ldots$ & $\ldots \ldots$ & $\ldots \ldots$ & $\ldots \ldots$ \\
\hline 50 & 2707. 310 & 36926. 09 & $b^{2} \mathrm{H}_{41 / 2}-y{ }^{4} \mathrm{H}_{41 / 2}^{\circ}$ & 6 & $(0.04)$ & .95 & 0. 99 & $.19-$ & 0.97 \\
\hline 2 & 2707. 555 & 36922. 74 & $-\cdots-1, \ldots$ & $-\ldots$ & $\ldots \ldots$ & $\ldots \ldots$ & $\ldots-\ldots$ & $-\cdots--$ & $\ldots+\cdots$ \\
\hline $\begin{array}{r}1 \\
100\end{array}$ & $\begin{array}{l}2709.000 \\
2710.228\end{array}$ & $\begin{array}{l}36903.05 \\
36886.33\end{array}$ & 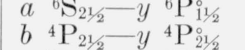 & $-\overline{6}$ & 0. 281 & 1. 576 & 1. 295 & .709 & 1. 436 \\
\hline 300 & 2712. 409 & 36856.67 & $a^{6} \mathrm{D}_{21 / 2}-z{ }^{6} \mathrm{D}_{11 / 2}^{0}$ & 4 & .201 & 1. 637 & 1. 838 & 101 & 1. 335 \\
\hline 40 & 2713. 071 & 36847. 68 & $b^{2} \mathrm{G}_{31 / 2}-x{ }^{2} \mathrm{H}_{41 / 2}^{0}$ & & & & & & \\
\hline 40 & 2713. 585 & 36840. 70 & $b{ }^{4} \mathrm{~F}_{41 / 2}-z{ }^{4} I_{41 / 2}^{\circ}$ & 6 & .247 & 1. 203 & 0.956 & 1. 120 & 1. 080 \\
\hline 1 & 2714. 717 & $\begin{array}{l}36825.34 \\
36820\end{array}$ & $-\cdots-\cdots$ & $-\cdots$ & $\ldots \ldots$ & $\ldots-\cdots$ & $\ldots-\cdots$ & 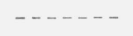 & $\ldots \ldots-\ldots$ \\
\hline $\begin{array}{r}1 \\
30\end{array}$ & $\begin{array}{l}2715.101 \\
2716.132\end{array}$ & $\begin{array}{l}36820.13 \\
36806.16\end{array}$ & $a^{4} \mathrm{G}_{21 / 2}-z^{2} \mathrm{~F}_{21 / 2}^{\circ}$ & 6 & .19 & 0.65 & .84 & 0.46 & 0.745 \\
\hline
\end{tabular}


TABLE 6. The second spectrum of ruthenium (Ru II) - Continued

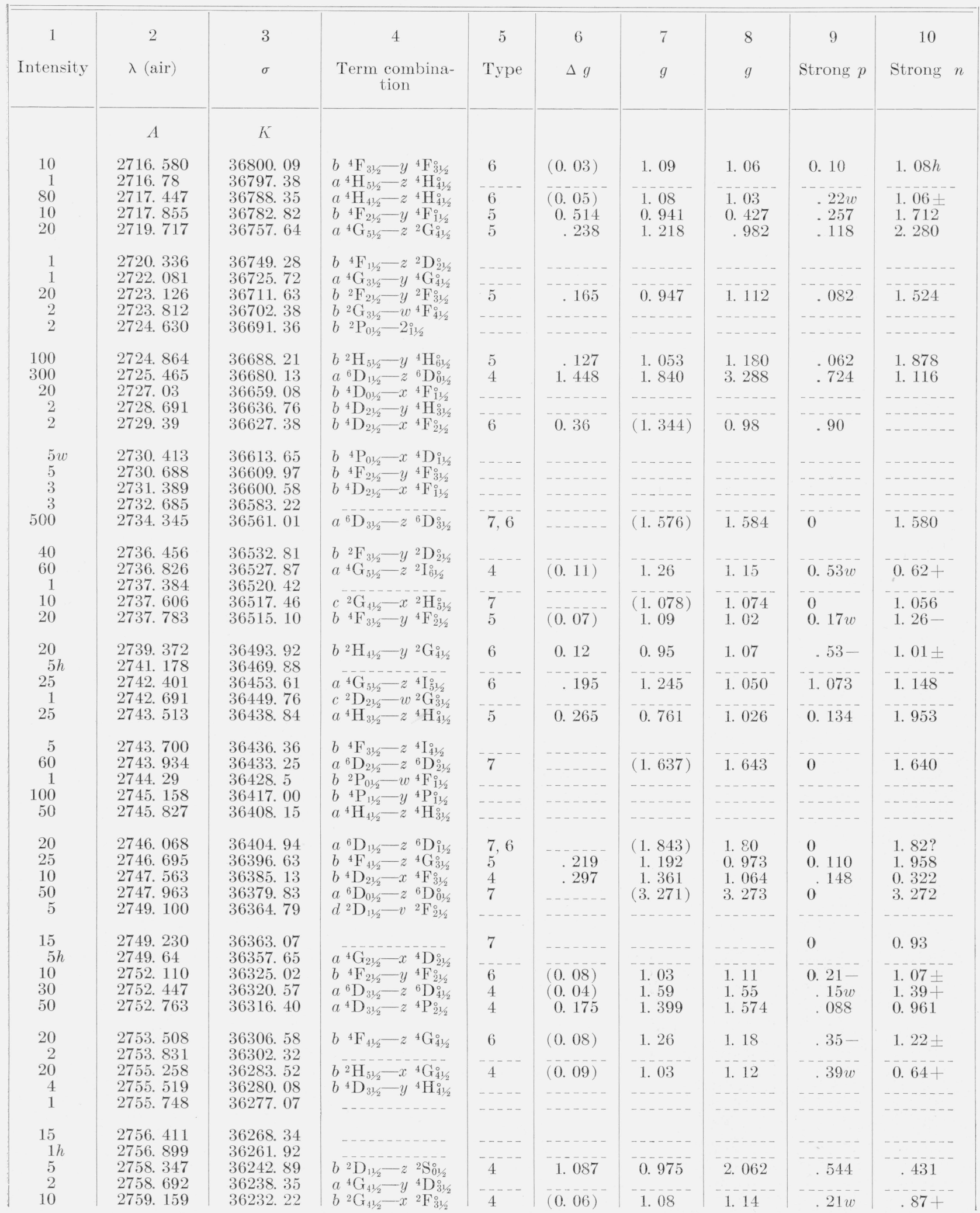


TABLE 6. The second spectrum of ruthenium (Ru II) - Continued

\begin{tabular}{|c|c|c|c|c|c|c|c|c|c|}
\hline $\begin{array}{c}1 \\
\text { Intensity }\end{array}$ & $\begin{array}{c}2 \\
\lambda(\text { air })\end{array}$ & $\begin{array}{l}3 \\
\sigma\end{array}$ & $\begin{array}{c}4 \\
\text { Term combina- } \\
\text { tion }\end{array}$ & $\begin{array}{c}5 \\
\text { Type }\end{array}$ & $\begin{array}{c}6 \\
\Delta g\end{array}$ & $\begin{array}{l}7 \\
g\end{array}$ & $\begin{array}{l}8 \\
g\end{array}$ & $\begin{array}{c}9 \\
\text { Strong } p\end{array}$ & $\begin{array}{c}10 \\
\text { Strong } n\end{array}$ \\
\hline & $A$ & $K$ & & & & & & & \\
\hline 8 & 2760. 115 & 36219. 67 & $b{ }^{2} \mathrm{H}_{41 / 2}-x{ }^{4} \mathrm{G}_{41 / 2}^{\circ}$ & & & & & & \\
\hline $\begin{array}{r}20 \\
3\end{array}$ & $\begin{array}{l}2760.745 \\
2761.473\end{array}$ & $\begin{array}{l}36211.41 \\
36201.86\end{array}$ & $a^{4} \mathrm{G}_{31 / 2}-x{ }^{4} \mathrm{D}_{21 / 2}^{0}$ & 4 & 0.260 & 1. 021 & 1. 281 & .130 & .371 \\
\hline 40 & 2762.071 & 36194. 03 & $d^{2} \mathrm{D}_{21 / 2}-v{ }^{2} \mathrm{~F}_{31 / 2}^{0}$ & 4 & 0.160 & 1. 206 & 1. 046 & 0.080 & 0. 646 \\
\hline 20 & 2765.134 & 36153.93 & $b{ }^{4} \mathrm{P}_{11 / 2}-z{ }^{2} \mathrm{D}_{21 / 2}^{0}$ & 4 & .322 & 1. 643 & 1. 321 & .160 & .838 \\
\hline 100 & 2765.429 & 36150.08 & $a^{4} \mathrm{D}_{21 / 2}-z{ }^{4} \mathrm{P}_{11 / 2}^{\circ}$ & 4 & .372 & 1. 339 & 1. 711 & .186 & .781 \\
\hline 15 & 2765. 889 & 36144. 06 & $b{ }^{4} \mathrm{P}_{01 / 2}-y{ }^{4} \mathrm{D}_{01 / 2}^{\circ}$ & & & & & & \\
\hline $\begin{array}{r}80 \\
250\end{array}$ & 2766.563 & 36135. 26 & $c^{2} \mathrm{D}_{21 / 2}-x{ }^{2} \mathrm{D}_{21 / 2}^{2}$ & 7 & $\ldots \ldots$ & (1. 18) & 1. 20 & 0 & 1. 19 \\
\hline 250 & $\begin{array}{l}2768.926 \\
2770.088\end{array}$ & $\begin{array}{l}36104.42 \\
36089.28\end{array}$ & $a^{6} \mathrm{D}_{01 / 2}-z{ }^{6} \mathrm{D}_{11 / 2}$ & 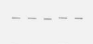 & $\ldots \ldots$ & $\ldots \ldots$ & $\ldots \ldots$ & $\ldots \ldots \ldots$ & $\ldots \ldots-$ \\
\hline & & & & & & & & & \\
\hline$\cdot 50$ & $\begin{array}{l}2771.060 \\
2771.464\end{array}$ & $\begin{array}{l}36076.62 \\
36071.36\end{array}$ & 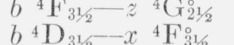 & 5 & .446 & 1. 085 & 0.639 & .223 & 2. 200 \\
\hline 3 & 2772.011 & 36064. 24 & $c^{2} \mathrm{G}_{31 / 2}-x{ }^{2} \mathrm{G}_{41 / 2}^{01 / 2}$ & & $-\ldots-1$ & -... & 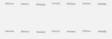 & - n & \\
\hline 100 & 2772. 459 & 36058.42 & $a{ }^{4} \mathrm{H}_{31 / 2}-z{ }^{4} \mathrm{H}_{31 / 2}$ & 6 & .200 & 0.764 & .964 & .708 & 0.864 \\
\hline 10 & 2774.197 & 36035.83 & $b{ }^{4} \mathrm{D}_{21 / 2}-x{ }^{4} \mathrm{G}_{21 / 2}^{0}$ & -- & $\ldots \ldots--$ & $\ldots \ldots \ldots$ & 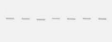 & $-\ldots \ldots$ & $-\ldots \ldots-\ldots$ \\
\hline 30 & 2775. 631 & 36017.21 & $a^{4} \mathrm{D}_{11 / 2} z{ }^{4} \mathrm{P}_{01 / 2}^{0}$ & 4 & 1. 452 & 1. 189 & 2. 641 & .728 & .463 \\
\hline 50 & 2777. 401 & 35994. 26 & $b^{2} \mathrm{G}_{41 / 2}-y{ }^{2} \mathrm{~F}_{31 / 2}^{o}$ & 4 & -1 & (1.067) & 1. 10 & w & $.95+$ \\
\hline $\begin{array}{r}50 \\
1\end{array}$ & $\begin{array}{l}2777.54 \\
2778.024\end{array}$ & $\begin{array}{l}35992.46 \\
35986.19\end{array}$ & $\begin{array}{l}b{ }^{4} \mathrm{~F}_{31 / 2}-z{ }^{4} \mathrm{G}_{31 / 2}{ }^{11} \\
b^{2} \mathrm{D}_{11 / 2}-y \\
{ }^{2} \mathrm{D}_{11 / 2}^{1}\end{array}$ & 6 & 0.11 & 1. 10 & 0.99 & $0.38-$ & $1.05 \pm$ \\
\hline 150 & 2778.388 & 35981.48 & $a{ }^{6} \mathrm{D}_{11 / 2}-z{ }^{6} \mathrm{D}_{21 / 2}^{11 / 2}$ & 4 & .210 & 1. 843 & 1. 633 & .105 & 1. 317 \\
\hline 30 & 2778. 975 & 35973.87 & b ${ }^{4} \mathrm{~F}_{41 / 2}-z{ }^{4} \mathrm{G}_{51 / 2}^{0}$ & 5 & $(0.03)$ & 1. 23 & 1. 26 & $.14 w$ & 1. $40-$ \\
\hline 15 & 2779. 406 & 35968.30 & $b^{4} \mathrm{~F}_{11 / 2}-y{ }^{4} \mathrm{~F}_{21 / 2}$ & 5 & 0.614 & 0.415 & 1. 029 & .308 & 1. $948 ?$ \\
\hline $8 h$ & 2780.817 & 35950.05 & $b^{2} \mathrm{D}_{21 / 2}-y^{2} \mathrm{D}_{11 / 2}^{\circ}$ & 5 & .354 & 1. 107 & .753 & .177 & 1. 638 \\
\hline $\begin{array}{r}8 \\
10\end{array}$ & $\begin{array}{l}2782.23 \\
2782.361\end{array}$ & $\begin{array}{l}35931.79 \\
35930.10\end{array}$ & $c^{2} \mathrm{G}_{41 / 2}-x^{2} \mathrm{H}_{41 / 2}$ & $\begin{array}{l}5 \\
6\end{array}$ & $\begin{array}{l}.228 \\
.12\end{array}$ & $\begin{array}{l}\text { 1. } 053 \\
1.08\end{array}$ & $\begin{array}{l}.825 \\
.96\end{array}$ & $\begin{array}{l}.114 \\
.53-\end{array}$ & $\begin{array}{l}\text { 1. } 623 \\
\text { 1. } 02 \pm\end{array}$ \\
\hline 8 & 2783. 69 & 35912.95 & $a^{4} \mathrm{G}_{41 / 2}-z^{2} \mathrm{~F}_{31 / 2}^{0}$ & 7 & & $(1.167)$ & 1. 18 & 0 & 1. 117 \\
\hline 4 & 2783. 799 & 35911.54 & $b{ }^{2} \mathrm{P}_{11 / 2}-x{ }^{4} \mathrm{~F}_{21 / 2}^{\circ}$ & & & & & & \\
\hline 40 & 2784.516 & 35902. 29 & $b{ }^{4} \mathrm{~F}_{31 / 2}-z{ }^{4} \mathrm{G}_{41 / 2}^{0}$ & 5 & $\ldots \ldots$ & (1.093) & 1. 14 & $w$ & 1. $23-$ \\
\hline 10 & 2785. 186 & 35893.66 & $d^{2} \mathrm{D}_{21 / 2}-3_{31 / 2}^{\circ}$ 。 & 4 & .194 & 1. 227 & 1. 033 & 0.097 & 0.548 \\
\hline 75 & 2785.741 & 35886.51 & $b{ }^{4} \mathrm{~F}_{21 / 2}-z^{4} \mathrm{G}_{21 / 2}^{0}$ & 6 & .292 & 0.944 & 0.652 & .743 & .798 \\
\hline $\begin{array}{l}25 \\
15\end{array}$ & $\begin{array}{l}2785.87 \\
2786.383\end{array}$ & $\begin{array}{l}35884.84 \\
35878.24\end{array}$ & $b{ }^{2} \mathrm{P}_{11 / 2}-x{ }^{4} \mathrm{~F}_{11 / 2}$ & $-\cdots$ & & $\ldots \ldots \ldots$ & & $\ldots \ldots$ & \\
\hline 20 & 2787.25 & 35867.08 & $d^{2} \mathrm{D}_{11 / 2}-w^{2} \mathrm{D}_{11 / 2}^{\circ}$ & 6 & $(0.07)$ & 0.80 & 0.87 & $0.10-$ & $0.83 \pm$ \\
\hline 200 & 2787. 823 & 35859. 71 & $a^{6} \mathrm{D}_{21 / 2}-z{ }^{6} \mathrm{D}_{31 / 2}^{0}$ & 4 & $(0.08)$ & 1. 68 & 1. 60 & $.21 w$ & 1. $39+$ \\
\hline 25 & 2788. 729 & 35848.06 & $b^{4} \mathrm{D}_{31 / 2}-y{ }^{2} \mathrm{G}_{41 / 2}$ & 4 & 0.333 & $(1.397)$ & 1. 063 & .167 & \\
\hline 3 & 2789.453 & 35838. 75 & & & & & & & \\
\hline 5 & 2790.082 & 35830.67 & b ${ }^{4} \mathrm{P}_{11 / 2}-y{ }^{4} \mathrm{~F}_{11 / 2}$ & -- & $\ldots \ldots$ & $\ldots \ldots \ldots$ & $\ldots \ldots$ & $\ldots \ldots \ldots$ & $\ldots \ldots \ldots$ \\
\hline $\begin{array}{r}15 \\
5\end{array}$ & 2790. 240 & $\begin{array}{l}35828.65 \\
35816.41\end{array}$ & $c^{2} \mathrm{G}_{31 / 2}-x{ }^{2} \mathrm{G}_{31 / 2}^{0}$ & --- & $\ldots \ldots$ & $\ldots \ldots \ldots$ & $\ldots \ldots$ & $\ldots \ldots$ & $\ldots \ldots \ldots$ \\
\hline 100 & $\begin{array}{l}2792.32 \\
2790\end{array}$ & $\begin{array}{l}35810.41 \\
35801.96\end{array}$ & $b \quad{ }^{4} \mathrm{~F}_{21 / 2}{ }^{21 / 2}-z \quad-z{ }^{4} \mathrm{G}_{31 / 2}^{21 / 2}$ & $-5^{-}$ & & $(0.943)$ & 0. 99 & $w$ & 1. $09-$ \\
\hline 5 & 2794. 092 & 35779. 25 & $b^{2} \mathrm{D}_{11 / 2}-y^{2} \mathrm{D}_{21 / 2}^{\circ}$ ? & & & & & & \\
\hline $20 h$ & 2794. 278 & 35776.87 & $\ldots$ & 5 & .182 & 1. 120 & .938 & 0.092 & 1. 393 \\
\hline 10 & 2795. 349 & 35763.17 & $b{ }^{4} \mathrm{~F}_{41 / 2}-z{ }^{4} \mathrm{H}_{51 / 2}^{\circ}$ & 4 & .134 & $(1.200)$ & 1. 064 & .068 & \\
\hline 8 & 2795.555 & 35760.53 & $4 D^{-}-$ & $-\ldots$ & $\ldots \ldots \ldots$ & $\ldots \ldots$ & $\ldots \ldots \ldots$ & $\ldots \ldots$ & $\ldots \ldots \ldots$ \\
\hline 5 & 2795. 993 & 35754.93 & $b{ }^{4} \mathrm{D}_{21 / 2}-y{ }^{4} \mathrm{D}_{21 / 2}^{\circ}$ & -- & & & & & $\cdots$ \\
\hline 8 & 2796. 461 & 35748. 94 & & $-\ldots$ & $\ldots \ldots-$ & $\ldots \ldots$ & $\ldots-\ldots$ & $\ldots \ldots$ & $\ldots \ldots-\ldots$ \\
\hline 10 & $\begin{array}{l}2797.078 \\
2797.747\end{array}$ & $\begin{array}{l}35741.07 \\
35732.51\end{array}$ & $\begin{array}{l}a{ }^{6} \mathbf{S}_{21 / 2}-y^{2} \mathrm{~F}^{1} \mathrm{~F}_{21 / 2}^{11} \\
b^{2} \mathrm{G}_{31 / 2}-x^{2} \mathbf{F}_{31 / 6}^{0}\end{array}$ & 6 & .254 & $(0.883)$ & 1. 137 & 889 & $?$ \\
\hline 25 & 2798. 779 & 35719.34 & $a^{4} \mathrm{G}_{21 / 2}-z{ }^{2} \mathrm{~F}_{31 / 2}^{502}$ & 5 & .545 & 0.649 & 1. 194 & .274 & 2. 557 \\
\hline 8 & 2799.587 & 35709.03 & $c^{2} \mathrm{G}_{41 / 2}-w^{4} \mathrm{~F}_{31 / 2}$ & $-\ldots$ & $\ldots \ldots \ldots$ & $\ldots \ldots$ & $\ldots \ldots \ldots$ & $\ldots \ldots$ & $\ldots \ldots$ \\
\hline 15 & 2799. 927 & 35704. 70 & $b{ }^{2} \mathrm{P}_{11 / 2}-z^{2} \mathrm{P}_{01 / 2}^{0}$ & 5 & .363 & 1. 197 & 0.829 & .186 & 1. 381 \\
\hline $\begin{array}{l}1 \\
2\end{array}$ & $\begin{array}{l}2800.408 \\
2800.586\end{array}$ & $\begin{array}{l}35698.56 \\
35696.29\end{array}$ & $a^{2} \mathrm{I}_{51 / 3}-y^{2} \mathrm{H}_{41 / 6}^{\circ}$ & & & $(0.90)$ & 91 & $w$ & $0.86 h$ \\
\hline 30 & 2802. 152 & 35676.34 & $a^{4} \mathrm{G}_{41 / 2}-z^{2} \mathrm{G}_{41 / 2}^{0}$ & 6 & .173 & 1. 168 & .995 & 0.776 & 1. 082 \\
\hline 3 & 2802.717 & 35669.15 & $\ldots \ldots \ldots$ & & & - n..... & & & $\ldots \ldots-\ldots$ \\
\hline
\end{tabular}


TABLE 6. The second spectrum of ruthenium ( $\mathrm{Ru}$ II) - Continued

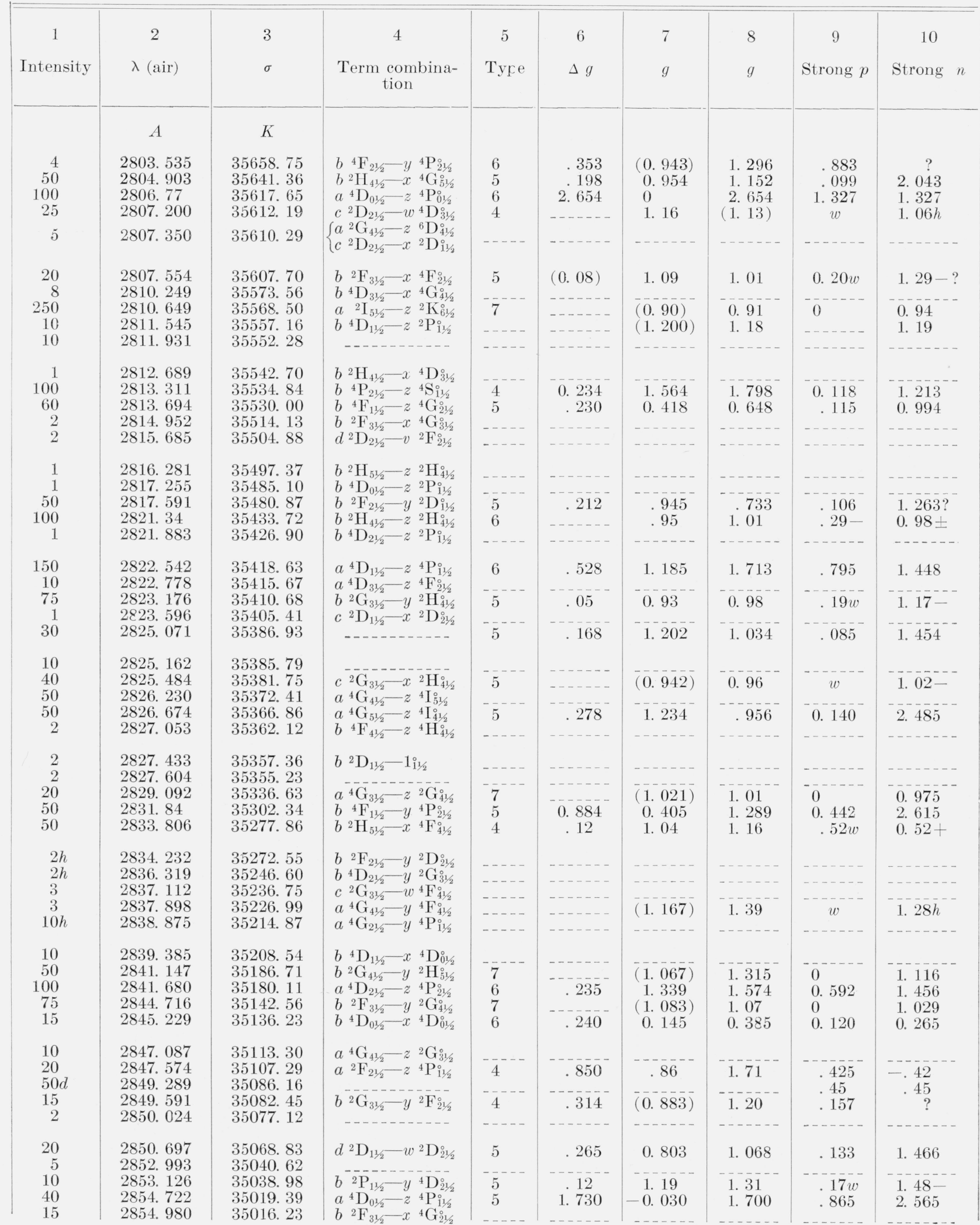


TABLE 6. The second spectrum of ruthenium (Ru II)-Continued

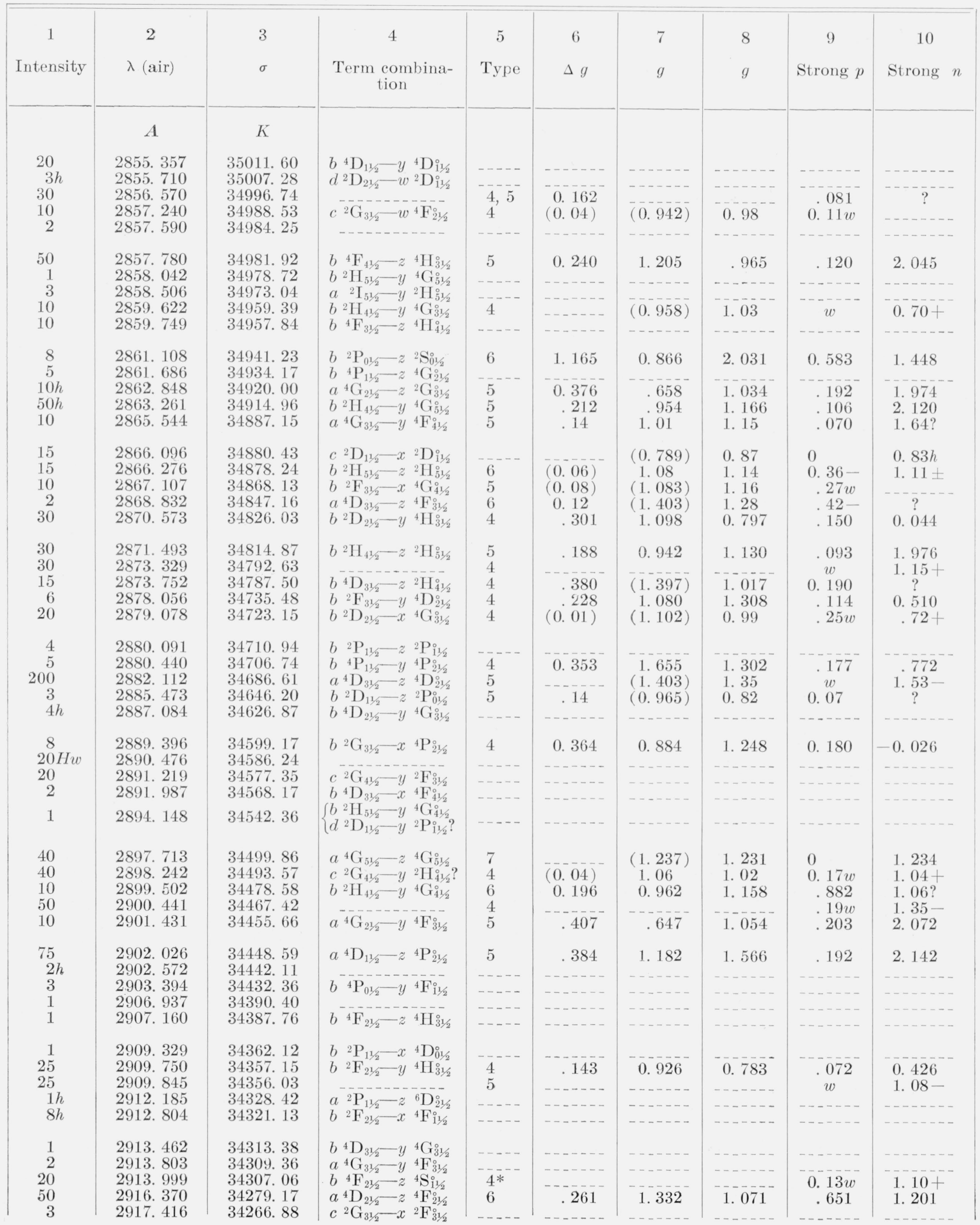


TABLE 6. The second spectrum of ruthenium (Ru II) - Continued

\begin{tabular}{|c|c|c|c|c|c|c|c|c|c|}
\hline $\begin{array}{c}1 \\
\text { Intensity }\end{array}$ & $\begin{array}{c}2 \\
\lambda(\text { air })\end{array}$ & $\begin{array}{l}3 \\
\sigma\end{array}$ & $\begin{array}{c}4 \\
\text { Term combina- } \\
\text { tion }\end{array}$ & $\begin{array}{c}5 \\
\text { Type }\end{array}$ & $\begin{array}{c}6 \\
\Delta g\end{array}$ & $\begin{array}{l}7 \\
g\end{array}$ & $\begin{array}{l}8 \\
g\end{array}$ & $\begin{array}{c}9 \\
\text { Strong } p\end{array}$ & $\begin{array}{c}10 \\
\text { Strong } n\end{array}$ \\
\hline & $A$ & $K$ & & & & & & & \\
\hline $\begin{array}{c}50 \\
1 h\end{array}$ & $\begin{array}{l}2918.521 \\
2920.514\end{array}$ & $\begin{array}{l}34253.90 \\
34230.53\end{array}$ & $b{ }^{2} \mathrm{~F}_{21 / 2}-x{ }^{4} \mathrm{G}_{31 / 2}^{\circ}$ & 5 & $(0.04)$ & 0.94 & 0. 98 & $.11 w$ & 1. $09-$ \\
\hline 10 & 2920. 949 & 34225.43 & $b^{2} \mathrm{D}_{21 / 2}-x^{4} \mathrm{G}_{21 / 2}^{\circ}$ & 6 & 0.402 & 1. 10 & 0.70 & 1. 006 & 0.90 \\
\hline $\begin{array}{l}3 \\
1 h\end{array}$ & $\begin{array}{l}2921.880 \\
2922.192\end{array}$ & $\begin{array}{l}34214.53 \\
34210.87\end{array}$ & $\begin{array}{l}b^{4} \mathrm{D}_{11 / 2}-x{ }^{4} \mathrm{D}_{11 / 2}{ }^{11 / 2} \\
b^{4} \mathrm{D}_{11 / 2}-z{ }^{2} \mathrm{~F}_{21 / 2}^{1}\end{array}$ & $-\ldots-$ & $\ldots \ldots$ & $\ldots \ldots$ & $\ldots \ldots$ & $\ldots \ldots$ & $\ldots \ldots$ \\
\hline 25 & 2922. 347 & 34209.06 & $d^{2} \mathrm{D}_{21 / 2}-w^{2} \mathrm{D}_{21 / 1}^{\circ}$ & 6 & .131 & $(1.20)$ & 1. 07 & 0.328 & $?$ \\
\hline 10 & 2923. 112 & 34200.11 & & 7 & & & & & 0.871 \\
\hline $\begin{array}{l}40 \\
3 H\end{array}$ & $\begin{array}{l}2923.906 \\
2926.519\end{array}$ & $\begin{array}{l}34190.82 \\
34160.29\end{array}$ & $b^{2}{ }^{2} \mathrm{~F}_{31 / 2}-x{ }^{4} \mathrm{D}_{31 / 2}^{\circ}$ & 6 & .122 & 1. 086 & 0. 964 & 0.429 & 1. 025 \\
\hline 200 & $\begin{array}{l}2920.019 \\
2927.535\end{array}$ & $\begin{array}{l}34100.29 \\
34148.44\end{array}$ & $a^{4} \mathrm{D}_{21 / 2}-z^{4} \mathrm{D}_{11 / 2}^{\circ}$ & $-\overline{5}$ & .193 & 1. 337 & 1. 144 & .096 & 1. 626 \\
\hline 2 & 2928. 063 & 34142.28 & $b{ }^{4} \mathrm{D}_{01 / 2}-x{ }^{4} \mathrm{D}_{11 / 2}^{\circ}$ & & & & & & \\
\hline $\begin{array}{r}2 \\
20\end{array}$ & & 34137.34 & $a^{2} \mathrm{~F}_{21 / 2}-z^{4} \mathrm{P}_{21 / 2}$ & & & & & & \\
\hline $\begin{array}{r}20 \\
3\end{array}$ & $\begin{array}{l}\text { 2931. } 209 \\
\text { 2933. } 059\end{array}$ & $\begin{array}{l}34105.64 \\
34084.13\end{array}$ & $\begin{array}{l}b{ }^{2} \mathbf{F}_{21 / 2}-x{ }^{4} \mathbf{F}_{31 / 2}^{0} \\
b^{4} \mathrm{D}_{21 / 2}-x{ }^{4} \mathrm{D}_{11 / 2}^{11}\end{array}$ & $\begin{array}{l}5 \\
5\end{array}$ & $\begin{array}{r}(0.09) \\
0.151\end{array}$ & $\begin{array}{l}0.97 \\
1.333\end{array}$ & $\begin{array}{l}\text { 1. } 06 \\
\text { 1. } 182\end{array}$ & $\begin{array}{l}.23 w \\
.075\end{array}$ & $\begin{array}{l}\text { 1. } 29- \\
\text { 1. } 56 ?\end{array}$ \\
\hline 50 & 2933. 232 & 34082. 12 & $b^{2}{ }^{2} \mathrm{~F}_{31 / 2}^{21 / 2}-z{ }^{2} \mathrm{H}_{41 / 2}^{101 / 2}$ & 4 & $(0.066)$ & (1. 083$)$ & 1. 02 & $23 w$ & $0.78+$ \\
\hline 1 & 2933. 710 & 34076.57 & & & & & & & \\
\hline 40 & 2935.517 & 34055.59 & $b^{2} \mathrm{P}_{01 / 2}-1_{11 / 2}^{i 1}$ & 5 & 0.333 & 0.876 & 1. 209 & .166 & 1. 375 \\
\hline 25 & 2937. 043 & 34037.89 & $c^{2} \mathrm{D}_{11 / 2}-w^{4} \mathrm{D}_{01 / 2}^{\circ}$ & 5 & .242 & .794 & 0. 552 & .121 & 0.915 \\
\hline $1 h$ & 2938. 967 & 34015.61 & D. & $\ldots$ & $\ldots \ldots$ & $\ldots \ldots$ & $\ldots \ldots$ & $\ldots \ldots$ & $\ldots-\ldots$ \\
\hline & 2941. 979 & 33980.79 & $b{ }^{2} \mathrm{D}_{11 / 2}-y{ }^{4} \mathrm{D}_{21 / 2}^{1}$ & -...- & $\ldots \ldots$ & $\ldots \ldots \ldots$ & $\ldots \ldots \ldots$ & $\ldots \ldots$ & $\ldots \ldots \ldots$ \\
\hline 50 & 2942. 244 & 33977.73 & $a^{4} \mathrm{D}_{11 / 2}-z^{4} \mathrm{~F}_{11 / 2}^{0}$ & 6 & .724 & 1. 192 & 468 & 1. 089 & .830 \\
\hline 10 & 2945.098 & 33944. 81 & $c^{2} \mathrm{G}_{31 / 2}^{1 / 2}-y^{2} \mathrm{H}_{41 / 2}^{\circ}$ & 7 & $\ldots-\ldots$ & $(0.942)$ & .95 & 0 & .970 \\
\hline 500 & 2945. 661 & 33938. 32 & $a^{4} \mathrm{D}_{31 / 2}-\approx{ }^{4} \mathrm{D}_{31 / 2}^{\circ}$ & 7 & $\ldots \ldots$ & (1. 403) & 1. 397 & 0 & 1. 400 \\
\hline $1 h$ & 2948. 279 & 33908. 18 & $b^{2} \mathrm{~F}_{31 / 2}-y^{4} \mathrm{G}_{21 / 2}$ & $\ldots-\ldots$ & $\ldots \ldots$ & $\ldots \ldots$ & $\ldots \ldots$ & $\ldots \ldots$ & $--\ldots-\ldots$ \\
\hline 1 & 2948.847 & 33901.65 & $\ldots \ldots-\ldots$ & $\ldots \ldots$ & $\ldots \ldots$ & $\ldots \ldots$ & $\ldots \ldots$ & $\ldots \ldots$ & $\ldots \ldots$ \\
\hline 20 & 2950.028 & 33888.08 & $a^{4} \mathrm{G}_{51 / 2}-z^{4} \mathrm{H}_{41 / 2}^{\circ}$ & 5 & .211 & 1. 231 & 1. 020 & 0.106 & 2. 181 \\
\hline 10 & 2952. 246 & 33862. 62 & $b^{2} \mathbf{F}_{31 / 2}-x^{4} \mathbf{F}_{41 / 2}$ & 5 & .13 & 1. 08 & 1. 21 & $.47 w$ & 1. $54-$ \\
\hline $\begin{array}{l}10 \\
25\end{array}$ & $\begin{array}{l}2952.706 \\
2954.084\end{array}$ & $\begin{array}{l}33857.35 \\
33841.55\end{array}$ & $a^{4} \mathrm{G}_{41 / 2}-z^{4} \mathrm{G}_{31 / 2}^{0}$ & $-5^{---}$ & .184 & 1.167 & 0.983 & .093 & 1. 811 \\
\hline 1 & 2954.855 & 33832.72 & $e^{4} \mathrm{D}_{31 / 2}-y{ }^{4} \mathrm{G}_{41 / 2}^{0}$ & $\ldots$ & $\ldots+\ldots$ & - 1.10 & $\ldots . . .-$ & $\ldots \ldots$ & $\ldots+\ldots$ \\
\hline 25 & 2960. 216 & 33771.45 & & 4 & 0.180 & 1. 367 & 1. 547 & 0.091 & 0.917 \\
\hline 20 & 2961. 538 & $\begin{array}{l}33756.38 \\
33750.0\end{array}$ & $b{ }^{2} \mathrm{~F}_{21 / 2}-x{ }^{4} \mathrm{G}_{21 / 2}^{\circ}$ & 6 & . 248 & 0.953 & 0.705 & .623 & .830 \\
\hline 60 & 2963. 398 & 33735. 19 & $a^{4} \mathrm{D}_{11 / 2}-z^{4} \mathrm{D}_{01 / 2}^{\circ}$ & 5 & 1. 200 & 1. 200 & .000 & 601 & 1. 800 \\
\hline 200 & 2965.554 & 33710.67 & $a{ }^{4} \mathrm{D}_{21 / 2}-z^{4} \mathrm{~F}_{31 / 2}^{0}$ & 4 & $\ldots \ldots-$ & $(1.337)$ & 1. 28 & $w$ & 1. $14+$ \\
\hline 5 & 2965. 879 & 33706. 98 & $a^{2} \mathrm{I}_{61 / 2}-y^{4} \mathrm{H}_{51 / 2}^{\circ}$ & & & $-\ldots$ & $\ldots \ldots$ & & \\
\hline 10 & 2966. 399 & 33701.07 & - & 5 & $\ldots-\ldots$ & $\ldots-\bar{a}$ - & & $0.18 w$ & 1. $35-$ \\
\hline 10 & 2968. 022 & 33682.64 & $d^{2} \mathrm{D}_{21 / 2}-y^{2} \mathrm{P}_{11 / 2}$ & 4 & (0.08) & 1. 21 & 1. 29 & $.12 w$ & 1. $09+$ \\
\hline $\begin{array}{l}4 h \\
2 h\end{array}$ & 2970. 672 & 33652.59 & $b^{2} \mathrm{D}_{11 / 2}-z^{2} \mathrm{P}_{11 / 2}$ & 6 & 0.21 & $(0.965)$ & 1. 17 & .31 & $?$ \\
\hline & 2971.058 & 33648.23 & $a^{4} \mathrm{G}_{21 / 2}^{1 / 2}-z^{4} \mathrm{G}_{31 / 2}^{\circ}$ & 5 & .333 & 0.645 & 0.978 & .166 & 1. 811 \\
\hline 15 & 2972. 466 & 33632.28 & $b^{4} \mathrm{D}_{21 / 2}-x{ }^{4} \mathrm{D}_{21 / 2}^{\circ}$ & 6 & $(0.07)$ & 1. 35 & 1. 28 & $.17-$ & 1. $32 \pm$ \\
\hline 10 & 2972. 578 & 33631. 02 & $b^{2} \mathrm{G}_{41 / 2}-y^{4} \mathrm{H}_{51 / 2}^{\circ}$ & & & & & - - - - & \\
\hline 8 & 2972.98 & 33626.47 & $b^{2} \mathrm{H}_{51 / 2}-z^{2} \mathrm{I}_{51 / 2}^{\circ}$ & 6 & $(0.03)$ & 1.08 & 1. 05 & $.16-$ & 1. $06 \pm$ \\
\hline $8 h$ & 2973. 826 & 33616.90 & $\left\{\begin{array}{l}b^{2} \mathrm{D}_{21 / 2}-z \\
c^{2}{ }^{2} \mathrm{G}_{312} \mathrm{~F}_{11 / 2}^{11} y^{2} \mathrm{~F}_{0}^{\circ}\end{array}\right.$ & $\ldots-\ldots$ & $\ldots \ldots \ldots$ & $\ldots \ldots$ & $\ldots \ldots$ & $\ldots \ldots$ & $\ldots \ldots$ \\
\hline 4 & 2974. 643 & 33607.67 & $b^{2} \mathrm{~F}_{31 / 2}-y^{4} \mathrm{G}_{31 / 2}^{\circ}$ & 6 & $(0.05)$ & 1. 08 & 1. 03 & $.19-$ & 1. $05 \pm$ \\
\hline 4 & 2974. 882 & 33604.97 & & 4,5 & 0.158 & & & .080 & $?$ \\
\hline 200 & 2976.578 & 33585.82 & $a^{4} \mathrm{D}_{31 / 2}-z{ }^{4} \mathrm{~F}_{41 / 2}^{\circ}$ & 4 & $(0.05)$ & 1. 38 & 1. 33 & $.19 w$ & 1. $14+$ \\
\hline $\begin{array}{l}30 \\
25\end{array}$ & $\begin{array}{l}2977.219 \\
2977.471\end{array}$ & 33578.59 & $a^{4} \mathrm{D}_{01 / 2}-z{ }^{4} \mathrm{~F}_{11 / 2}^{0}$ & 5 & 0.46 & 0.47 & 0.01 & .229 & $0.700 ?$ \\
\hline $\begin{array}{l}25 \\
50\end{array}$ & $\begin{array}{l}2977.471 \\
2978.638\end{array}$ & $\begin{array}{l}33575.75 \\
33562.59\end{array}$ & $\begin{array}{l}b^{2} \mathrm{H}_{51 / 2}-z z^{4} I_{61 / 2}^{0} \\
b^{2} \mathrm{H}_{41 / 2}-z^{2} \mathrm{I}_{51 / 2}^{0}\end{array}$ & $\begin{array}{l}4 ? \\
5\end{array}$ & $\begin{array}{l}(0.04) \\
(0.09)\end{array}$ & $\begin{array}{l}1.08 \\
0.93\end{array}$ & $\begin{array}{l}\text { 1. } 12 \\
\text { 1. } 02\end{array}$ & $\begin{array}{l}.21 w \\
.42 w\end{array}$ & $\begin{array}{l}\text { 1. } 33+ \\
1.45-\end{array}$ \\
\hline 60 & 2979. 713 & 33550.49 & $a{ }^{4} \mathrm{D}_{21 / 2}-z{ }^{4} \mathrm{D}_{21 / 2}^{\circ}$ & 7 & & $(1.337)$ & 1. 331 & 0 & 1. 334 \\
\hline 70 & 2979. 946 & 33547.87 & $a^{4} \mathrm{D}_{11 / 2}-z^{4} \mathrm{~F}_{21 / 2}^{072}$ & 4 & 0.14 & 1. 26 & 1. 12 & $0.22 w$ & $0.90+$ \\
\hline 25 & 2980. 966 & 33536. 39 & $b^{2} \mathrm{G}_{41 / 2}-x{ }^{4} \mathrm{G}_{31 / 2}$ & 5 & $(0.08)$ & 1.09 & 1. 01 & $.27 w$ & 1. $36-?$ \\
\hline $10 H$ & $\begin{array}{l}2982.026 \\
2983.781\end{array}$ & 33524. 47 & $b{ }^{2} \mathrm{P}_{01 / 2}-x^{4} \mathrm{~F}_{11 / 2}$ & $\ldots$ & $\ldots \ldots$ & $\ldots \ldots$ & $\ldots \ldots$ & $\ldots \ldots$ & $\ldots \ldots$ \\
\hline & 2983. 781 & 33504.75 & $a{ }^{4} \mathrm{G}_{21 / 2}-y{ }^{4} \mathrm{P}_{21 / 2}^{0}$ & $\ldots \ldots$ & $\ldots \ldots$ & $\ldots \ldots$ & $\ldots \ldots$ & $\ldots+\cdots$ & $\ldots \ldots$ \\
\hline
\end{tabular}


TABLE 6. The second spectrum of ruthenium (Ru II) - Continued

\begin{tabular}{|c|c|c|c|c|c|c|c|c|c|}
\hline $\begin{array}{c}1 \\
\text { Intensity }\end{array}$ & $\begin{array}{c}2 \\
\lambda(\text { air })\end{array}$ & $\begin{array}{c}3 \\
\sigma\end{array}$ & $\begin{array}{c}4 \\
\text { Term combina- } \\
\text { tion }\end{array}$ & $\begin{array}{c}5 \\
\text { Type }\end{array}$ & $\begin{array}{c}6 \\
\Delta g\end{array}$ & 7 & $\begin{array}{l}8 \\
g\end{array}$ & $\begin{array}{c}9 \\
\text { Strong } p\end{array}$ & $\begin{array}{c}10 \\
\text { Strong } n\end{array}$ \\
\hline & $A$ & $K$ & & & & & & & \\
\hline $\begin{array}{l}5 h \\
10 \\
70 \\
15 \\
20 h\end{array}$ & $\begin{array}{l}\text { 2985. } 676 \\
\text { 2991. } 453 \\
\text { 2991. } 621 \\
\text { 2992. } 083 \\
\text { 2992. } 601\end{array}$ & $\begin{array}{l}33483.49 \\
33418.82 \\
33416.95 \\
33411.79 \\
33406.01\end{array}$ & 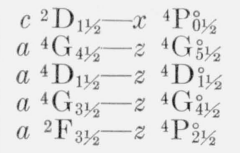 & $\begin{array}{r}4 \\
5 \\
7 \\
- \\
-4\end{array}$ & $\begin{array}{c}0.940 \\
(0.06) \\
0.440\end{array}$ & $\begin{array}{l}0.809 \\
1.18 \\
(1.188) \\
(1.14)\end{array}$ & $\begin{array}{l}\text { 1. } 749 \\
\text { 1. } 24 \\
\text { 1. } 156\end{array}$ & $0^{.28 w}$ & $\begin{array}{l}0.339 \\
\text { 1. } 52- \\
\text { 1. } 172 \\
\\
?\end{array}$ \\
\hline $\begin{array}{c}10 \\
5 h \\
20 h \\
1 \\
3 h\end{array}$ & $\begin{array}{l}\text { 2993. } 142 \\
\text { 2994. } 650 \\
\text { 2996. } 002 \\
\text { 2996. } 331 \\
\text { 2998. } 126\end{array}$ & $\begin{array}{l}\text { 33399. } 97 \\
33383.15 \\
33368.09 \\
33364.42 \\
33344.45\end{array}$ & $\begin{array}{lll}b & { }^{2} \mathrm{D}_{21 / 2}-x & { }^{4} \mathrm{D}_{31 / 2}^{\circ} \\
a & { }^{2} \mathrm{I}_{51 / 2}-y & { }^{4} \mathrm{H}_{41 / 2}^{\circ} \\
b & { }^{2} \mathrm{P}_{11 / 2}-x & { }^{4} \mathrm{D}_{11 / 2}^{\circ} \\
b & { }^{2} \mathrm{P}_{11 / 2}-z & { }^{2} \mathrm{~F}_{21 / 2}^{\circ} \\
b & { }^{2} \mathrm{P}_{01 / 2}-z & { }^{2} \mathrm{P}_{01 / 2}^{\circ}\end{array}$ & 7,6 & 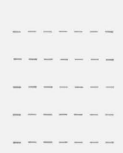 & (1. 188$)$ & 1. 21 & 0 & 1. 20 \\
\hline 75 & 2998. 886 & 33336. 00 & $a^{4} \mathrm{D}_{01 / 2}-z{ }^{4} \mathrm{D}_{01 / 2}^{\circ}$ & Una & fected & & & & \\
\hline & $\begin{array}{l}\text { 2999. } 789 \\
\text { 3000. } 465\end{array}$ & $\begin{array}{l}33325.96 \\
33318.46\end{array}$ & 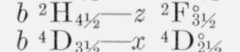 & 4 & 0. 248 & 0.960 & 1. 208 & 0.124 & 0. 092 \\
\hline $\begin{array}{r}25 \\
1\end{array}$ & $\begin{array}{l}3005.141 \\
3005.891\end{array}$ & $\begin{array}{l}33266.61 \\
33258.31\end{array}$ & $\begin{array}{l}c^{2} \mathrm{D}_{21 / 2}-x^{2} \mathrm{~F}_{31 / 2}^{21 / 2} \\
c^{2} \mathrm{D}_{11 / 2}-w^{4} \mathrm{~F}_{21 / 2}^{\circ}\end{array}$ & 4 & - & 1. 17 & (1. 137) & $w$ & 1. $05+$ \\
\hline $\begin{array}{l}10 \\
2 h\end{array}$ & $\begin{array}{l}\text { 3006. } 716 \\
\text { 3007. } 88\end{array}$ & $\begin{array}{l}\text { 33249. } 19 \\
33236.32\end{array}$ & $a^{2} \mathrm{~F}_{21}-z^{4} \mathrm{~F}_{21}$ & $\begin{array}{l}7 \\
6\end{array}$ & 21 & & $(1.07)$ & 0 & 1. 27 \\
\hline & $\begin{array}{l}3010.450 \\
3014.360\end{array}$ & $\begin{array}{l}33207.95 \\
33164.88\end{array}$ & 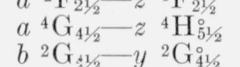 & $\begin{array}{l}0 \\
4\end{array}$ & $(0.09)$ & $\begin{array}{l}0.86 \\
1.13\end{array}$ & 1. 04 & $\begin{array}{l}0.105 \\
.40 w\end{array}$ & $0 . \stackrel{?}{6} 4+$ \\
\hline 30 & 3015.410 & 33153.33 & $\left\{\begin{array}{lll}b & 2 \mathrm{H}_{51 / 2}-z & { }^{2} \mathrm{G}_{41 / 2} \\
b & { }^{2} \mathrm{D}_{11 / 2}-y & { }^{4} \mathrm{G}_{21 / 2}^{1}\end{array}\right.$ & 5 & $(0.06)$ & 1. 05 & 0. 99 & $0.29 w$ & 1. $32-$ \\
\hline 5 & 3015. 956 & 33147. 33 & $b{ }^{2} \mathrm{~F}_{21 / 2}-z{ }^{2} \mathrm{P}_{11 / 2}^{0}$ & 4 & 0. 200 & 0.951 & 1. 151 & .100 & 0.651 \\
\hline 30 & 3017. 816 & 33126. 90 & $b^{2} \mathrm{~F}_{31 / 2}-y^{4} \mathrm{G}_{41 / 2}^{\circ}$ & 5 & $(0.06)$ & 1. 09 & 1. 15 & $.22 w$ & 1. $37-$ \\
\hline $\begin{array}{l}1 h \\
1\end{array}$ & $\begin{array}{l}3018.690 \\
3019.642\end{array}$ & $\begin{array}{l}\text { 33117. } 31 \\
33106.87\end{array}$ & 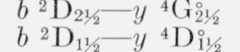 & $\cdots$ & $\cdots \cdots$ & $\ldots-\ldots$ & 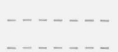 & $\cdots$ & $\cdots$ \\
\hline 5 & 3019. 76 & 33105.57 & $a^{2} \mathrm{~F}_{21 / 2}-z{ }^{4} \mathrm{D}_{11 / 2}^{1 / 2}$ & 4 & 0.270 & 0.871 & 1. 141 & .134 & 0. 466 \\
\hline 1 & 3020.549 & 33096. 92 & $b^{2} \mathrm{G}_{31 / 2}-y{ }^{4} \mathrm{H}_{41 / 2}$ & & $\ldots \ldots$ & $\ldots \ldots$ & $\ldots-\ldots$ & $\ldots \ldots-$ & $\ldots \ldots$ \\
\hline $1 h$ & 3021.23 & 33089.47 & 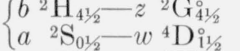 & \}- & $\ldots \ldots$ & $\cdots-$ & & $\ldots \ldots$ & \\
\hline $\begin{array}{r}1 h \\
10 h\end{array}$ & $\begin{array}{l}\text { 3021. } 977 \\
3022.61\end{array}$ & $\begin{array}{l}33081.29 \\
33074.36\end{array}$ & $a^{2}{ }^{2} \mathrm{I}_{51 / 2}-y{ }^{4} \mathrm{H}_{61 / 2}$ & & $\ldots$ & & & & \\
\hline $\begin{array}{l}10 h \\
10 h\end{array}$ & $\begin{array}{l}3022.61 \\
3022.946\end{array}$ & $\begin{array}{l}33074.36 \\
33070.68\end{array}$ & $\begin{array}{l}c^{2} \mathrm{D}_{11 / 2}-2_{11 / 2}^{\mathrm{i}} \\
b^{2} \mathrm{D}_{21 / 2}-y^{4} \mathrm{D}_{11 / 2}^{\circ}\end{array}$ & $\begin{array}{l}6 \\
4\end{array}$ & 0.133 & $\begin{array}{l}\text { (0. } 789) \\
\text { 1. } 106\end{array}$ & $\begin{array}{l}0.85 \\
\text { 1. } 239\end{array}$ & 0. $\stackrel{w}{066}$ & $\begin{array}{r}0.82 \\
.91\end{array}$ \\
\hline $\begin{array}{l}8 \\
8 h\end{array}$ & $\begin{array}{l}\text { 3023. } 841 \\
\text { 3026. } 817\end{array}$ & $\begin{array}{l}33060.90 \\
33028.39\end{array}$ & $\begin{array}{l}b^{2}{ }^{2} \mathrm{~F}_{31 / 2}-z z^{2} \mathrm{~F}_{21 / 2}^{\circ} \\
c^{2} \mathrm{D}_{21 / 2}-y{ }^{2} \mathrm{~F}_{311}^{o}\end{array}$ & 5 & .232 & 1. 087 & 0.855 & .117 & 1. 667 \\
\hline $\begin{array}{r}30 \\
1\end{array}$ & $\begin{array}{l}\text { 3027. } 780 \\
\text { 3028. } 271\end{array}$ & $\begin{array}{l}33017.88 \\
33012.53\end{array}$ & $a{ }^{4} \mathrm{D}_{01 / 2}-z{ }^{4} \mathrm{D}_{11 / 2}^{0}$ & 5 & 1. 144 & 0. 000 & 1. 444 & .573 & 1. 716 \\
\hline 5 & 3028.928 & 33005.37 & $b^{4} \mathrm{D}_{31 / 2}-y{ }^{4} \mathrm{D}_{31 / 2}^{\circ}$ & 6 & $(0.04)$ & 1. 38 & 1. 34 & $.13-$ & 1. $36 \pm$ \\
\hline 5 & 3032. 250 & 32969. 21 & $a{ }^{2} \mathrm{~S}_{01 / 2}-x{ }^{4} \mathrm{P}_{01 / 2}^{\circ}$ & & & & & & \\
\hline $\begin{array}{l}7 \\
5\end{array}$ & $\begin{array}{l}\text { 3032. } 442 \\
\text { 3032. } 624\end{array}$ & $\begin{array}{l}\text { 32967. } 13 \\
32965.15\end{array}$ & $b^{2} \mathrm{~F}_{21 / 2}-y{ }^{2} \mathrm{G}_{31 / 2}^{0}$ & 5 & $(0.06)$ & 0.99 & 1. 05 & $.15 w$ & 1. $20-$ \\
\hline 8 & 3033. 911 & 32951. 16 & $a^{2} \mathrm{I}_{51 / 2}-y^{2} \mathrm{G}_{41 / 2}$ & - & $-\ldots-n$ & $\ldots-\cdots$ & - & n-n & $n_{-n-1}$ \\
\hline $15 h$ & 3035. 792 & 32930.75 & $b{ }^{2} \mathrm{~F}_{21 / 2}-x{ }^{4} \mathrm{D}_{31 / 2}^{0}$ & 7 & $\ldots \ldots \ldots$ & $(0.945)$ & 0.95 & 0 & 0. 967 \\
\hline $\begin{array}{l}75 \\
2 h\end{array}$ & $\begin{array}{l}\text { 3036. } 463 \\
\text { 3037. } 116\end{array}$ & $\begin{array}{l}32923.47 \\
32916.40\end{array}$ & $\begin{array}{l}b^{2}{ }^{2} \mathrm{H}_{51 / 2}-z{ }^{2} \mathrm{I}_{61 / 2}^{\circ} \\
a{ }^{6} \mathrm{~S}_{21 / 2}-y^{4} \mathrm{D}_{21 / 2}^{\circ}\end{array}$ & 5 & $(0.07)$ & 1. 08 & 1. 15 & $0.36 w$ & 1. $51-$ \\
\hline $\begin{array}{l}15 \\
1 h\end{array}$ & $\begin{array}{l}\text { 3038. } 758 \\
3039.50\end{array}$ & $\begin{array}{l}32898.61 \\
32890.58\end{array}$ & 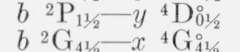 & 5 & 0. 410 & 1. 187 & 0.777 & .205 & 1. 392 \\
\hline 2 & 3040.096 & 32884.13 & $c^{2} \mathrm{D}_{21 / 2}-x{ }^{4} \mathrm{P}_{11 / 2}$ & & $\ldots-\ldots$ & - & - & $\ldots$ & - n \\
\hline $\begin{array}{l}1 \\
1 h\end{array}$ & $\begin{array}{l}3042.367 \\
3043.342\end{array}$ & $\begin{array}{l}32859.58 \\
32849.06\end{array}$ & $\begin{array}{l}z^{4} \mathrm{D}_{11 / 2}^{\circ}-e{ }^{4} \mathrm{D}_{21 / 2} \\
b^{2} \mathrm{H}_{51 / 2}-z \\
{ }^{4} \mathrm{I}_{51 / 2}^{\circ}\end{array}$ & & & & & & \\
\hline 10 & $\begin{array}{l}3046.132 \\
3046.827\end{array}$ & $\begin{array}{l}32818.97 \\
32811.49\end{array}$ & $a^{4} \mathrm{D}_{11 / 2}-z{ }^{4} \mathrm{D}_{21 / 2}^{2}$ & 5 & .155 & 1. 193 & 1. 348 & .077 & 1. 580 \\
\hline $\begin{array}{l}\text { lh } \\
1\end{array}$ & $\begin{array}{l}3046.827 \\
3047.223\end{array}$ & $\begin{array}{l}32811.49 \\
32807.22\end{array}$ & $\begin{array}{l}c^{2} \mathrm{D}_{11 / 2}-w^{4} \mathrm{~F}^{11 / 2} \\
a^{4} \mathrm{G}_{41 / 2}-z^{4} \mathrm{H}_{41 / 2}^{\circ}\end{array}$ & & $\ldots \ldots$ & $\ldots-\cdots$ & $-\cdots--$ & - & $\ldots$ \\
\hline 25 & 3047. 702 & 32802. 07 & $a^{4} \mathrm{D}_{21 / 2}-z{ }^{4} \mathrm{D}_{31 / 2}^{\circ}$ & 5 & & (1. 337) & 1. 38 & w & 1. $50-$ \\
\hline $\begin{array}{r}50 \\
1\end{array}$ & $\begin{array}{l}\text { 3049. } 246 \\
3053.80\end{array}$ & $\begin{array}{l}32785.46 \\
32736.57\end{array}$ & $b{ }^{2} \mathrm{H}_{41 / 2}-z{ }^{4} \mathrm{I}_{51 / 2}^{\circ}$ & 5 & $(0.08)$ & 0.98 & 1. 06 & $0.38 w$ & 1. $44-$ \\
\hline $5 H$ & 3054.563 & 32728. 39 & $z^{4} \mathrm{~F}_{21 / 2}^{0}-e^{4} \mathrm{D}_{21 / 2}$ & & 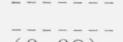 & - & & & \\
\hline 100 & 3056.855 & 32703.85 & $b^{2} \mathrm{H}_{51 / 2}-y{ }^{4} \mathrm{~F}_{41 / 2}^{\circ}$ & 4 & $(0.08)$ & 1. 07 & 1. 15 & $0.35 w$ & $0.72+$ \\
\hline
\end{tabular}


TABLE 6. The second spectrum of ruthenium (Ru II) - Continued

\begin{tabular}{|c|c|c|c|c|c|c|c|c|c|}
\hline $\begin{array}{c}1 \\
\text { Intensity }\end{array}$ & $\begin{array}{c}2 \\
\lambda(\text { air })\end{array}$ & $\begin{array}{l}3 \\
\sigma\end{array}$ & $\begin{array}{c}4 \\
\text { Term combina- } \\
\text { tion }\end{array}$ & $\begin{array}{c}5 \\
\text { Type }\end{array}$ & $\begin{array}{c}6 \\
\Delta g\end{array}$ & $\begin{array}{l}7 \\
g\end{array}$ & 8 & $\begin{array}{c}9 \\
\text { Strong } p\end{array}$ & $\begin{array}{c}10 \\
\text { Strong } n\end{array}$ \\
\hline & $A$ & $K$ & & & & & & & \\
\hline 20 & 3059.101 & 32679.84 & $b^{4} \mathrm{D}_{31 / 2}-z^{2} \mathrm{~F}_{31 / 2}^{\circ}$ & & & & & & \\
\hline 30 & 3060. 217 & 32667.93 & $a^{2} \mathrm{~F}_{21 / 2}^{0}-z{ }^{4} \mathrm{~F}_{31 / 2}^{\circ}$ & 5 & 0. 416 & 0.846 & 1. 262 & 209 & 2. 302 \\
\hline 30 & 3060.500 & 32664.90 & $b^{2} \mathrm{G}_{31 / 2}-y^{2} \mathrm{G}_{41 / 2}$ & 5 & 179 & .869 & 1. 048 & 089 & 1. $67 ?$ \\
\hline $8 h$ & 3062.07 & 32648. 16 & $b{ }^{2} \mathrm{~F}_{21 / 2}-y{ }^{4} \mathrm{G}_{21 / 2}$ & 6 & .260 & .942 & 0. 682 & 651 & 0.812 \\
\hline 1 & 3062. \&19 & 32640.17 & $b^{2} \mathrm{H}_{41 / 2}-y{ }^{4} \mathrm{~F}_{41 / 2}^{0}$ & -..- & $\ldots \ldots$ & $\ldots \ldots$ & $\ldots \ldots$ & $\ldots-\ldots$ & $\ldots \ldots \ldots$ \\
\hline 1 & 3064. 366 & 32623. 69 & & & & & & & \\
\hline 20 & 3066. 435 & 32601. 69 & b ${ }^{2} \mathrm{~F}_{21 / 2}-y{ }^{4} \mathrm{D}_{11 / 2}^{\circ}$ & 4 & .278 & .954 & 1. 232 & .139 & .537 \\
\hline $\begin{array}{l}3 \\
4\end{array}$ & $\begin{array}{l}3067.575 \\
3070.317\end{array}$ & $\begin{array}{l}32589.57 \\
32560.47\end{array}$ & $c{ }^{2} \mathrm{G}_{31 / 2}-y^{2} \mathrm{D}_{21 / 2}^{\circ}$ & $\ldots$ & $\ldots \ldots$ & $\ldots \ldots$ & $\ldots \ldots$ & $\ldots \ldots$ & $\ldots \ldots$ \\
\hline 30 & 3072. 341 & 32539. 02 & $b^{2} \mathrm{G}_{31 / 2}-x{ }^{4} \mathrm{G}_{21 / 2}^{\circ}$ & 5 & 186 & .880 & 0.694 & .093 & 1. 345 \\
\hline 40 & 3073. 548 & 32526. 24 & $b^{2} \mathrm{H}_{41 / 2}-z^{2} \mathrm{G}_{31 / 2}^{0}$ & 4 & $(0.07)$ & .92 & .99 & $.26 w$ & $0.66+$ \\
\hline 20 & 3075.33 & 32507.39 & $a^{2} \mathrm{~F}_{21 / 2}-z^{4} \mathrm{D}_{21 / 2}^{0}$ & 6 & 0.475 & .873 & 1. 348 & 1. 191 & 1. 111 \\
\hline 4 & 3079. 120 & 32467.38 & $a^{4} \mathrm{G}_{31 / 2}-z{ }^{4} \mathrm{H}_{41 / 2}^{0}$ & 7 & $\ldots \ldots-$ & (1.021) & 1. 026 & 0 & 1. 043 \\
\hline 25 & 3081. 396 & 32443. 40 & $b^{4} \mathrm{D}_{31 / 2}-z^{2} \mathrm{G}_{41 / 2}^{0}$ & 4 & .391 & 1. 384 & 0.993 & -0.197 & -0.386 \\
\hline 10 & 3081.847 & 32438.65 & $a^{4} \mathrm{D}_{11 / 2}-z{ }^{6} \mathrm{P}_{1 / 2}^{1 / 12}$ & $-\cdots$ & $\ldots \ldots$ & $\ldots \ldots$ & $\ldots \ldots$ & $-\ldots-\ldots$ & $\ldots \ldots \ldots$ \\
\hline 1 & 3082. 963 & 32426. 91 & $a^{4} \mathrm{G}_{41 / 2}-z{ }^{4} \mathrm{H}_{31 / 2}^{\circ}$ & & & & & & $\ldots \ldots-\ldots$ \\
\hline 25 & 3086. 624 & 32388.45 & a ${ }^{2} \mathrm{I}_{61 / 2}-x^{4} \mathrm{G}_{51 / 2}^{0}$ & 4 & $(0.07)$ & 1. 04 & 1. 11 & $.40 w$ & $.64+$ \\
\hline $\begin{array}{r}1 \\
10\end{array}$ & $\begin{array}{l}3087.924 \\
3089689\end{array}$ & $\begin{array}{l}32374.82 \\
32356.32\end{array}$ & $z^{4} \mathrm{D}_{21 / 2}^{\circ}-e{ }^{4} \mathrm{D}_{31 / 2}$ & $-\cdots$ & $\ldots \ldots$ & $\ldots+\cdots$ & $\ldots \ldots$ & $-\ldots-\ldots$ & $\ldots-\ldots$ \\
\hline 5 & 3090.523 & 32347.59 & 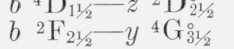 & 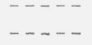 & - n & - n & - n & - & - \\
\hline 100 & 3093. 902 & 32312. 26 & $b^{2} \mathrm{G}_{41 / 2}-x{ }^{4} \mathrm{G}_{51 / 2}^{0}$ & 5 & $(0.08)$ & 1. 06 & 1. 14 & $.34 w$ & 1. $48-$ \\
\hline 25 & 3094.565 & 32305.34 & $a^{4} \mathrm{D}_{21 / 2}-z{ }^{6} \mathrm{P}_{21 / 2}$ & 6 & 0.500 & 1. 340 & 1. 840 & 1. 248 & 1. 591 \\
\hline 20 & 3097. 960 & 32269. 94 & $b^{2} \mathrm{D}_{21 / 2}-z^{2} \mathrm{~F}_{21 / 2}^{0}$ & 6 & .26 & (1. 102) & 0.84 & 0.65 & $?$ \\
\hline $\begin{array}{l}3 \\
2 h\end{array}$ & $\begin{array}{l}3099.908 \\
3101.462\end{array}$ & $\begin{array}{l}32249.66 \\
32233.51\end{array}$ & $\begin{array}{l}b^{2}{ }^{2} \mathrm{G}_{41}-y{ }^{2} \mathrm{G}_{31 / 2}^{8} \\
a^{4} \mathrm{G}^{4}\end{array}$ & $\cdots-$ & $\ldots \ldots$ & $\ldots+\cdots$ & $-\ldots-\cdots$ & $-\ldots-\cdots$ & 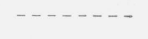 \\
\hline & & & & & & & & & \\
\hline $1 h$ & 3102. 532 & 32222. 39 & $c{ }^{2} \mathrm{G}_{41 / 2}-y{ }^{4} \mathrm{H}_{31 / 2}^{\circ}$ & -7 & & & & & \\
\hline 30 & 3103. 422 & 32213. 15 & $b{ }^{2} \mathrm{G}_{41 / 2}-x{ }^{4} \mathrm{D}_{31 / 2}^{0}$ & 5 & 0. 116 & 1. 067 & 0.951 & 0.058 & 1. 473 \\
\hline 30 & 3107.586 & 32169. 99 & $a^{4} \mathrm{D}_{31 / 2}-z{ }^{6} \mathrm{P}_{31 / 2}^{0}$ & 6 & .178 & 1. 407 & 1. 585 & .626 & 1. 496 \\
\hline $\begin{array}{l}5 n \\
10 H\end{array}$ & $\begin{array}{l}3111.148 \\
3113.947\end{array}$ & $\begin{array}{l}32133.16 \\
32104.28\end{array}$ & 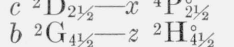 & $\begin{array}{l}6 \\
6\end{array}$ & $\begin{array}{l}(0.07) \\
(0.06)\end{array}$ & $\begin{array}{l}1.18 \\
1.07\end{array}$ & $\begin{array}{l}\text { (1. } 246) \\
1.01\end{array}$ & $\begin{array}{l}.18- \\
.26-\end{array}$ & 1. $\stackrel{?}{04+}$ \\
\hline $5 h$ & 3115. 606 & 32087.18 & $a{ }^{4} \mathrm{G}_{31 / 2}-z{ }^{4} \mathrm{H}_{31 / 2}^{0}$ & & & & & & \\
\hline $20 ?$ & 3118.03 & 32062. 2 & $b^{2} \mathrm{H}_{41 / 2}^{0}-y{ }^{4} \mathrm{~F}_{31 / 2}^{0}$ & 4 & 0.10 & 0.93 & 1. 03 & $.36 w$ & $0.58+$ \\
\hline 3 & 3120. 234 & 32039. 59 & $a^{4} \mathrm{D}_{01 / 2}-z{ }^{6} \mathrm{P}_{11 / 2}$ & $-\overline{6}$ & -780 & 200 & 0.420 & 169 & 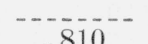 \\
\hline $\begin{array}{l}25 \\
10\end{array}$ & $\begin{array}{l}3120.872 \\
3123.892\end{array}$ & $\begin{array}{l}32033.04 \\
32002.07\end{array}$ & $\begin{array}{llll}b & { }^{4} \mathrm{D}_{11 / 2}-y & { }^{4} & { }^{11 / 2} \\
b & { }^{2} \mathrm{P}_{01 / 2}-x & { }^{4} \mathrm{D}_{01 / 2}^{\circ}\end{array}$ & $\begin{array}{l}6 \\
6\end{array}$ & $\begin{array}{l}.780 \\
.51\end{array}$ & $\begin{array}{l}1.200 \\
0.88\end{array}$ & $\begin{array}{l}0.420 \\
.37\end{array}$ & $\begin{array}{l}\text { 1. } 109 \\
0.254\end{array}$ & $\begin{array}{l}.810 \\
.627\end{array}$ \\
\hline 25 & 3124. 678 & 31994. 02 & $b{ }^{4} \mathrm{D}_{31 / 2}-y{ }^{4} \mathrm{~F}_{41 / 2}^{0}$ & & $-\ldots-$. & & & & \\
\hline 20 & 3126.613 & 31974. 22 & $b{ }^{2} \mathrm{~F}_{31 / 2}-z{ }^{2} \mathrm{~F}_{31 / 2}^{\circ}$ & 6 & .116 & 1. 082 & 1. 198 & .415 & 1. 140 \\
\hline 25 & 3127.923 & 31960.83 & $b{ }^{4} \mathrm{D}_{01 / 2}-y{ }^{4} \mathrm{~F}_{11 / 2}^{0}$ & 5 & 298 & 0.139 & 0.437 & .149 & 0.586 \\
\hline 10 & 3132.701 & 31912. 09 & $b^{4} \mathrm{D}_{31 / 2}-z{ }^{2} \mathrm{D}_{21 / 2}^{0}$ & 5 & $(0.06)$ & 1. 40 & 1. 34 & $.15 \mathrm{w}$ & 1. $55-$ \\
\hline 25 & 3134.817 & 31890.55 & $a^{2} \mathrm{I}_{51 / 2}-z^{2} \mathrm{H}_{41 / 2}^{0}$ & 4 & $(0.08)$ & 0.94 & 1. 02 & $.37 w$ & $0.57+$ \\
\hline 10 & 3135. 199 & 31886.66 & $c^{2} \mathrm{D}_{11 / 2}-y^{2} \mathrm{~F}_{21 / 2}^{\circ}$ & & & & & & \\
\hline 25 & 3135. 807 & 31880.48 & $b^{4} \mathrm{D}_{31 / 2}-z^{2} \mathrm{G}_{31 / 2}^{0}$ & 6 & 0.362 & 1. 391 & 1. 029 & 1. 270 & 1. 210 \\
\hline 1 & 3138. 022 & 31857. 98 & $b{ }^{2} \mathrm{D}_{11 / 2}-x{ }^{4} \mathrm{D}_{21 / 2}$ & 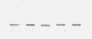 & $\ldots \ldots$ & $\ldots-\ldots$ & $\ldots \ldots \ldots$ & $\ldots \ldots-\ldots$ & - $-\ldots-\ldots$ \\
\hline $\begin{array}{r}2 \\
10\end{array}$ & $\begin{array}{l}3139.576 \\
3141.594\end{array}$ & 31842. 21 & $d^{2} \mathrm{D}_{11 / 2}-x{ }^{2} \mathrm{D}_{11 / 2}$ & --- & 180 & 1097 & $1277^{-}$ & 0453 & $187^{-}$ \\
\hline & & & & & & & & & 141 \\
\hline $\begin{array}{l}40 \\
60\end{array}$ & 3143.240 & 31805.09 & 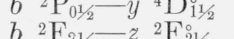 & $\begin{array}{l}5 \\
6\end{array}$ & $\begin{array}{l}.361 \\
12\end{array}$ & $\begin{array}{l}0.868 \\
0.99\end{array}$ & 1. 230 & $\cdot \begin{array}{l}181 \\
20\end{array}$ & 1. 411 \\
\hline 15 & 3146. 09 & $\begin{array}{l}31800.94 \\
31776.28\end{array}$ & $a^{2} \mathrm{~F}_{31 / 2}^{21 / 2} z{ }^{4} \mathrm{D}_{21 / 2}^{21 / 2}$ & 4 & 206 & 1. 139 & 1. 345 & .103 & $\begin{array}{c}0.93 \pm \\
.624\end{array}$ \\
\hline 3 & 3146. 395 & 31773. 20 & $b{ }^{2} \mathrm{P}_{11 / 2}-y{ }^{4} \mathrm{P}_{11 / 2}^{0}$ & $-\ldots$ & $\cdots-\cdots$ & & & & \\
\hline 50 & 3147.456 & 31762. 49 & $b^{2} \mathrm{H}_{51 / 2}^{0}-z{ }^{4} \mathrm{I}_{41 / 2}$ & 5 & .120 & 1. 06 & 0.94 & .06 & 1. $60-$ \\
\hline 2 & 3148. 716 & 31749. 78 & $b^{2} \mathrm{G}_{31 / 2}-y{ }^{2} \mathrm{G}_{31 / 2}^{\circ}$ & $\ldots \ldots$ & $\ldots \ldots$ & $\ldots \ldots \ldots$ & $-\ldots$ & $-\ldots-\ldots$ & $\ldots-\ldots$ \\
\hline 3 & 3148.913 & 31747. 80 & $c^{2} \mathrm{G}_{41 / 2}-y{ }^{2} \mathrm{G}_{41 / 2}^{0}$ & $\ldots-\ldots$ & $-\ldots-\ldots$ & $\ldots-\ldots-$ & $\ldots \ldots$ & $\ldots \ldots-\ldots$ & $\ldots-\ldots$ \\
\hline 1 & 3149. 901 & 31737.84 & $b^{2} \mathrm{~F}_{31 / 2}-z{ }^{2} \mathrm{G}_{41 / 2}$ & ..... & $\ldots \ldots$ & $\ldots \ldots$ & $\ldots \ldots-\cdots$ & ------ & $\ldots \ldots \ldots$ \\
\hline $\begin{array}{l}2 \\
2\end{array}$ & $\begin{array}{l}3152.311 \\
3157.173\end{array}$ & $\begin{array}{l}31713.57 \\
31664.74\end{array}$ & 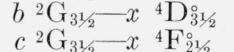 & $\cdots-$ & 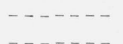 & 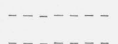 & 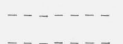 & $---1--$ & 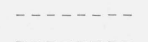 \\
\hline
\end{tabular}


TABLE 6. The second spectrum of ruthenium (Ru II) - Continued

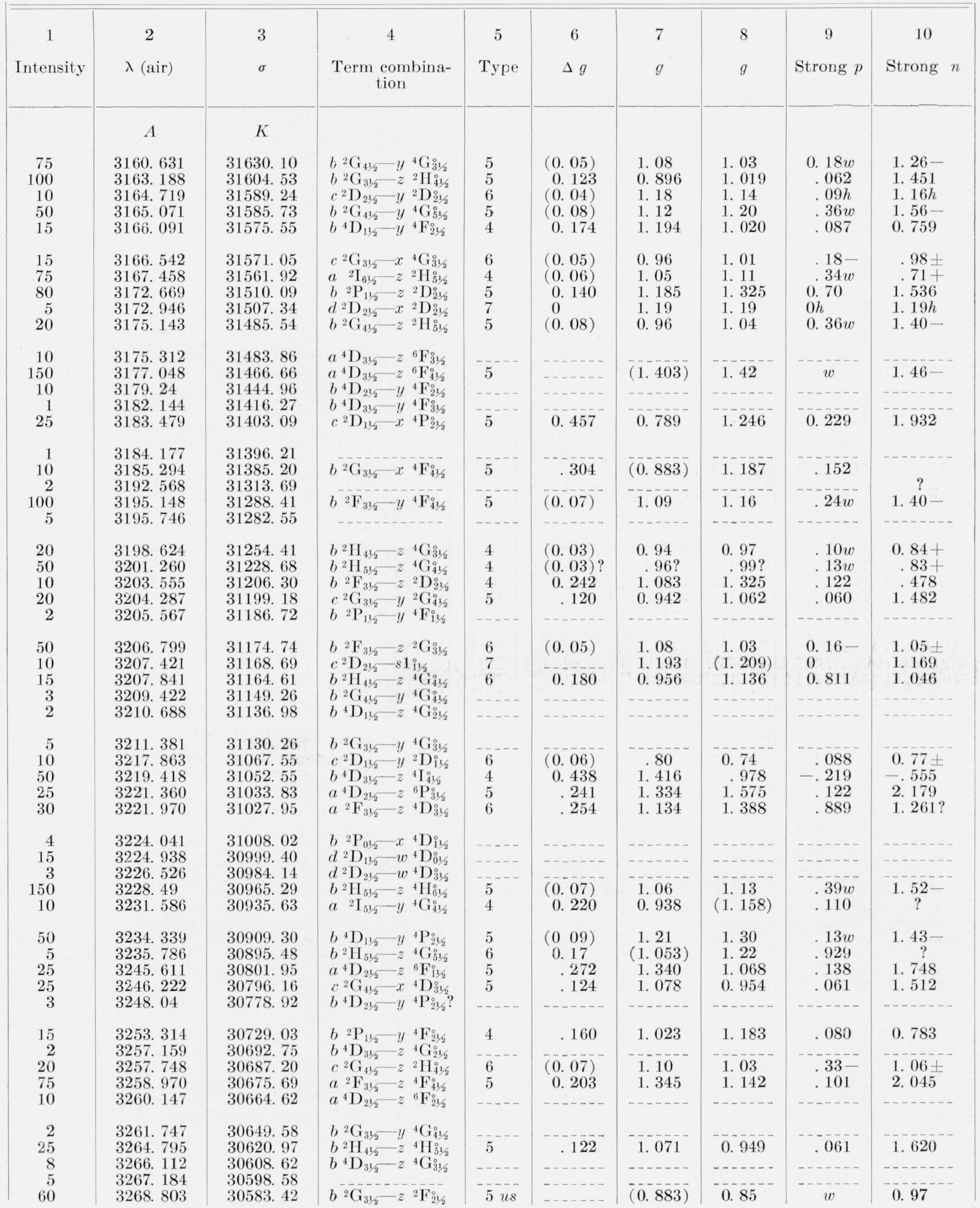


TABLE 6. The second spectrum of ruthenium (Ru II)-Continued

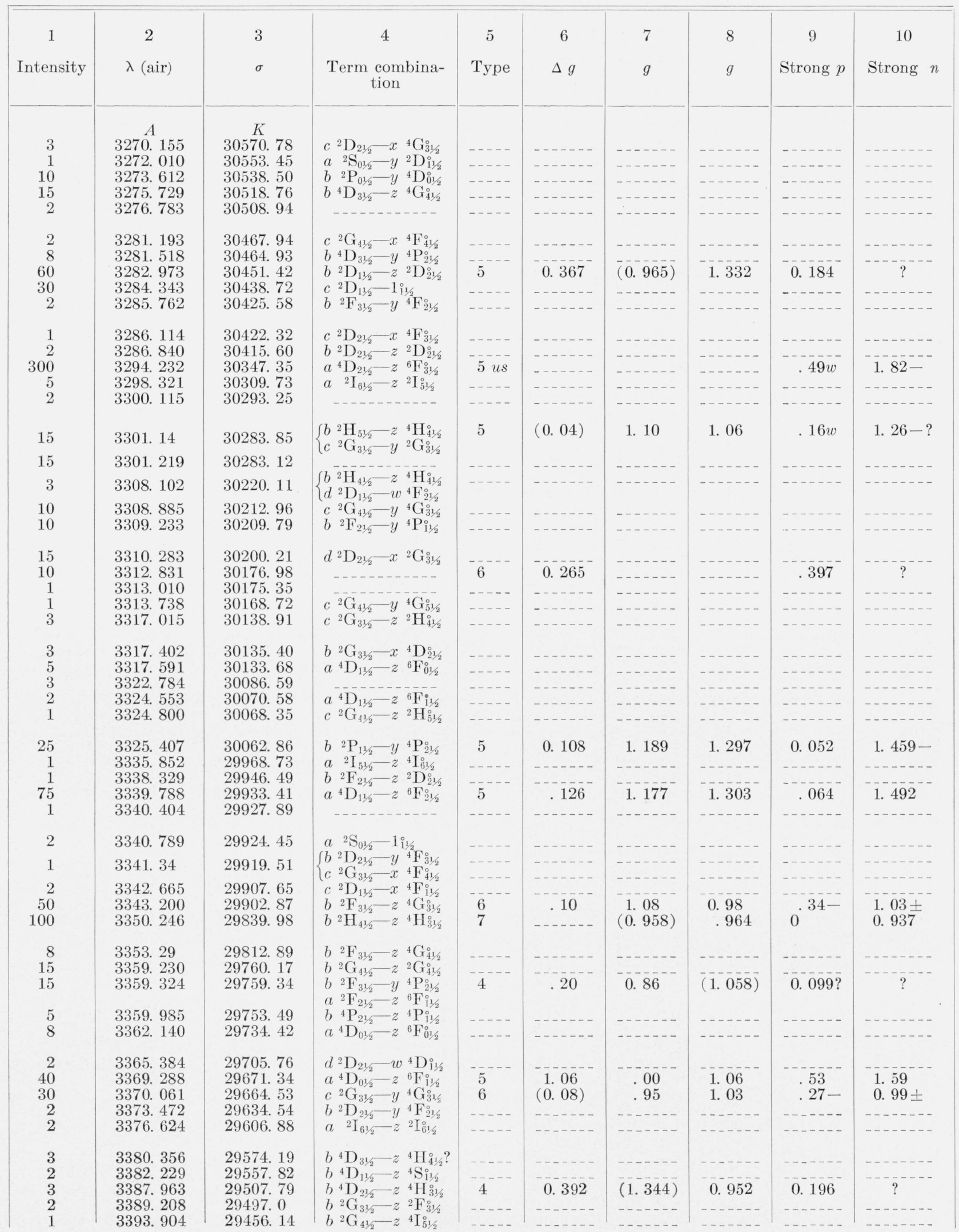


TABLE 6. The second spectrum of ruthenium (Ru II)-Continued

\begin{tabular}{|c|c|c|c|c|c|c|c|c|c|}
\hline $\begin{array}{c}1 \\
\text { Intensity }\end{array}$ & $\begin{array}{c}2 \\
\lambda \text { (air) }\end{array}$ & $\begin{array}{l}3 \\
\sigma\end{array}$ & $\begin{array}{c}4 \\
\text { Term combina- } \\
\text { tion }\end{array}$ & $\begin{array}{c}5 \\
\text { Type }\end{array}$ & $\begin{array}{c}6 \\
\Delta g\end{array}$ & $\begin{array}{l}7 \\
g\end{array}$ & $\begin{array}{l}8 \\
g\end{array}$ & $\begin{array}{c}9 \\
\text { Strong } p\end{array}$ & $\begin{array}{c}10 \\
\text { Strong } n\end{array}$ \\
\hline & $A$ & $K$ & & & & & & & \\
\hline 8 & 3398. 862 & 29413. 17 & $b{ }^{2} \mathrm{P}_{01 / 2}-y{ }^{4} \mathrm{P}_{11 / 2}^{\circ}$ & $5 u s$ & $\ldots \ldots$ & & 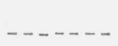 & .84 & 1. 58 \\
\hline 5 & 3401. 188 & 29393. 06 & $a{ }^{2} \mathrm{~S}_{01 / 2}-x{ }^{4} \mathrm{~F}_{11 / 2}$ ? & $\ldots .-$ & $\ldots \ldots$ & $\ldots \ldots$ & $\ldots \ldots$ & $\ldots \ldots$ & $\ldots \ldots$ \\
\hline $\begin{array}{r}1 \\
50\end{array}$ & $\begin{array}{l}3410.03 \\
3410.742\end{array}$ & $\begin{array}{l}29316.85 \\
29310.73\end{array}$ & $\begin{array}{l}a{ }^{2} \mathrm{I}_{512}-z^{2} \mathrm{I}_{61 / 2}^{\circ} \\
b^{2}{ }^{2} \mathrm{G}_{413}-y^{4} \mathrm{~F}_{411}^{0}\end{array}$ & $\overline{6}^{---}$ & $(0.08)$ & 1.07 & 1.15 & $.35-$ & $1.11+$ \\
\hline 10 & 3411.43 & 29304.8 & $a^{2} \mathrm{~F}_{21 / 2}-z{ }^{6} \mathrm{~F}_{31 / 2}^{11 / 2}$ & -...- & $(0.00)$ & 1.01 & $\begin{array}{c}1.10 \\
--.-1\end{array}$ & $\begin{array}{c}.00- \\
-\ldots--.\end{array}$ & $1.11+$ \\
\hline 5 & 3416.612 & 29260.37 & $b^{2} \mathrm{G}_{31 / 2}-z^{2} \mathrm{G}_{41 / 2}^{0}$ & 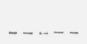 & $-\ldots---$ & $\ldots \ldots--$ & $\ldots-\cdots$ & $\ldots-\cdots$ & - - - \\
\hline 1 & 3424.018 & 29197.08 & $b^{2} \mathrm{G}_{41 / 2}^{0 / 2}-z^{2} \mathrm{G}_{31 / 2}^{10}$ & $\ldots$ & $\ldots-\ldots$ & $\ldots \ldots$ & $\ldots \ldots$ & $\ldots \ldots$ & $\ldots \ldots$ \\
\hline 1 & 3424. 131 & 29196. 12 & $b{ }^{2} \mathrm{D}_{21 / 2}-z{ }^{4} \mathrm{G}_{21 / 2}$ & $\ldots$ & $\ldots \ldots$ & $\ldots-\ldots$ & $\ldots \ldots$ & $\ldots \ldots$ & $\ldots \ldots$ \\
\hline $\begin{array}{l}5 \\
6\end{array}$ & $\begin{array}{l}3424.381 \\
3427.728\end{array}$ & $\begin{array}{l}29193.99 \\
29165.48\end{array}$ & $b^{4} \mathrm{D}_{31 / 2}-z{ }^{4} \mathrm{H}_{31 / 2}^{8}$ & 6 & $(0-06)$ & & 100 & $-\cdots$ & - - n \\
\hline 0 & 5427.120 & 29100.40 & $0-\mathrm{F}_{21 / 2}-y=\mathrm{F}_{21 / 2}$ & & $(0.00)$ & $(0.945)$ & 1.00 & .10 & \\
\hline 1 & 3433.10 & 29119. 84 & & & $\ldots-\cdots$ & $-\ldots-\ldots$ & $\ldots-\cdots$ & $\ldots \ldots$ & --- \\
\hline 2 & 3434.02 & 29112. 04 & $b^{2} \mathrm{D}_{21 / 2}-z{ }^{4} \mathrm{G}_{31 / 2}^{\circ}$ & $-\frac{-}{5}$ & 0.339 & 0954 & -703 & $-\cdots-\cdots$ & - \\
\hline $\begin{array}{l}10 \\
10\end{array}$ & $\begin{array}{l}3446.750 \\
3462983\end{array}$ & $\begin{array}{l}29004.53 \\
28868.57\end{array}$ & 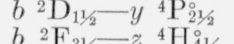 & $\begin{array}{l}5 \\
4\end{array}$ & $\begin{array}{r}0.339 \\
(0.04)\end{array}$ & $\begin{array}{l}0.954 \\
1.04\end{array}$ & $\begin{array}{l}\text { 1. } 293 \\
\text { 1. } 00\end{array}$ & .170 & 1. 801 \\
\hline 10 & 3465.405 & 28848. 39 & $d^{2} \mathbf{D}_{11 / 2}-y^{2}{ }^{2} \mathbf{F}_{21 / 2}^{11 / 2}$ & $-\ldots$ & $(0.0 x)$ & $\begin{array}{l}1.0 x \\
-\ldots-1--\end{array}$ & 1.00 & $.15 w$ & $0.80+$ \\
\hline 5 & 3469.269 & 28816.26 & $c^{2} \mathrm{G}_{41 / 2}-z^{2} \mathrm{I}_{51 / 2}^{\circ}$ & $\ldots$ & 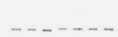 & $\ldots$ & & & \\
\hline 1 & 3469.907 & 28810.97 & $b^{2} \mathrm{G}_{31 / 2}-y{ }^{4} \mathbf{F}_{41 / 2}^{2}$ & - . . - & - n- & - - - - - & $\ldots . .$. & $\begin{array}{ll}--1 \\
-n-1\end{array}$ & - \\
\hline 1 & 3473.195 & 28783.69 & b ${ }^{4} \mathrm{P}_{21 / 2}-z{ }^{4} \mathrm{P}_{21 / 2}^{1}$ & & $\ldots-\ldots$ & & & 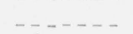 & \\
\hline 5 & 3479. 339 & 28732.87 & $b^{2}{ }^{2} \mathrm{G}_{41 / 2}-y{ }^{4} \mathrm{~F}_{31 / 2}^{0}$ & 7 & - & (1.067) & 1.03 & 0 & 1. 20 \\
\hline 2 & 3480.060 & 28720.91 & $b{ }^{2} \mathrm{~F}_{21 / 2}-z{ }^{4} \mathrm{G}_{21 / 2}$ & 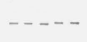 & $\ldots \ldots$ & $\ldots \ldots$ & $-\ldots \ldots$ & $\ldots \ldots-$ & $-\ldots \ldots$ \\
\hline 2 & 3481.928 & 28711.50 & $b{ }^{2} \mathrm{P}_{11 / 2}-z{ }^{4} \mathrm{~S}_{11 / 2}^{0}$ & $\ldots$ & 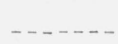 & $\ldots \ldots$ & $\ldots-\cdots$ & $\ldots \ldots-$ & $-\ldots-\ldots-$ \\
\hline 5 & 3483. 649 & 28697. 32 & $b^{2}{ }^{2} \mathrm{G}_{31 / 2}-z^{2} \mathrm{G}_{31 / 2}^{0}$ & - . - & - . - - & $-\ldots \ldots$ & $\ldots \ldots$ & $\ldots \ldots$ & - n \\
\hline 5 & 3487.672 & 28664.22 & $c^{2} \mathrm{D}_{21 / 2}-y{ }^{4} \mathrm{G}_{31 / 2}^{11}$ & $\ldots--$ & $\ldots \ldots-$ & ----- & $\ldots \ldots--$ & ----- & $\ldots \ldots \ldots$ \\
\hline 5 & 3490. 277 & 28642.82 & $b{ }^{2} \mathrm{~F}_{21 / 2}-z{ }^{4} \mathrm{G}_{31 / 2}^{0}$ & $-\ldots$ & ----- & $\ldots \ldots$ & 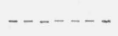 & $\ldots \ldots-$ & $\ldots \ldots$ \\
\hline 10 & 3500.86 & 28556. 24 & $a{ }^{2} \mathrm{~F}_{31 / 2}-z{ }^{6} \mathrm{~F}_{41 / 2}^{0}$ & $-\cdots$ & 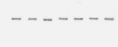 & $\ldots \ldots$ & $\ldots \ldots-\cdots$ & $\ldots \ldots$ & $-\ldots-\cdots$ \\
\hline 2 & 3504.56 & 28526. 09 & $b{ }^{4} \mathrm{~F}_{21 / 2}-z{ }^{4} \mathrm{P}_{11 / 2}^{0}$ & & & & & & \\
\hline 200 & 3509. 217 & 28488. 24 & $b^{2} \mathrm{~F}_{31 / 2}-z{ }^{4} \mathrm{H}_{31 / 2}$ & 6 & 0.121 & 1. 082 & 0.961 & 0.427 & 1. 021 \\
\hline 2 & 3511.40 & 28470.53 & & $-\ldots$ & ------ & $--\ldots-n$ & $\ldots \ldots-\cdots$ & $\ldots \ldots \ldots$ & $\ldots \ldots-\ldots$ \\
\hline 20 & 3520.063 & 28400.46 & $d^{2} \mathrm{D}_{21 / 2}-y^{2} \mathrm{~F}_{31 / 2}^{\circ}$ & & $---\frac{1}{10}-$ & & $\cdots+\cdots$ & $\cdots-\frac{-1}{2}$ & $-\cdots-\cdots$ \\
\hline 6 & 3523. 917 & 28369. 40 & $b^{2} \mathrm{G}_{41 / 2}-z^{4} \mathrm{I}_{41 / 2}^{0}$ & 6 & .12 & (1.067) & .95 & $.54-$ & $?$ \\
\hline 20 & 3524.480 & 28364. 87 & $d^{2} \mathrm{D}_{11 / 2}-x{ }^{4} \mathrm{P}_{21 / 2}^{0}$ & - - & $-\ldots-n$ & -..- & 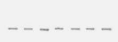 & $\ldots \ldots$ & $-\ldots \ldots$ \\
\hline 20 & 3527. 183 & 28343. 13 & $c^{2} \mathrm{G}_{41 / 2}=z^{2} \mathrm{G}_{41 / 2}$ & $-\ldots$ & $\ldots----$ & $\ldots \ldots-$. & $\ldots \ldots-$ & $\ldots \ldots \ldots$ & $\ldots \ldots \ldots$ \\
\hline 2 & 3542.617 & 28219. 65 & $a{ }^{2} \mathrm{~S}_{01 / 2}-z{ }^{2} \mathrm{P}_{11 / 2}^{0}$ & $-\ldots$ & 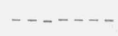 & $\ldots \ldots-\ldots$ & $\ldots \ldots \ldots$ & $\ldots \ldots-\ldots$ & - - . . . \\
\hline 10 & 3548.508 & 28172. 81 & $b \quad{ }^{4} \mathrm{P}_{11 / 2}-z{ }^{4} \mathrm{P}_{01 / 2}^{0}$ & ---- & $\ldots \ldots-\cdots$ & $-\ldots-\cdots$ & $\cdots-\cdots$ & $\ldots-\cdots-$ & $-\ldots-\ldots-$ \\
\hline 10 & 3556.816 & 28107. 01 & $a^{4} \mathrm{H}_{41 / 2}-z{ }^{4} \mathrm{~F}_{31 / 2}^{0}$ & $-\cdots-$ & $-\cdots--$ & 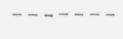 & $-\cdots-\cdots$ & 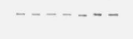 & 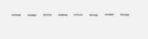 \\
\hline 5 & 3565.437 & 28039. 05 & $c^{2} \mathrm{G}_{41 / 2}-z{ }^{4} \mathrm{I}_{51 / 2}^{\circ}$ & & $\cdots-\cdots$ & -- & 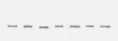 & $\ldots$ & $\ldots \ldots$ \\
\hline 10 & 3566.452 & 28031.07 & $c^{2} \mathrm{G}_{31 / 2}-z^{2} \mathrm{~F}_{31 / 2}^{1 / 2}$ & & $\ldots-\ldots$ & $--\ldots--$ & $-\ldots-\ldots$ & $\ldots \ldots-$ & $\ldots \ldots$ \\
\hline 6 & 3577.045 & 27948. 06 & $b^{2} \mathrm{G}_{31 / 2}-y{ }^{4} \mathrm{~F}_{21 / 2}^{0}$ & $-\ldots$ & $\ldots \ldots$ & $-\ldots-\cdots$ & - n. & $-\cdots-\ldots$ & $\ldots-\ldots-n$ \\
\hline 1 & 3582. 395 & 27906. 32 & $a^{4} \mathrm{D}_{31 / 2}-z{ }^{6} \mathrm{D}_{21 / 2}^{\circ}$ & & & & $---1-\cdots$ & $-\ldots--\cdots$ & \\
\hline 20 & 3584.022 & 27893.65 & $c^{2} \mathrm{G}_{41 / 2}-y{ }^{4} \mathrm{~F}_{41 / 2}^{\circ}$ & 6 & $(0.07)$ & 1. 11 & 1. 18 & $.31-$ & 1. $15 \pm$ \\
\hline 4 & 3585.43 & 27882. 70 & $b^{4} \mathrm{P}_{21 / 2}-z{ }^{4} \mathrm{~F}_{21 / 2}^{0}$ & & & & & & \\
\hline 30 & 3591.518 & 27835.44 & $b^{2} \mathrm{G}_{41 / 2}-z^{4} \mathrm{G}_{41 / 2}^{0}$ & 6 & $(0.05)$ & 1. 05 & 1. 10 & $.24-$ & $1.07 \pm$ \\
\hline 2 & 3601.613 & 27757.42 & $a^{4} \mathrm{H}_{31 / 2}-z^{4} \mathrm{~F}_{31 / 2}^{0}$ & - . & $\ldots-\ldots$ & - - - - - & _. $\ldots \ldots$ & $\ldots \ldots$ & $\ldots-\ldots \ldots$ \\
\hline 2 & 3603. 059 & 27746.28 & $b{ }^{4} \mathrm{~F}_{31 / 2}-z{ }^{4} \mathrm{P}_{21 / 2}^{0}$ & $-\ldots$ & $-\ldots--$ & ----- & $\ldots \ldots$ & $\ldots \ldots$ & - n- \\
\hline 5 & 3612.482 & 27673. 91 & $a{ }^{2} \mathrm{~S}_{01 / 2}-y{ }^{4} \mathrm{D}_{11 / 2}^{\circ}$ & $-\ldots$ & $\ldots \ldots$ & $-\ldots-\cdots$ & $\ldots \ldots$ & $\ldots \ldots-$ & $\ldots \ldots$ \\
\hline 2 & 3615. 206 & 27653.05 & $b{ }^{2} \mathrm{D}_{11 / 2}-z{ }^{4} \mathrm{~S}_{11 / 2}$ & & $--\ldots--$ & -- & 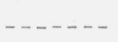 & $\ldots-\ldots$ & $\ldots \ldots$ \\
\hline 2 & 3627.896 & 27556. 33 & $b{ }^{4} \mathrm{~F}_{21 / 2}-z{ }^{4} \mathrm{P}_{21 / 2}^{0}$ &.- & ----- & ----- & 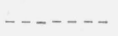 & 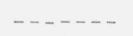 & $-\ldots-n$ \\
\hline 4 & 3628. 629 & 27550.76 & $\ldots-\cdots-\ldots$ & -.... & - n & - . . - n & $\ldots \ldots$ & $\ldots \ldots$ & $\ldots \ldots$ \\
\hline 2 & 3645. 201 & 27425.51 & $b^{2} \mathrm{G}_{31 / 2}-z^{4} \mathrm{G}_{31 / 2}$ & 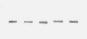 & $\ldots-\ldots$ & 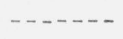 & $\cdots-\cdots$ & $\ldots-\cdots$ & 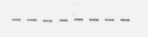 \\
\hline 2 & 3649. 771 & 27391. 18 & $c{ }^{2} \mathrm{D}_{11 / 2}-x{ }^{4} \mathrm{D}_{11 / 2}^{\circ}$ & & ----- & ------ & 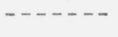 & 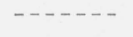 & $-\cdots-1$ \\
\hline 5 & 3652.836 & 27368. 19 & $a \quad{ }^{2} \mathrm{I}_{61 / 2}-z \quad{ }^{4} \mathrm{H}_{51 / 2}^{\circ}$ & & & & & & \\
\hline 60 & 3657.571 & 27332. 76 & $a^{4} \mathrm{D}_{31 / 2}-z^{6} \mathrm{D}_{31 / 2}^{\circ}$ & 6 & 0.165 & 1. 403 & 1. 568 & 0.579 & 1. 486 \\
\hline 30 & 3660. 070 & 27314. 10 & $b{ }^{4} \mathrm{P}_{21 / 2}-z{ }^{4} \mathrm{~F}_{31 / 2}^{0}$ & 4 & .296 & 1. 581 & 1. 285 & .149 & $0.545 ?$ \\
\hline 1 & 3666. $07 ?$ & 27291. 73 & $b^{2} \mathrm{G}_{41 / 2}-z{ }^{4} \mathrm{H}_{51 / 2}^{0}$ & $-\ldots$ & ----- & ------ & $\ldots-\ldots$ & $\ldots \ldots$ & $-\ldots-\ldots$ \\
\hline 2 & 3666.88 & 27263.38 & $c^{2} \mathrm{G}_{31 / 2}-z^{2} \mathrm{D}_{21 / 2}^{\circ}$ & & $-\ldots-.-$ & $\ldots-\cdots$ & $-\cdots-$ & 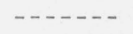 & $-\ldots-n$ \\
\hline
\end{tabular}


TABLE 6. The second spectrum of ruthenium (Ru II) - Continued

\begin{tabular}{|c|c|c|c|c|c|c|c|c|c|}
\hline $\begin{array}{c}1 \\
\text { Intensity }\end{array}$ & $\begin{array}{c}2 \\
\lambda(\text { air })\end{array}$ & $\begin{array}{l}3 \\
\sigma\end{array}$ & $\begin{array}{c}4 \\
\text { Term combina- } \\
\text { tion }\end{array}$ & $\begin{array}{c}5 \\
\text { Type }\end{array}$ & $\begin{array}{c}6 \\
\Delta g\end{array}$ & 7 & $\begin{array}{l}8 \\
g\end{array}$ & $\begin{array}{c}9 \\
\text { Strong } p\end{array}$ & $\begin{array}{c}10 \\
\text { Strong } n\end{array}$ \\
\hline & $A$ & $K$ & & & & & & & \\
\hline 2 & 3675.603 & 27198. 67 & $a^{4} \mathrm{H}_{41 / 2}-z^{4} \mathrm{D}_{31 / 2}^{0}$ & $-\cdots$ & - - - & $\ldots-\cdots$ & - - - & 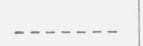 & $\ldots \ldots$ \\
\hline $\begin{array}{l}4 \\
2\end{array}$ & 3676. 289 & 27193. 60 & $a^{4} \mathrm{D}_{21 / 2}-z{ }^{6} \mathrm{D}_{11 / 2}$ & & $---\cdots$ & - n... & $-\ldots-\ldots$ & 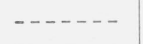 & \\
\hline $\begin{array}{r}2 \\
150\end{array}$ & $\begin{array}{l}3681.658 \\
3690.030\end{array}$ & $\begin{array}{l}27153.95 \\
27092.34\end{array}$ & 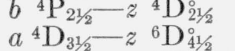 & $-\overline{5}^{---}$ & .129 & 1.401 & 1.530 & .065 & 1. 981 \\
\hline 10 & 3690.995 & 27085. 26 & $b^{4} \mathrm{~F}_{21 / 2}^{3 / 2} z{ }^{4} \mathrm{~F}_{1 / 2}^{41 / 2}$ & 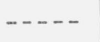 & $\ldots \ldots$ & $\ldots \ldots$ & $\ldots \ldots$ & $\ldots \ldots$ & $-\ldots-1-$ \\
\hline 6 & 3707.976 & 26961. 22 & $d^{2} \mathrm{D}_{21 / 2}-y{ }^{2} \mathrm{D}_{21 / 2}^{\circ}$ & & & & & & \\
\hline 10 & 3709.21 & 26952. 25 & $c^{2} \mathrm{G}_{41 / 2}-z^{4} \mathrm{I}_{41 / 2}^{21 / 2}$ & 6 & .13 & $(1.078)$ & 0.95 & .59 & $?$ \\
\hline 2 & 3710.998 & 26939. 27 & $c{ }^{2} \mathrm{D}_{11 / 2}-x{ }^{4} \mathrm{D}_{21 / 2}$ & ---- & $\ldots \ldots$ & $\ldots \ldots$ & $\ldots \ldots$ & $\ldots \ldots$ & $\ldots+\ldots$ \\
\hline 2 & 3713. 432 & 26921. 61 & c ${ }^{2} \mathrm{D}_{11 / 2}-y{ }^{4} \mathrm{D}_{01 / 2}^{0}$ & $-\cdots-$ & - - - - & 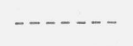 & $\ldots \ldots$ & $\ldots \ldots$ & $\ldots \ldots$ \\
\hline 2 & 3720.627 & 26869.55 & $d^{2} \mathrm{D}_{11 / 2}-x{ }^{4} \mathrm{~F}_{11 / 2}^{\circ}$ & ---- & $-\cdots--$ & $\cdots-\cdots$ & $\ldots-\cdots$ & $\ldots-\cdots$ & 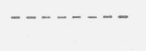 \\
\hline 5 & 3722.645 & 26854. 98 & $a{ }^{4} \mathrm{H}_{51 / 2}-z{ }^{4} \mathrm{~F}_{41 / 2}^{\circ}$ & & & & & $-\cdots-\bar{r}-$ & \\
\hline 50 & $\begin{array}{l}3734.455 \\
3734834\end{array}$ & 26770. 06 & $a^{4} \mathrm{D}_{21 / 2}-z{ }^{6} \mathrm{D}_{21 / 2}^{\circ}$ & 6 & .296 & 1. 338 & 1. 634 & .750 & 1. 486 \\
\hline $\begin{array}{l}4 \\
5\end{array}$ & $\begin{array}{l}3734.834 \\
3740.230\end{array}$ & $\begin{array}{l}26767.34 \\
26728.73\end{array}$ & 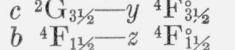 & --- & $-\cdots-1$ & $-\cdots-1$ & $-\cdots-1$ & $\ldots-\cdots$ & $-\cdots---$ \\
\hline 10 & 3746.932 & 26680. 92 & $b \quad{ }^{4} \mathbf{F}_{41 / 2}^{1 / 2} z{ }^{4} \mathrm{~F}_{31 / 2}^{1 / 2}$ & $\begin{array}{ll}-1-1 \\
-1\end{array}$ & $-1-1-1$ & $-1-1-1$ & - n-n & 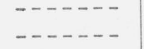 & n-n \\
\hline 5 & $3747.453 ?$ & 26677. 21 & $a^{2} \mathrm{I}_{51 / 2}-z{ }^{4} \mathrm{H}_{41 / 2}^{\circ}$ & & & & & & \\
\hline 8 & 3750.508 & 26655. 48 & $b^{4} \mathrm{~F}_{21 / 2}-z{ }^{4} \mathrm{~F}_{21 / 2}^{0}$ & 6 & .134 & 1. 083 & 0.949 & .344 & 1. 016 \\
\hline 2 & 3757. 730 & 26604. 25 & $b{ }^{4} \mathrm{P}_{11 / 2}-z{ }^{4} \mathrm{P}_{21 / 2}$ & $-\cdots-$ & $-\ldots-\cdots$ & $-\cdots--$ & $\ldots-\ldots$ & $\ldots \ldots$ & $\ldots \ldots$ \\
\hline $\begin{array}{r}1 \\
10\end{array}$ & & $\begin{array}{l}26540.76 \\
26524.62\end{array}$ & $d^{2} \mathrm{D}_{21 / 2}-1_{11 / 2}$ & $-\cdots$ & $-\cdots \overline{2}$ & & & $\ldots-\ldots--$ & \\
\hline 10 & & 26524.62 & $b^{4} \mathrm{~F}_{21 / 2}-z{ }^{4} \mathrm{D}_{11 / 2}^{\circ}$ & 4 & .207 & 0.943 & 1. 151 & .103 & 0.634 \\
\hline 10 & 3774. 474 & 26486. 23 & $b^{4} \mathrm{~F}_{11 / 2}-z^{4} \mathrm{D}_{01 / 2}^{0}$ & 5 & .444 & .422 & 0.022 & .222 & .644 \\
\hline 6 & 3777.923 & 26462. 05 & $a^{4} \mathrm{D}_{11 / 2}-z{ }^{6} \mathrm{D}_{11 / 2}{ }^{\circ}$ & 6 & 0.652 & 1. 189 & 1. 841 & 0.978 & 1. 515 \\
\hline 10 & 3784.175 & 26418. 34 & $c^{2} \mathrm{G}_{41 / 2}-z{ }^{4} \mathrm{G}_{41 / 2}$ & 6 & $(0.021)$ & (1.078) & 1. 099 & .094 & \\
\hline 4 & 3795. 691 & 26338. 19 & $a^{4} \mathrm{D}_{01 / 2}-z{ }^{6} \mathrm{D}_{01 / 2}^{\circ}$ & -- & $--\overline{102}$ & $(1-002)$ & $-\overline{1}-\overline{0}-$ & 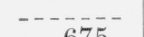 & $\cdots-\cdots$ \\
\hline 10 & 3804.572 & 26276.71 & $b{ }^{4} \mathbf{F}_{31 / 2}-z{ }^{4} \mathbf{F}_{31 / 2}^{\circ}$ & 6 & 0.193 & (1.093) & 1. 286 & .675 & ? \\
\hline 5 & 3806.567 & 26262. 93 & $c^{2} \mathrm{D}_{21 / 2}-z^{2} \mathrm{D}_{21 / 2}^{\circ}$ & $\ldots-$ & $\ldots \ldots$ & 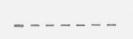 & $\ldots \ldots$ & 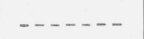 & $\ldots-\ldots$ \\
\hline 4 & 3811. 166 & 26231. 25 & $c^{2} \mathrm{D}_{21 / 2}-z^{2} \mathrm{G}_{31 / 2}^{\circ}$ & $-\cdots-$ & $-\cdots-\cdots$ & $\ldots-\cdots$ & $-\cdots--$ & $--\cdots--$ & $\ldots \ldots$ \\
\hline 2 & $\begin{array}{l}3811.805 \\
3816.207\end{array}$ & $\begin{array}{l}26226.85 \\
26196.60\end{array}$ & $a^{4} \mathrm{D}_{21 / 2}-z^{6} \mathrm{D}_{31 / 2}^{\circ}$ & $-\overline{5}^{---}$ & .233 & $(1.337)$ & 1.570 & .118 & $?$ \\
\hline 5 & 3820.386 & 26167. 94 & $b^{4} \mathrm{~F}_{11 / 2}-z^{4} \mathrm{D}_{11 / 2}$ & $\ldots$ & - . - & $-\ldots-1$ & $\ldots \ldots$ & $\ldots \ldots$ & $\ldots \ldots$ \\
\hline 3 & 3827.92 & 26116. 44 & $b^{4} \mathrm{~F}_{31 / 2}-z^{4} \mathrm{D}_{21 / 2}^{\circ}$ & 4 & .255 & (1. 093) & 1. 348 & .126 & ? \\
\hline 5 & 3832. 272 & 26086. 78 & $b^{4} \mathrm{~F}_{21 / 2}-z{ }^{4} \mathrm{~F}_{31 / 2}^{0}$ & $\ldots .$. & $\ldots \ldots$ & $\ldots \ldots$ & $\ldots \ldots$ & $\ldots \ldots$ & $\ldots$ \\
\hline $\begin{array}{l}6 \\
1\end{array}$ & $\begin{array}{l}3843.462 \\
3863.68\end{array}$ & $\begin{array}{l}26010.83 \\
25874.73\end{array}$ & $b^{2} \mathrm{G}_{31 / 2}-z{ }^{4} \mathrm{H}_{31 / 2}^{\circ}$ & & $\ldots-\cdots$ & $\ldots-\ldots$ & $\ldots \ldots$ & $-\cdots--$ & $-\ldots-\cdots$ \\
\hline 50 & 3879. 023 & 25772. 38 & $b^{4} \mathrm{H}_{41 / 2}-z^{4} \mathrm{D}_{31 / 2}^{0}$ & $\overline{4}^{-}$ & 186 & 1. 208 & 1. 394 & 0.92 & $0 . \overline{5} \overline{7}$ \\
\hline 2 & 3885.823 & 25727. 28 & $a^{2} \mathrm{~F}_{21 / 2}-z^{6} \mathrm{D}_{21 / 2}^{0}$ & $-\ldots-$ & $\ldots \ldots$ & $\ldots \ldots \ldots$ & $\ldots \ldots-$ & $\ldots \ldots$ & -....... \\
\hline 1 & 3889. 43 & 25703. 42 & b ${ }^{4} \mathrm{P}_{11 / 2}-z{ }^{4} \mathrm{~F}_{21 / 2}^{0}$ & $\ldots-$ & $---1-$ & $--1--$ & $-\ldots-1$ & $\ldots \ldots$ & - n- \\
\hline 10 & 3932. 828 & 25419. 80 & $b^{4} \mathrm{~F}_{41 / 2}-z^{4} \mathrm{~F}_{41 / 2}^{0}$ & ---- & $---ロ ニ-$ & $----\overline{-}$ & & $-\cdots---$ & \\
\hline $\begin{array}{r}60 \\
100\end{array}$ & $\begin{array}{l}4002.957 \\
4009.928\end{array}$ & 24974. 47 & $b{ }^{4} \mathrm{P}_{11 / 2}-z{ }^{4} \mathrm{D}_{21 / 2}^{0}$ & $\frac{4}{5}$ & .295 & 1. 645 & 1. 350 & .148 & .907 \\
\hline 100 & 4009.928 & 24931.06 & $a^{4} \mathrm{G}_{21 / 2}-z^{4} \mathrm{~F}_{1 / 2}^{1}$ & 5 & .186 & 0.654 & 0.468 & .093 & .933 \\
\hline
\end{tabular}

\begin{tabular}{|c|c|c|c|c|c|c|c|}
\hline \multicolumn{4}{|c|}{ Observed at pole of arc } & \multicolumn{4}{|c|}{ Observed at pole of arc } \\
\hline Intensity & $\lambda$ (vacuum) & $\sigma$ & Term combination & Intensity & $\lambda$ (vacuum) & $\sigma$ & Term combination \\
\hline $\begin{array}{r}3 \\
2 \\
5 \\
10 \\
15\end{array}$ & $\begin{array}{l}4011.30 \\
4016.96 \\
4025.28 \\
4040.25 \\
4041.56\end{array}$ & $\begin{array}{l}24922.6 \\
24887.4 \\
24836.0 \\
24744.0 \\
24736.0\end{array}$ & $\begin{array}{l}d^{2} \mathrm{D}_{21 / 2}-z^{2} \mathrm{P}_{11 / 2}^{1} \\
a^{4} \mathrm{H}_{41 / 2}-z^{6} \mathrm{~F}_{31 / 2}^{0} \\
a^{4} \mathrm{H}_{51 / 2}-z^{6} \mathrm{~F}_{41 / 2}^{3}\end{array}$ & $\begin{array}{r}2 \\
20 \\
5 \\
10 \\
10\end{array}$ & $\begin{array}{l}4093.41 \\
4104.80 \\
4134.10 \\
4135.466 \\
4143.74\end{array}$ & $\begin{array}{l}24422.3 \\
24354.9 \\
24182.3 \\
24174.3 \\
24126.0\end{array}$ & $\begin{array}{c}a^{2} \mathrm{~F}_{31 / 2}-z^{6} \mathrm{D}_{31 / 2}^{\circ} \\
a^{4} \mathrm{G}_{31 / 2}-z^{4} \mathrm{~F}_{21 / 2}^{\circ} \\
a^{2} \mathrm{~F}_{31 / 2}-z^{6} \mathrm{D}_{41 / 2}^{\circ} \\
b^{4} \mathrm{P}_{01 / 2}-z^{4} \mathrm{D}_{11 / 2}^{\circ} \\
a^{4} \mathrm{G}_{41 / 2}-z^{4} \mathrm{~F}_{31 / 2}^{0}\end{array}$ \\
\hline $\begin{array}{r}2 \\
20 \\
1 \\
2 \\
1\end{array}$ & $\begin{array}{l}4041.72 \\
4043.04 \\
4045.55 \\
4064.81 \\
4081.72\end{array}$ & $\begin{array}{l}24735.0 \\
24726.9 \\
24711.6 \\
24594.5 \\
24492.6\end{array}$ & $\begin{array}{c}b^{4} \mathrm{P}_{01 / 2}-z^{4} \mathrm{~F}_{11 / 2}^{0} \\
a^{4} \mathrm{H}_{41 / 2}-z^{6} \mathrm{~F}_{41 / 2}^{\circ} \\
a^{4} \mathrm{H}_{31 / 2}-z^{6} \mathrm{~F}_{21 / 2}^{\circ} \\
b^{4} \mathrm{P}_{11 / 2}-z^{6} \mathrm{P}_{11 / 2}^{0} \\
b^{4} \mathrm{P}_{01 / 2}-z^{4} \mathrm{D}_{01 / 2}^{\circ}\end{array}$ & $\begin{array}{r}1 \\
10 \\
20 \\
3 \\
3\end{array}$ & $\begin{array}{l}4164.76 \\
4173.991 \\
4174.912 \\
4191.95 \\
4204.62\end{array}$ & $\begin{array}{l}24004.2 \\
23951.13 \\
23945.85 \\
23848.6 \\
23776.7\end{array}$ & $\begin{array}{c}b^{4} \mathrm{~F}_{41 / 2}-z^{6} \mathrm{P}_{31 / 2}^{o} \\
b^{4} \mathrm{P}_{21 / 2}-z^{6} \mathrm{~F}_{31 / 2}^{\circ} \\
a^{4} \mathrm{G}_{51 / 2}-z^{4} \mathrm{~F}_{41 / 2}^{\circ} \\
b^{4} \mathrm{D}_{11 / 2}-z^{4} \mathrm{P}_{11 / 2}^{0}\end{array}$ \\
\hline
\end{tabular}


TABLE 6. The second spectrum of ruthenium (Ru II) - Continued

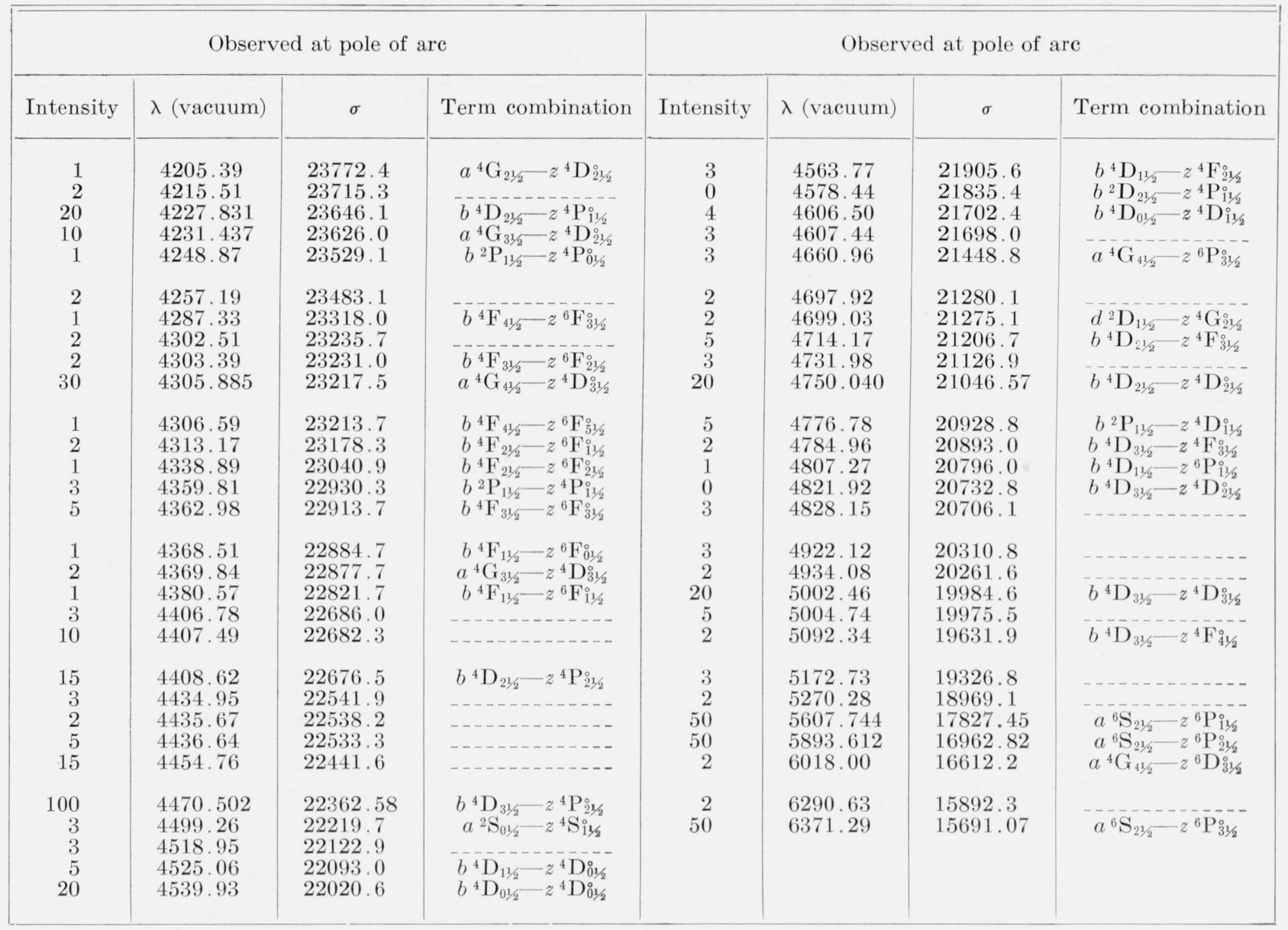

Washington, D. C. July 22, 1958. 